\title{
Comparing Zwitterionic and PEG Exteriors of Polyelectrolyte Complex Micelles
}

Jeffrey Ting, Alexander Marras, Joseph Mitchell, Trinity Campagna, Matthew Tirrell

Submitted date: 03/05/2020 - Posted date: 06/05/2020

Licence: CC BY-NC-ND 4.0

Citation information: Ting, Jeffrey; Marras, Alexander; Mitchell, Joseph; Campagna, Trinity; Tirrell, Matthew (2020): Comparing Zwitterionic and PEG Exteriors of Polyelectrolyte Complex Micelles. ChemRxiv. Preprint. https://doi.org/10.26434/chemrxiv.12237020.v1

A series of model polyelectrolyte complex micelles (PCMs) was prepared to investigate the consequences of neutral and zwitterionic chemistries and distinct charged cores on the size and stability of nanocarriers. Using aqueous reversible addition-fragmentation chain transfer (RAFT) polymerization, we synthesized a well-defined diblock polyelectrolyte system, poly(2-methacryloyloxyethyl phosphorylcholine methacrylate)-block-poly((vinylbenzyl) trimethylammonium) (PMPC-PVBTMA), at various neutral and charged block lengths to compare directly against PCM structure-property relationships centered on poly(ethylene glycol)-block-poly((vinylbenzyl) trimethylammonium) (PEG-PVBTMA) and poly(ethylene glycol)-block-poly(lysine) (PEG-PLK). After complexation with a common polyanion, poly(sodium acrylate), the resulting PCMs were characterized by dynamic light scattering (DLS) and small angle X-ray scattering (SAXS). We observed uniform assemblies of spherical micelles with a core diameter of $\sim 40 \mathrm{~nm}$ when PMPC-PVBTMA was used, and smaller particles between 20-30 nm for PEG-PLK and PEG-PVBTMA via SAXS analysis. Additionally, PEG-PLK PCMs proved most resistant to dissolution by both monovalent and divalent salt, followed by PEG-PVBTMA then PMPC-PVBTMA. All micelle systems were serum stable in $100 \%$ fetal bovine serum over the course of $8 \mathrm{~h}$ by time-resolved DLS, demonstrating minimal interactions with serum proteins and potential as in vivo drug delivery vehicles. This thorough study of the synthesis, assembly, and characterization of zwitterionic polymers in PCMs advances the design space for charge-driven micelle assemblies.

File list (2)

draft-fin.pdf (2.36 MiB)

view on ChemRxiv • download file 


\section{Comparing Zwitterionic and PEG Exteriors of}

\section{Polyelectrolyte Complex Micelles}

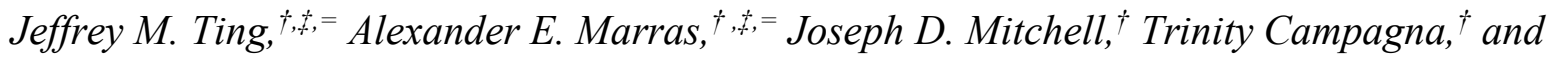

Matthew V. Tirrell ${ }^{*}+,+$,

$\dagger$ Pritzker School of Molecular Engineering, University of Chicago, Chicago, IL 60637, USA.

\$ Center for Molecular Engineering and Materials Science Division, Argonne National

Laboratory, Lemont, IL 60439, USA. 


\section{ABSTRACT}

A series of model polyelectrolyte complex micelles (PCMs) was prepared to investigate the consequences of neutral and zwitterionic chemistries and distinct charged cores on the size and stability of nanocarriers. Using aqueous reversible addition-fragmentation chain transfer (RAFT) polymerization, we synthesized a well-defined diblock polyelectrolyte system, poly(2methacryloyloxyethyl phosphorylcholine methacrylate)-block-poly((vinylbenzyl) trimethylammonium) (PMPC-PVBTMA), at various neutral and charged block lengths to compare directly against PCM structure-property relationships centered on poly(ethylene glycol)-blockpoly((vinylbenzyl) trimethylammonium) (PEG-PVBTMA) and poly(ethylene glycol)-blockpoly(lysine) (PEG-PLK). After complexation with a common polyanion, poly(sodium acrylate), the resulting PCMs were characterized by dynamic light scattering (DLS) and small angle X-ray scattering (SAXS). We observed uniform assemblies of spherical micelles with a core diameter of 40 nm when PMPC-PVBTMA was used, and smaller particles between 20-30 nm for PEG-PLK and PEG-PVBTMA via SAXS analysis. Additionally, PEG-PLK PCMs proved most resistant to dissolution by both monovalent and divalent salt, followed by PEG-PVBTMA then PMPCPVBTMA. All micelle systems were serum stable in 100\% fetal bovine serum over the course of $8 \mathrm{~h}$ by time-resolved DLS, demonstrating minimal interactions with serum proteins and potential as in vivo drug delivery vehicles. This thorough study of the synthesis, assembly, and characterization of zwitterionic polymers in PCMs advances the design space for charge-driven micelle assemblies. 


\section{Introduction}

Polyelectrolyte complex assemblies have the unique ability to interface with an array of biologics [1-4]. This liquid-liquid phase separation process upon mixing oppositely-charged polyelectrolyte solutions can be advantageous for partitioning biomolecules into compartmentalized domains [5-8]. When the complex domain is confined to the core of polyelectrolyte complex micelles (PCMs) with neutral-charged block polycation and/or polyanion architectures, therapeutically relevant cargo can be captured, protected by the outer hydrophilic corona, and released in response to changes in $\mathrm{pH}$ or ionic strength. In terms of polymer selection for the outer stealth layer, poly(ethylene glycol) (PEG; synonymously polyethylene oxide or PEO) is one of the most common ways to facilitate steric stabilization from aggregation and facilitate biocompatibility. PEG-containing PCMs can self-assemble into discrete nanoparticles with controlled size and morphologies at dilute polymer conditions $[9,10]$ and resemble ordered gels at higher polymer concentrations [11-14]. However, certain limitations of PEG have become identified in the past decade. For biological applications, this includes hypersensitivity after drug administration, deteriorating oxidative stability over time, and accelerated systemic clearance upon repeated dosage [15-17]. Thus, there has been substantial effort to explore PEG alternatives that can instill colloidal stability in PCM carriers in aqueous environments while mitigating some of these drawbacks [18-23].

One promising alternative includes polyampholytes, or polyelectrolytes with both cations and anions along the chain. Polyzwitterions are polyampholytes with paired cation and anion moieties per repeat unit along the electrostatically neutral chain $[24,25]$. The 2methacryloyloxyethyl phosphorylcholine (MPC) methacrylate monomer, in particular, has become transformative as a building block for polyzwitterions in emerging biomaterials and 
biodevices [26], as the phospholipid polar groups provide hydration but does not disturb the hydrogen bonding of water [27]. When polymerized to poly(2-methacryloyloxyethyl phosphorylcholine methacrylate) (PMPC), the zwitterionic phosphorylcholine moieties endow high resistance to nonspecific protein adsorption, cellular adhesion, and blood coagulation, as well as cell membrane penetration, molecular recognition, and underwater lubrication $[28,29]$. These bioinert "stealth" properties have been exploited in antifouling [30] and water treatment [31] materials, bioengineering [32], and nanomedicine applications [33]. Furthermore, the antipolyelectrolyte effect, where certain polyzwitterion chains swell (increased coil size and viscosity) with added salt [34-36], has been shown to not affect the solution conformation of PMPC [37,38]. These combined attributes have allowed PMPC to emerge as a versatile material platform for a diverse array of bioapplications.

When adapted to hydrophilic coronas in nanocarriers, several reports that have directly compared PMPC to PEG have shown enhanced delivery for siRNA polyplexes [33], bovine serum albumin protein nanoparticles [39], $\alpha$-chymotrypsin proteins [40], and papain enzyme bioconjugates [41]. These PMPC-containing block polymers are often synthesized using reversible-deactivation radical polymerization (RDRP). Lowe and McCormick provide an excellent review on the controlled synthesis of zwitterionic polymers [42], followed by more recent examples of PMPC systems in the literature prepared using atom transfer radical polymerization (ATRP) [43-46] or reversible addition-fragmentation chain transfer (RAFT) polymerization $[33,47,48]$. Thus, the use of RDRP to produce PMPC block polyelectrolytes for fundamental self-assembly investigations can lead to more efficacious PCM vehicles in terms of biomolecule storage and delivery. For example, Takahashi and coworkers have reported on how $\mathrm{NaCl}$ can trigger morphological transitions in PCM assemblies comprising RAFT PMPC block 
polyelectrolytes [49]. These results have advanced our overall understanding of how to tailor the static and dynamic properties of polyelectrolyte complex assemblies.

The objective of this work is to evaluate and compare the formulation and stability of polyelectrolyte-based nanocarriers, comprising PMPC and PEG as hydrophilic coronas and chemically distinct charged cores. Using aqueous RAFT polymerization, we have previously established a synthetic framework to prepare designer block polyelectrolytes from PEG macromolecular RAFT chain transfer agents [50]. Specifically, the diblock polycation PEG-blockpoly((vinylbenzyl)trimethylammonium chloride) (PEG-PVBTMA) has been paired with various synthetic polyanion architectures, including poly(sodium acrylate) (PAA) [50-53], PEG-blockpoly(styrene sulfonate) [50,54], and DNA oligonucleotides [55,56], to form assemblies of varying size, shape, and responsivity to salt. Because of the ease and versatility of RAFT chemistry for the production of stimuli-responsive materials [57], these endeavors have enabled us to establish structure-property relationships with special attention to effects of modulating charged block length, chemical identity, and polyelectrolyte pairing. Figure 1 depicts the extension of this molecular engineering effort by comparing PCMs of PAA with three diblock polycations: PEGblock-poly(lysine) (PEG-PLK), PEG-PVBTMA and PMPC-PVBTMA. For ease of nomenclature and distinction, we refer to PMPC-containing systems as zwitterionic PCMs (zPCMs). 


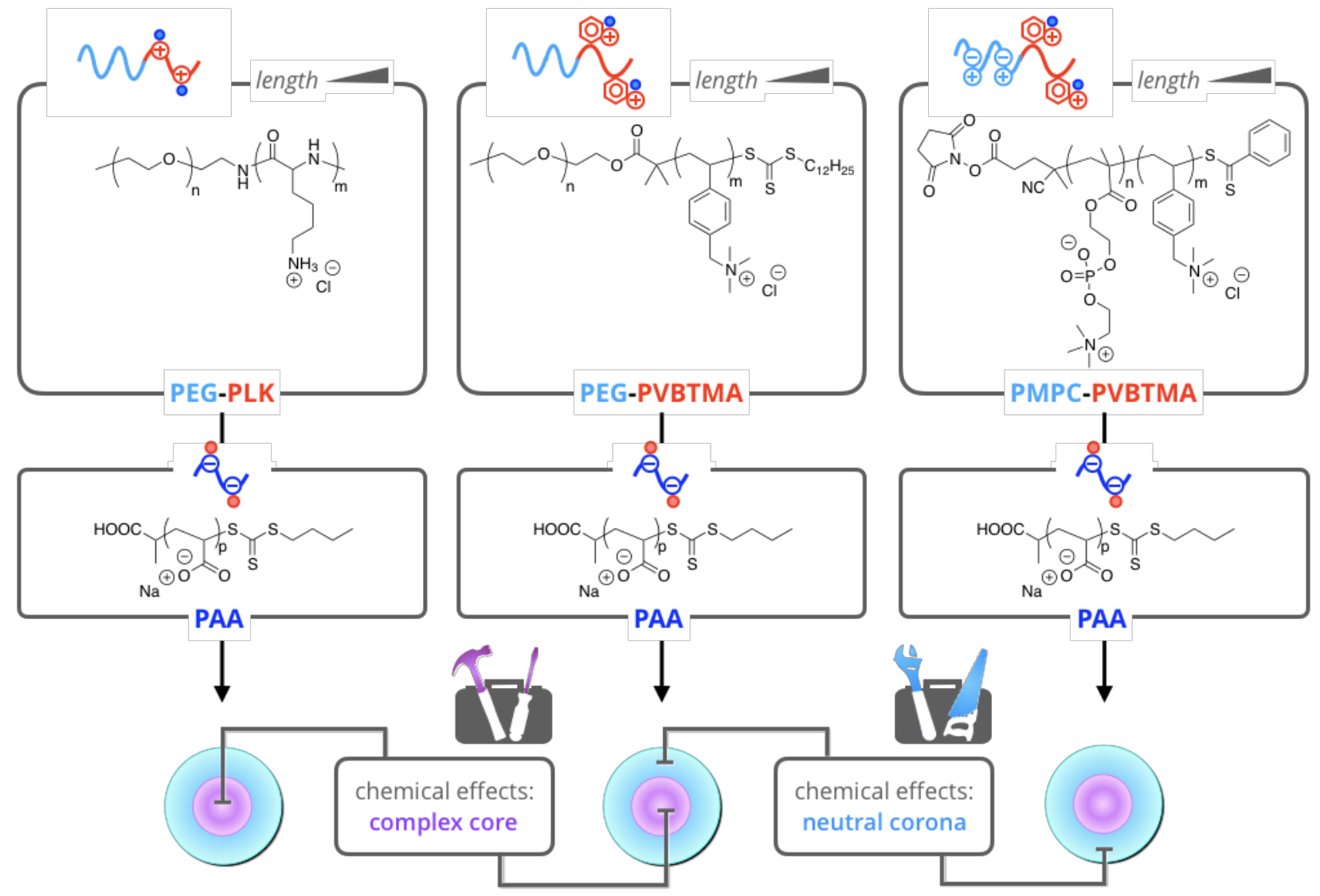

Figure 1. Chemical structures of polyelectrolytes and illustration of the rational pairings to investigate the chemical effects of the complex core and neutral corona in complex coacervate micelles. Diblock polycations include poly(ethylene glycol)-block-poly(lysine) (PEG-PLK), poly(ethylene glycol)-block-poly((vinylbenzyl)trimethylammonium chloride) (PEG-PVBTMA), and poly(2-methacryloyloxyethyl phosphorylcholine methacrylate)-blockpoly(vinylbenzyl)trimethylammonium chloride) (PMPC-PVBTMA). Poly(sodium acrylate) (PAA) was used as the polyanion for all cases. See Table 1 for polymer length and characterization. 


\section{Materials and Methods}

\subsection{Materials}

Certain commercial equipment and materials are identified in this paper in order to specify adequately the experimental procedure. In no case does such identification imply recommendations by the National Institute of Standards and Technology (NIST) nor does it imply that the material or equipment identified is necessarily the best available for this purpose.

The following chemicals were reagent grade and used as received unless otherwise specified: poly(ethylene glycol)-block-poly(lysine) (Alamanda Polymers; Huntsville, AL, USA), poly(ethylene glycol) methyl ether (2-methyl-2-propionic acid dodecyl trithiocarbonate) (Sigma, Reported $M_{\mathrm{n}} 10,000$ and $5000 \mathrm{~g} / \mathrm{mol}$; verified experimentally by ${ }^{1} \mathrm{H}$ NMR spectroscopy and SECMALS with $Ð=1.02$ and 1.01, respectively), 2-methacryloyloxyethyl phosphorylcholine (MPC, Sigma, 97\%), 4-cyano-4-(phenylcarbonothioylthio)pentanoic acid N-succinimidyl ester (CPSE, Sigma), 2-(butylthiocarbonothioylthio)propanoic acid (BuPA, Boron Molecular, $\geq 95 \%$ ), sodium acrylate (NaA, Sigma, 97\%), (vinylbenzyl)trimethylammonium chloride (VBTMA, Sigma, 99\%), 2,2'-azobis[2-(2-imidazolin-2-yl)propane]dihydrochloride (VA-044, Wako Chemicals, USA), acetic acid (glacial, Sigma, $\geq 99.85 \%$ ), sodium acetate trihydrate (Sigma, $\geq 99 \%$ ), sodium chloride (Sigma, $\geq 99.0 \%$ ), trifluoroacetic acid (TFA, ReagentPlus $\left.{ }^{\circledR}, 99 \%\right)$, sodium nitrate $\left(\mathrm{NaNO}_{3}, \mathrm{ACS}\right.$ reagent, $\geq 99.0 \%$ ), sodium azide $\left(\mathrm{NaN}_{3}\right.$, ReagentPlus ${ }^{\circledR}, \geq 99.5 \%$ ), Fetal Bovine Serum (ES CellQualified, Thermo Fisher, USA), pooled normal human AB serum (Innovative Research Inc., Novi, Michigan), and SnakeSkin dialysis tubing (MWCO 3.5K, $22 \mathrm{~mm}$, Thermo Scientific).

For RAFT polymerizations, monomers VBTMA and NaA were used as received. MPC monomer was filtered with diethyl ether to remove trace inhibitor, vacuum dried, and stored under dried nitrogen until use. Acetate buffer solution ( $\mathrm{pH}$ 5.2) was prepared from $0.1 \mathrm{M}$ acetic acid and 
$0.1 \mathrm{M}$ sodium acetate trihydrate $(42 / 158, v / v)$. Water was filtered from a Milli-Q water purification system at a resistivity of $18.2 \mathrm{M} \Omega-\mathrm{cm}$ at $25^{\circ} \mathrm{C}$.

\subsection{RAFT Synthesis of PEG-PVBTMA, PAA, and PMPC-PVBTMA}

We synthesized PEG-PVBTMA with aqueous RAFT polymerization from a PEG macromolecular chain transfer agent (macro CTA), according to parallel synthesis and characterization procedures described by Ting et al. [30] previously. PAA was synthesized using BuPA as a RAFT chain transfer agent (CTA) in aqueous settings and characterized. Detailed molecular characterization is provided in the Supplementary Materials Section S1.

Scheme 1 depicts the synthesis of PMPC and PMPC-PVBTMA. The following describes a representative RAFT protocol: To a dried round bottom flask, the chemical precursors (monomer, RAFT CTA, and VA-044 initiator) were combined in solvent and sealed. A ratio of 10:1 equivalence of RAFT CTA to initiator $\left([\mathrm{CTA}]_{0} /[\mathrm{I}]_{0}=10: 1\right)$ was used. The flask was degassed for at least $30 \mathrm{~min}$ with dried nitrogen sparging, before running the reaction at $50{ }^{\circ} \mathrm{C}$ under constant stirring for $>18 \mathrm{~h}$. The final monomer conversion was confirmed by ${ }^{1} \mathrm{H}$ NMR spectroscopy before quenching by cooling to room temperature and opening the reactor to air. The crude red-orange mixture was dialyzed against Milli-Q water for 3 cycles of $8 \mathrm{~h}$ each, lyophilized, and characterized by size-exclusion chromatography with multiangle light scattering, ${ }^{1} \mathrm{H}$ NMR spectroscopy, and thermogravimetric analysis. 
Scheme 1. Synthesis of PMPC- $b$-PVBTMA via RAFT Polymerization.

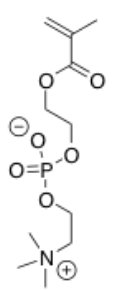

MPC

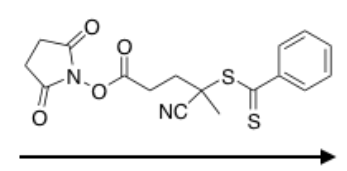

VA- $044,50^{\circ} \mathrm{C}$ Methanol

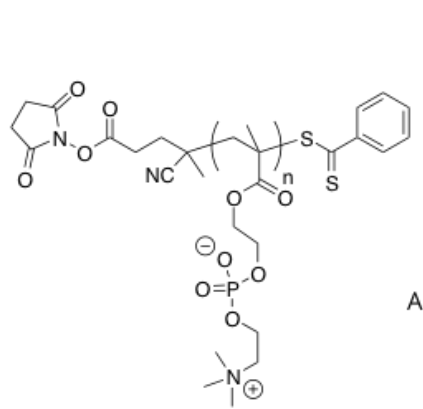

PMPC

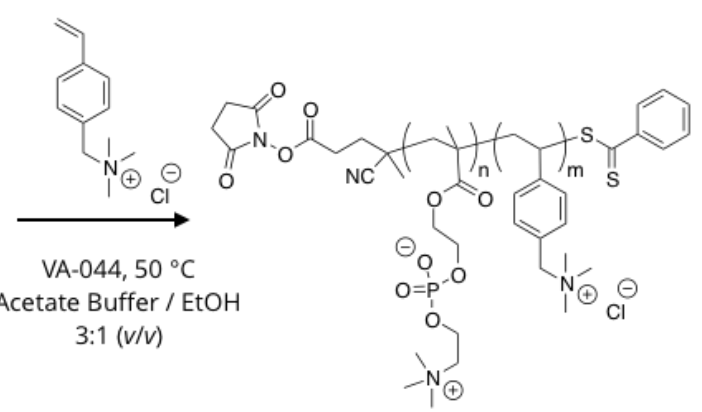

PMPC-b-PVBTMA

\subsection{Size-Exclusion Chromatography with Multiangle Light Scattering (SEC-MALS)}

SEC measurements were conducted on a Waters liquid chromatogram (well-stirred mobile phase reservoir, Waters In-Line Degasser AF, Waters 1515 Isocratic HPLC pump, Waters 2707 Autosampler) equipped with three Waters columns (Ultrahydrogel Column 500, Ultrahydrogel Column 120, and Ultrahydrogel Column 250) maintained at $35{ }^{\circ} \mathrm{C}$ with pore sizes suitable for materials with effective molecular weights from 1000 to $400,000 \mathrm{~g} / \mathrm{mol}$. The SEC is equipped with a Waters 2998 photodiode array detector, a miniDAWN TREOS II multi-angle laser light scattering (MALS) detector at a laser wavelength of $658 \mathrm{~nm}$ (3 angles), and a Wyatt Optilab TrEX refractive index detector. Empower 3 and ASTRA ${ }^{\circledR} 6$ Software were used for setting up the experiments and analyzing data, respectively.

Samples were dissolved and immediately run. For PEG and PMPC, $0.1 \mathrm{M} \mathrm{NaNO}_{3}+0.01 \%$ $\mathrm{NaN}_{3}$ was run as the mobile phase at $1.0 \mathrm{~mL} / \mathrm{min}$ at $35^{\circ} \mathrm{C}$. For PAA, $0.3 \mathrm{M} \mathrm{NaNO} 3+0.01 \mathrm{M}$ $\mathrm{NaH}_{2} \mathrm{PO}_{4}$ at $\mathrm{pH} 7$ was run as the mobile phase at $1.0 \mathrm{~mL} / \mathrm{min}$ at $25^{\circ} \mathrm{C}$. For diblock cationic polyelectrolytes, a mixture of acetonitrile and water $(40 / 60, \%, v / v)$ with $0.1 \%$ trifluoroacetic acid was run as the mobile phase at $1.0 \mathrm{~mL} / \mathrm{min}$ at $35^{\circ} \mathrm{C}$. The change in refractive index value $(\mathrm{d} n / \mathrm{dc})$ values were determined using an Abbe refractometer with a red light-emitting diode as a light 
source in the appropriate SEC mobile phase at $25^{\circ} \mathrm{C}$, in which refractive index values at various polymer concentrations were collected in triplicate (Supplementary Figure S-X). The $\mathrm{d} n / \mathrm{dc}$ of each block polyelectrolyte $(\mathrm{d} n / \mathrm{dc})_{\text {block }}$ was calculated using Equation 1, which assumes that the $(\mathrm{d} n / \mathrm{dc})_{\text {block }}$ is a weighted linear combination (i.e., the weight fraction of each block) of the change in each refractive index value of individual polymers $A$ and $B$.

$$
\left(\frac{d n}{d c}\right)_{b l o c k}=w_{A}\left(\frac{d n}{d c}\right)_{A}+\left(1-w_{A}\right)\left(\frac{d n}{d c}\right)_{B}
$$

\subsection{Proton Nuclear Magnetic Resonance $\left({ }^{1} H\right.$ NMR) Spectroscopy}

${ }^{1} \mathrm{H}$ NMR measurements were collected using a Bruker AVANCE III HD $400 \mathrm{Mhz}$ NanoBay spectrometer with 16 transients to minimize signal-to-noise. ${ }^{1} \mathrm{H}$ NMR spectra were processed and analyzed using iNMR (Version 5.5.7).

A representative ${ }^{1} \mathrm{H}$ NMR spectrum of the PMPC homopolymer is shown in Figure $2 .{ }^{1} \mathrm{H}$ NMR (400 MHz, $\left.\mathrm{D}_{2} \mathrm{O}\right) \delta:$ 0.6-1.3 ppm $\left(2 \mathrm{H},-\mathrm{CH}_{2}-\right)$, 1.6-2.2 ppm $\left(3 \mathrm{H},-\mathrm{CH}_{3}\right)$, 2.9-3.2 ppm (9H, $\left.-\mathrm{N}-\left(\mathrm{CH}_{3}\right)_{3}\right), 3.3-3.6\left(2 \mathrm{H},-\mathrm{CH}_{2}-\mathrm{N}-\right)$, and 4.0-4.3 (6H, $\left.-\mathrm{CH}_{2}-\mathrm{O}-\right)$.

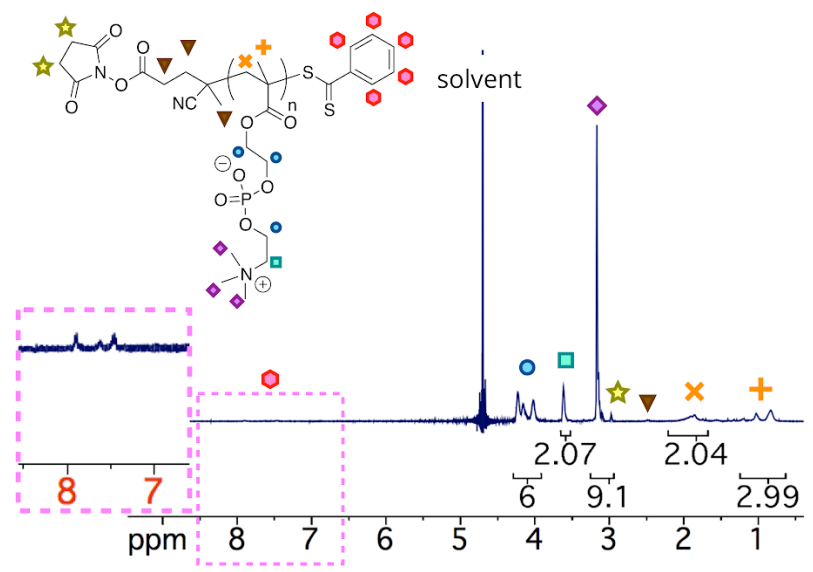

Figure 2. Representative ${ }^{1} \mathrm{H}$ NMR of purified $\mathrm{PMPC}$ in $\mathrm{D}_{2} \mathrm{O}$. 
A representative ${ }^{1} \mathrm{H}$ NMR spectrum of the PMPC- $b$-PVBTMA homopolymer is shown in Figure 3. The calculated chemical composition of VBTMA was determined to be $75 \%$, in good agreement with the targeted chemical composition of VBTMA (68\%). ${ }^{1} \mathrm{H}$ NMR $\left(400 \mathrm{MHz}, \mathrm{D}_{2} \mathrm{O}\right)$ $\delta$ : 0.7-2.1 ppm (alkyl backbone), 2.6-3.0 ppm $\left(9 \mathrm{H},-\mathrm{N}-\left(\mathrm{CH}_{3}\right)_{3}\right), 3.0-3.3 \mathrm{ppm}\left(9 \mathrm{H},-\mathrm{N}-\left(\mathrm{CH}_{3}\right)_{3}\right)$, 3.5-3.7 ppm (2H, $\left.-\mathrm{CH}_{2}-\mathrm{N}-\right)$, 3.8-4.5 ppm (6 H, $\left.-\mathrm{CH}_{2}-\mathrm{O}-; 2 \mathrm{H},-\mathrm{CH}_{2}-\mathrm{N}-\right)$, and 6.1-7.4 ppm (4H, ArH).

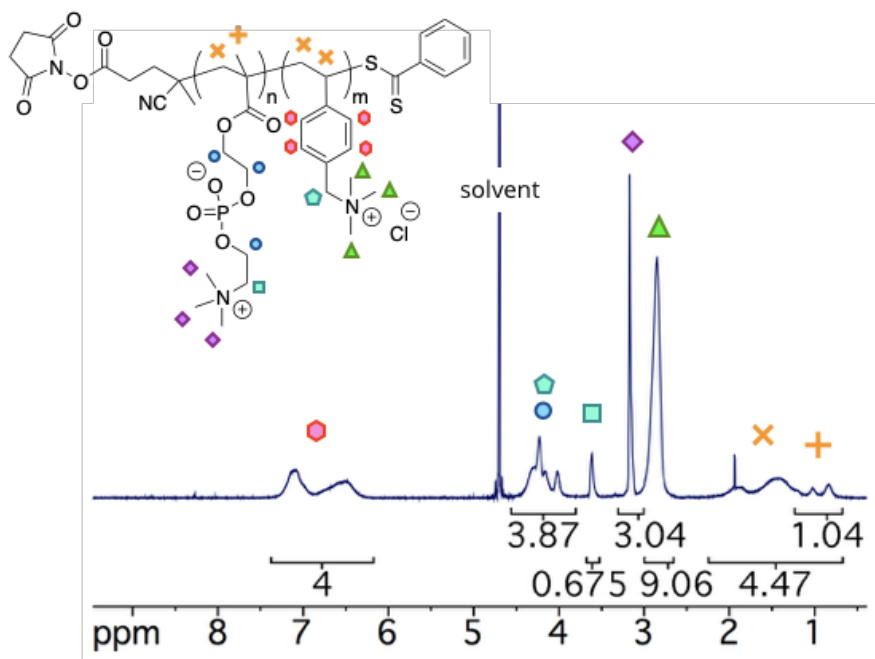

Figure 3. Representative ${ }^{1} \mathrm{H}$ NMR of purified PMPC-PVBTMA in $\mathrm{D}_{2} \mathrm{O}$.

\subsection{Thermogravimetric Analysis (TGA)}

TGA was conducted using a TA Instruments Discovery TGA equipped with an infrared furnace, auto-sampler, and a gas delivery module. Nitrogen was used as the purge gas set at a flow rate of $10 \mathrm{~mL} / \mathrm{min}$; a heating rate of $10{ }^{\circ} \mathrm{C} / \mathrm{min}$ was set for all samples. TA TRIOS software (Version 2.2) was used to analyze the thermal transitions. 


\subsection{Micelle Preparation}

PCMs were prepared via rapid mixing of water, $\mathrm{NaCl}$ or $\mathrm{MgCl}_{2}$, and polyelectrolyte solutions. Water and salt were first mixed with the neutral-cationic diblock polymer, mixed thoroughly with a vortexer, and followed by the polyanion and additional rapid mixing. The polyelectrolytes were added from a $5 \mathrm{mg} / \mathrm{mL}$ stock for a final concentration of $3 \mathrm{mM}$ charge concentration for both the cation and anion. For PEG-PLK and PEG-PVBTMA systems, reversing the order had no effect on the final assembly structure. For PMPC-PVBTMA, we unexpectedly observed a dependency of adding the diblock polycation to the polyanion (or vice versa) on the micelle size and response to salt, as shown in Figure S-38. The origin of this phenomenon is the subject of ongoing investigation.

\subsection{Dynamic Light Scattering (DLS)}

DLS measurements were made using a Brookhaven Instruments BI-200SM Research Goniometer System with an incident laser $(\lambda=637 \mathrm{~nm})$ at room temperature. A dust-free decalin bath was used to match the refractive index of glass. The angular dependence of $D$ was acquired by plotting $q^{2}$ versus decay rate $\Gamma$. A linearity over a range of scattering angles is a good indication of isotropic scatters. The average hydrodynamic radius of scatterers under Brownian diffusion was calculated by the Stokes-Einstein relationship. The correlation function at each angle was fitted to a first-order single exponential using a MATLAB code. The size distribution was obtained using the REPES algorithm. Kinetics experiments were done on the same instrument by recording data every $5 \mathrm{~min}$ for up to $16 \mathrm{~h}$ in a sealed sample container to minimize evaporation.

For the low salt $(0-200 \mathrm{mM} \mathrm{NaCl})$ screening studies, experiments were carried out on a $163.5^{\circ}$ single-angle Wyatt Möbiu $\zeta$ instrument (Santa Barbara, CA) with an incident laser $(\lambda=532$ 
$\mathrm{nm}$ ) at $20^{\circ} \mathrm{C}$. Prepared micelle solutions were directly transferred to quartz micro-cuvettes. The size and colloidal polydispersity index were analyzed using DYNAMICS software.

\subsection{Small-Angle X-ray Scattering (SAXS)}

SAXS measurements were made at beamline 12-ID-B of the Advanced Photon Source (APS) at Argonne National Laboratory (Lemont, IL, USA) and at beamline 4-2 of the Stanford Synchrotron Radiation Lightsource (SSRL) at SLAC National Accelerator Laboratory. Glycerol was added to all samples for a final concentration of $1 \%$ by volume to prevent sample degradation.

For APS experiments, micelle samples were irradiated in a thin-wall glass capillary flow cell with a photon energy of $14 \mathrm{keV}$. Data were reduced in MATLAB at the beamline. Background subtraction and fitting were performed using the multi-level modeling macros distributed with the Irena software package [58] for Igor Pro as described in Ref [56].

For SSRL experiments, the sample-to-detector distance was set to $3.5 \mathrm{~m}$ with measurements collected at a photon energy of $9 \mathrm{keV}$. Aliquots of $30 \mu \mathrm{L}$ of the polyelectrolyte micellar suspension were loaded onto the automated fluid sample loader at the beamline. At least 20 consecutive $1 \mathrm{~s}$ exposures were collected first from the buffer background (water, or water/salt mixtures), followed by samples. During data collection, solutions were oscillated in a stationary quartz capillary cell to maximize the exposed volume and reduce the radiation dose per unit volume. The SAXS data were radially integrated, analyzed for radiation damage, and buffer subtracted using the automated data reduction pipeline available at the beam line. Only data that did not show any evidence of radiation damage were included in the final average for each sample.

\subsection{Micelle Salt Dependence}


Micelles were prepared in numerous salt solutions and compared via SAXS and DLS. High salt concentrations disrupt complex formation as large amounts of counterions compete with ion pairing. This is noticeable as SAXS and DLS intensity decrease and nanoparticles lose shape and dissolve into solution. The salt resistance of a polyelectrolyte complex can be used as a measure of ion pair stability in the assembly material.

\subsection{Micelle Stability Tests}

Micelle solutions were prepared in water with no salt at $4.5 \mathrm{mg} / \mathrm{mL}$ of total polymer. These solutions were then diluted 5x in 100\% Fetal Bovine Serum (FBS), and DLS measurements were taken at $\theta=90^{\circ}$ scattering angle at $25^{\circ} \mathrm{C}$ for 2 min collection time every $5 \mathrm{~min}$. The autocorrelation function and light scattering intensity were recorded for an $8 \mathrm{hr}$ period to observe changes in the size of detectable particles in the presence of FBS media. 


\section{Results}

\subsection{PMPC-PVBTMA Synthesis and Characterization}

We have previously demonstrated the consequences of modifying the chemical nature of the core in PCMs for the complexation of oligonucleotides with PLK or PVBTMA units [55]. Other studies that employ block polyelectrolytes with PEG as the neutral block have also highlighted analogous design considerations [13,59]. However, far fewer PCM studies have focused on the fundamental PCM properties of zwitterionic block polyelectrolytes that form coreshell structured micelles. To this end, we sought to prepare direct polycation analogs to PEG-PLK and PEG-PVBTMA and evaluate aspects of PCM formulation with PAA as a model polyanion. For nomenclature throughout this work, subscripts next to the neutral and charged polymer block represent the molar mass and number-average degree of polymerization, respectively.

As shown in Scheme 1, the RAFT synthesis of PMPC-PVBTMA diblock polyelectrolytes were achieved using CPSE as a suitable RAFT CTA for MPC monomer in deoxygenated methanol at $50{ }^{\circ} \mathrm{C}$, followed by chain extension of the PMPC macromolecular CTA with VBTMA monomer in deoxygenated acetate buffer/ethanol at $50{ }^{\circ} \mathrm{C}$. Each reaction was run overnight $(>18 \mathrm{~h})$. The experimentally measured degree of polymerization (DP) of the charged block was similar to the targeted values, affording $\mathrm{PMPC}_{5 \mathrm{~K}}-\mathrm{PVBTMA}_{50}$ and $\mathrm{PMPC}_{10 \mathrm{~K}}-\mathrm{PVBTMA}_{97}{ }^{1} \mathrm{H}$ NMR studies indicated that the RAFT synthesis in both steps was successful. Additionally, aqueous SEC-MALS showed distinct monomodal peaks of the PMPC precursor and PMPC-PVBTMA diblocks in the optimized mobile phase solutions. Representative SEC refractive index traces are shown in Figure 4. We observe a very small fraction of unreacted PMPC by SEC-MALS, which likely represents dead chains that lost end-group fidelity. 


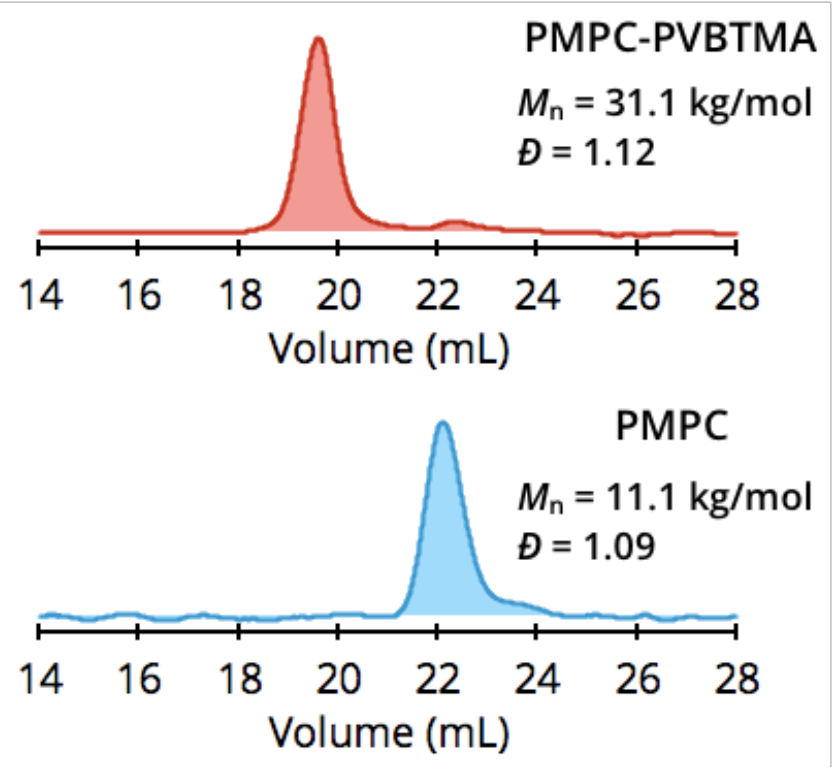

Figure 4. Representative SEC refractive index traces of $\mathrm{PMPC}_{10 \mathrm{~K}}$ (blue curve, bottom) and $\mathrm{PMPC}_{10 \mathrm{~K}}-b$-PVBTMA 100 (red curve, top).

Table 1 summarizes the characterization of the polymer number-average molecular weight $\left(M_{\mathrm{n}}\right)$, dispersity $(\bigoplus)$, and degradation temperature $\left(T_{\mathrm{d}}\right)$. Data on the PEG-PLK samples are reported as received. For all diblock polyelectrolyte systems, the $M_{\mathrm{n}}$ distribution remained relatively narrow $(Đ<1.2)$. By thermogravimetric analysis, two distinct thermal degradations corresponded to each neutral and charged block (Supplementary Figure S-8). Thus, the RAFT chain extension protocols for PEG-PVBTMA and PMPC-PVBTMA were controlled and successful in generating neutralcharged diblock polyelectrolytes. The PAA polyanion was also synthesized with aqueous RAFT chemistry targeting a DP of 50 and fully characterized $\left(M_{\mathrm{n}}=4.9\right.$ and $5.0 \mathrm{~kg} / \mathrm{mol}$ by SEC-MALS and NMR end-group analysis, respectively; $D=1.11$ ). Details of the RAFT synthesis and characterization of PAA are described in the Supplementary Information. In short, these six uniform diblock polycations were used for complexation with PAA to prepare PCMs for solution studies. 
Table 1. Summary of Polymer Characterization.

\begin{tabular}{|c|c|c|c|}
\hline System ${ }^{a}$ & $M_{\mathrm{n}, \operatorname{SEC}}{ }^{\mathrm{b}}(\mathrm{kg} / \mathrm{mol})$ & $\boldsymbol{D}^{\mathrm{c}}$ & $T_{\mathrm{d}}{ }^{\mathrm{d}}\left({ }^{\circ} \mathrm{C}\right)$ \\
\hline $\mathrm{PEG}_{5 \mathrm{~K}}-\mathrm{PLK}_{47}$ & 13.0 & 1.06 & 314,420 \\
\hline $\mathrm{PEG}_{10 \mathrm{~K}}-\mathrm{PLK}_{93}$ & 25.6 & 1.06 & 320,404 \\
\hline $\mathrm{PEG}_{5 \mathrm{~K}}-\mathrm{PVBTMA}{ }_{53}$ & 18.3 & 1.12 & 199,378 \\
\hline $\mathrm{PEG}_{10 \mathrm{~K}}-\mathrm{PVBTMA_{100 }}$ & 29.1 & 1.17 & 212,366 \\
\hline $\mathrm{PMPC}_{5 \mathrm{~K}}-\mathrm{PVBTMA}_{50}$ & 27.8 & 1.12 & 211,461 \\
\hline $\mathrm{PMPC}_{10 \mathrm{~K}-\mathrm{PVBTMA} 97}$ & 40.0 & 1.10 & 218,460 \\
\hline $\mathrm{PAA}_{50}$ & 4.9 & 1.11 & N/A \\
\hline
\end{tabular}

a Subscripts denote the molar mass of the PEG/PMPC block and the degree of polymerization of the PLK/PVBTMA block from ${ }^{1} \mathrm{H}$ NMR spectroscopy, respectively. Characterization of $\mathrm{PEG}_{5 \mathrm{~K}}-\mathrm{PLK}_{47}$ and $\mathrm{PEG}_{10 \mathrm{~K}}-\mathrm{PLK}_{93}$ are reported from the manufacturer. ${ }^{b}$ Experimentally-measured number-average molecular weight, determined by SEC-MALS. ${ }^{\mathrm{c}}$ Dispersity $(\nexists)=M_{\mathrm{w}} / M_{\mathrm{n} .}{ }^{\mathrm{d}}$ Degradation temperature $\left(T_{\mathrm{d}}\right)$ by TGA.

3.2 Polyelectrolyte Complex Micelle (PCM) and Zwitterionic PCM (zPCM) Assembly, Salt Resistance, and Stability

In order to elucidate the effect these physical and chemical differences have on micelle structure, we used the six polymers from Table 1 to separately complex with PAA(50) as a common polyanion, to form micelles in water. PCMs and $\mathrm{zPCMs}$ were assembled via direct dissolution and rapid mixing into the desired salt solution. Three polymer chemistries are being compared (PEG-PLK, PEG-PVBTMA, and PMPC-PVBTMA) at two lengths each (i.e., a 10 $\mathrm{kg} / \mathrm{mol}$ neutral block with $\sim 100$ unit cation versus a $5 \mathrm{~kg} / \mathrm{mol}$ neutral block and $\sim 50$ unit cation). For nomenclature, these systems will be referred to as neutral(10k)-charged(100) and neutral(5k)charged(50). First, we screened the assembly size and size distribution of PEG-PVBTMA and PMPC-PVBTMA systems as a function of salt with a single-angle dynamic light scattering (DLS) 
instrument. In a low salt regime from $0-75 \mathrm{mM} \mathrm{NaCl}$, no difference in salt resistance was detected (Supplementary Figure S-9). At $100 \mathrm{mM} \mathrm{NaCl}$, we observed an increase in the polydispersity of the zPCMs for both neutral(10k)-charged(100) and neutral(5k)-charged(50) lengths. In comparison, the PCMs for both systems with an outer PEG corona exhibited no change in aggregation through $200 \mathrm{mM} \mathrm{NaCl}$.

To better understand this salt-driven transition in PCM and zPCM assembly, small angle X-ray scattering (SAXS) was used to more precisely quantify the micelle shape and the size of the core and corona, providing complete structural morphology for each system. Figure 5 shows SAXS results for each neutral(10k)-charged(100) system in $100 \mathrm{mM} \mathrm{NaCl}$. For all six systems, we observe micelle formation at $100 \mathrm{mM} \mathrm{NaCl}$. PEG-PVBTMA and PMPC-PVBTMA systems form spherical micelles, apparent from the flat slope at low q, while PEG-PLK forms worm-like micelles $\left(\mathrm{q}^{-2}\right.$ dependence) when complexed with PAA. We also notice a consistent core size difference where PMPC-PVBTMA is the largest and PEG-PLK is the smallest, determined by SAXS fitting results displayed in Table 2 in the next section below. This was consistent with the DLS screening results. Furthermore, longer cationic blocks form larger micelle cores throughout this study, consistent with previous reports [52,56] of micelle size being solely dependent on the charged block length of the block copolymer and independent of homopolymer length. 

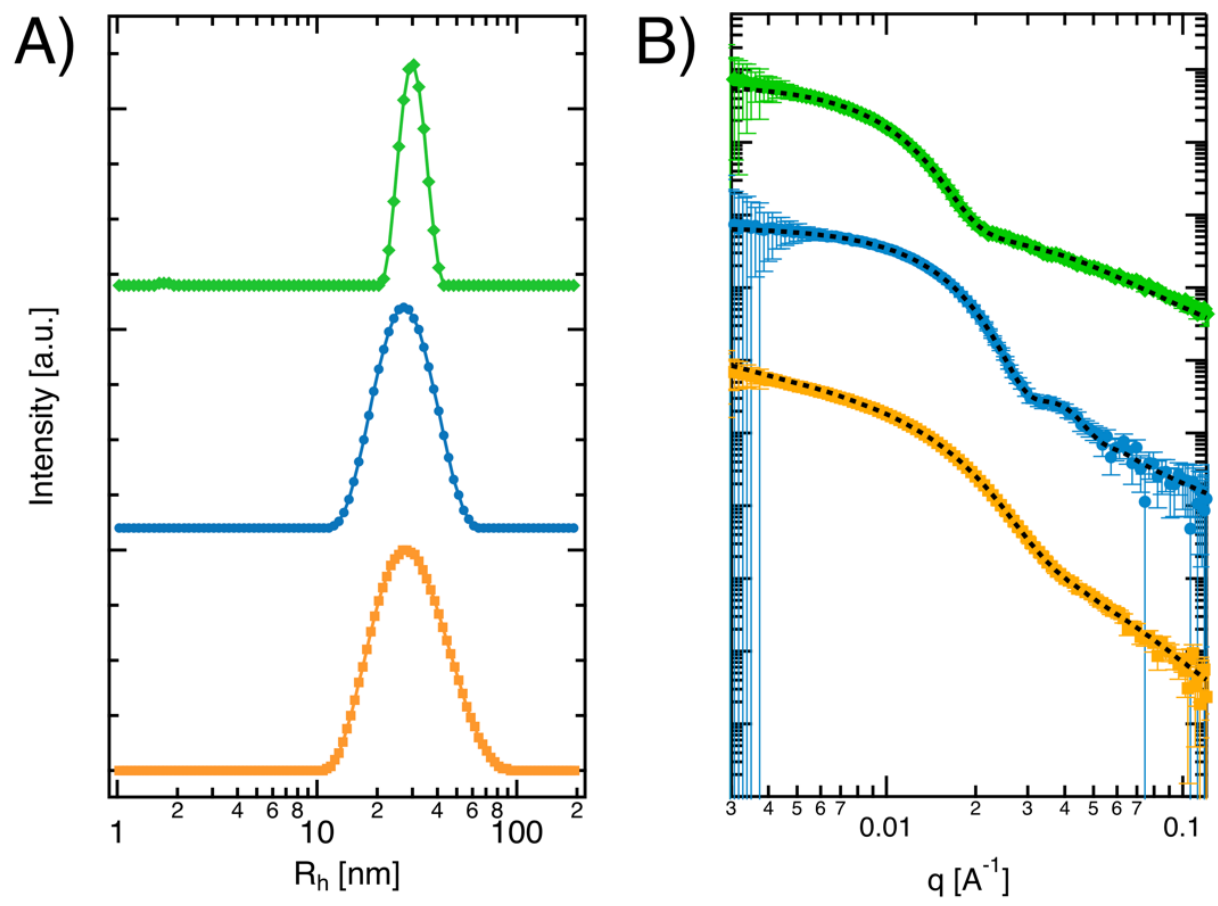

- PMPC-PVBTMA

- PEG-PLK

Figure 5. Comparison of the A) apparent size hydrodynamic radius distribution of neutral(10k)charged(100) micelles at $100 \mathrm{mM} \mathrm{NaCl}$, analyzed by REPES analysis at the $90^{\circ}$ angle, and the $\mathrm{B}$ ) corresponding small angle X-ray scattering data (colored markers) and fits (black dashed lines). Intensity scaled for clarity; from bottom to up in both plots, the systems correspond to PEG-PLK (orange squares), PEG-PVBTMA (blue circles), and PMPC-PVBTMA (green diamonds).

Next, we aimed to evaluate the salt resistance and stability of the prepared PCMs and zPCMs with multi-angle DLS and SAXS modeling. We focused on the $0 \mathrm{mM}$ and $100 \mathrm{mM} \mathrm{NaCl}$ conditions as a point of comparison. For both salt concentrations across all six systems, the hydrodynamic radius $\left(R_{\mathrm{h}}\right)$ and colloidal polydispersity $\left(\mathrm{PDI}=\mu_{2} / \Gamma^{2}\right)$ were determined by fitting the autocorrelation function to a cumulant expansion [60] and via regularized positive exponential sum (REPES) analysis [61] (Supplementary Table S-1). Excellent agreement in micelle size and 
low polydispersity between both methods were reported, again with a consistent hydrodynamic size difference where PMPC-PVBTMA was the largest and PEG-PLK was the smallest.

Recent reports have emphasized the importance of the charged group and neutral block chemistry for influencing complex micelle stability. In general, stability can be quantified by salt resistance - the critical salt concentration at which a complex assembly comes apart and dissolves into solution due to the abundance of ions in solution. In other words, electrostatic interactions are screened, thereby reducing the effective charge density holding the nanocarrier assembly together. In the individual polyelectrolyte solutions of PEG-PLK, PEG-PVBTMA, and PMPC-PVBTMA, there was no observed difference in the disappearance of the signature correlation peak that is prominent in the SAXS profile by adding up to $500 \mathrm{mM} \mathrm{NaCl}$ (Supplementary Figure S-40). This suggests that the conformation of the neutral-charged polycation chains respond to salt in a similar manner. In order to probe the stability of these systems complexed with PAA(50) and their behavior in higher concentrations of monovalent and divalent salts, we formed PCMs and zPCMs in 100,250 , and $500 \mathrm{mM} \mathrm{NaCl}$, as well as 50 and $100 \mathrm{mM} \mathrm{MgCl}_{2}$.

Figure 6 shows the SAXS results for the neutral(10k)-charged(100) cases, and fitting results are available in Table 2 . The data for neutral(5k)-charged(50) systems show similar trends and can be found in Supplementary Figures S40-S45. For PEG-PLK PCMs, minimal structural differences or changes in scattering intensity were seen across all conditions, signifying strong complexation. PEG-PVBTMA formed PCMs at 100 and $250 \mathrm{mM} \mathrm{NaCl}$ and $50 \mathrm{mM} \mathrm{MgCl}_{2}$ but not at higher salts. PMPC-PVBTMA exhibited the weakest complexation, with well-formed zPCMs only at $100 \mathrm{mM} \mathrm{NaCl}$. Additionally, both PVBTMA systems revealed a loss in intensity and increase in size dispersity with increased salt, which was not present in the PEG-PLK system. PLK has been shown to form stronger ion pairs with DNA in previous literature [55], and here we 
observe a consistent trend with PAA. We find a drastic discrepancy in salt stability due to the neutral versus zwitterionic block. PMPC-PVBTMA is unable to form micelles at $50 \mathrm{mM} \mathrm{MgCl} 2$ and forms extremely polydisperse poorly formed micelles at $250 \mathrm{mM} \mathrm{NaCl}$, conditions that are favorable for micelle formation when using the PEG variant. Additional SAXS data and models are available in Supplementary Figures S40-S45.
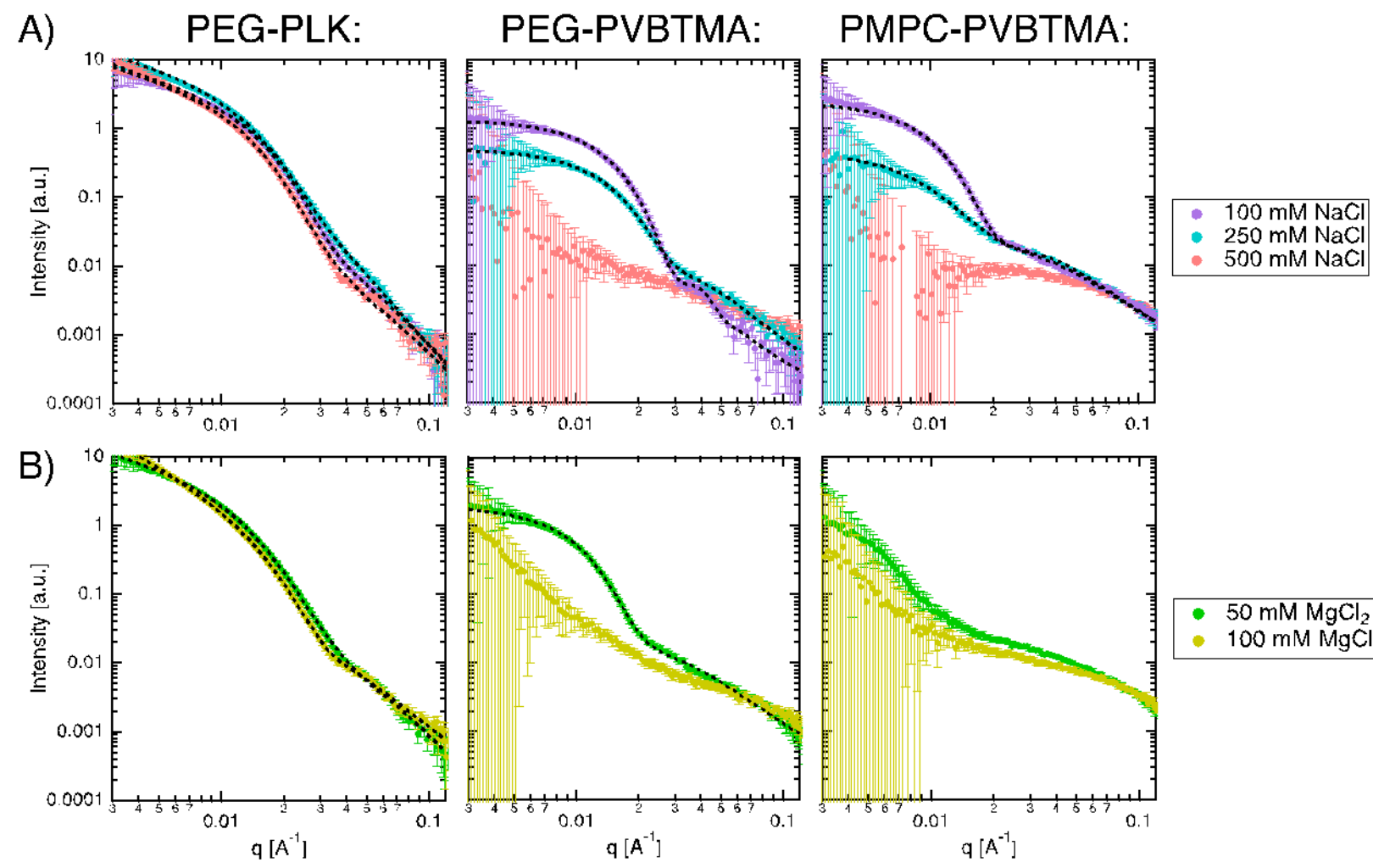

$50 \mathrm{mM} \mathrm{MgCl} 2$ $100 \mathrm{mM} \mathrm{MgCl}_{2}$

Figure 6. Small angle X-ray scattering data (color) and fits (black) for neutral(10k)-charged(100) micelles in a range of A) $\mathrm{NaCl}$ and $\mathrm{B}) \mathrm{MgCl}_{2}$ concentrations. PEG-PLK micelles are most resistant to salt, followed by PEG-PVBTMA, then PMPC-PVBTMA. Data are not scaled for this figure to show decreasing intensity with added salt.

Table 2. SAXS fit parameters summary with added $\mathrm{NaCl}$ salt. 


\begin{tabular}{|c|c|c|c|c|}
\hline Sample & {$[\mathrm{NaCl}](\mathbf{m M})$} & $\mathbf{R}^{\mathrm{a}}(\mathbf{n m})$ & PDI $^{b}$ & Aspect Ratio \\
\hline \multirow{3}{*}{$\mathrm{PEG}_{5 \mathrm{~K}}-\mathrm{PLK}_{47} / \mathrm{PAA}_{50}$} & 100 & 7.2 & 0.14 & - \\
\hline & 250 & 6.3 & 0.22 & - \\
\hline & 500 & 8.7 & 0.13 & - \\
\hline \multirow{3}{*}{$\mathrm{PEG}_{10 \mathrm{~K}}-\mathrm{PLK}_{93} / \mathrm{PAA}_{50}$} & 100 & 10.8 & 0.08 & - \\
\hline & 250 & 10.7 & 0.15 & - \\
\hline & 500 & 11.8 & 0.10 & - \\
\hline \multirow{3}{*}{$\mathrm{PEG}_{5 \mathrm{~K}}-\mathrm{PVBTMA}_{53} / \mathrm{PAA}_{50}$} & 100 & 12.3 & 0.03 & 1.4 \\
\hline & 250 & 9.8 & 0.23 & 1.03 \\
\hline & 500 & - & - & - \\
\hline \multirow{3}{*}{$\mathrm{PEG}_{10 \mathrm{~K}}-\mathrm{PVBTMA}_{100} / \mathrm{PAA}_{50}$} & 100 & 13.7 & 0.01 & 1.65 \\
\hline & 250 & 12.9 & 0.07 & 1.24 \\
\hline & 500 & - & - & - \\
\hline \multirow{3}{*}{$\mathrm{PMPC}_{5 \mathrm{~K}}-\mathrm{PVBTMA} 50$ / PAA50 } & 100 & 17.7 & 0.03 & 1.69 \\
\hline & 250 & 16.2 & 0.09 & 1.17 \\
\hline & 500 & - & - & - \\
\hline \multirow{3}{*}{$\mathrm{PMPC}_{10 \mathrm{~K}}-\mathrm{PVBTMA}_{97} / \mathrm{PAA}_{50}$} & 100 & 19.4 & 0.05 & 1.36 \\
\hline & 250 & 10.0 & 0.44 & 1.11 \\
\hline & 500 & - & - & - \\
\hline
\end{tabular}

${ }^{a}$ Mean radius $(\mathrm{nm})^{\mathrm{b}}$ Polydispersity index $\left(\sigma^{2} / \mathrm{R}^{2}\right)$. Models use Schulz-Zimm Spheroid and Unified Level fits, except for PEG5K-PLK 47 / PAA50 (flexible cylinder fitting) and PEG $10 \mathrm{~K}-\mathrm{PLK}_{93}$ / PAA50 (cylinder fitting).

\subsection{PCMs and zPCMs in Biologically Relevant Media}

Since micelle stability is crucial for systemic circulation and controlled release, we investigated the structural integrity of prepared PCMs and zPCMs in biologically relevant media, FBS. The temporal evolution of the micelles in full serum was monitored with static scattering intensity (I). As previously shown by Lin and coworkers [62], this technique provides a straightforward way to detect micelle-protein interactions shown in Equation 1: 
$K$ is the prefactor constant from the optics setup, $c$ is the sample mass concentration, and $M_{\mathrm{w}}$ is the weight-average molecular weight of the suspended sample. The amount of light scattered is directly proportional to the molar mass and concentration of nanoparticles in solution. Because the pristine micelles that were prepared from all polyelectrolyte pairings are larger than the serum proteins in the FBS control sample (Figure S-46), the total $I$ will increase if the $M_{\mathrm{w}}$ increases as a result of strong associative interactions between proteins and micelles.

For representative neutral(10k)-charged(100) PEG-PLK, PEG-PVBTMA, and PMPCPVBTMA micelles in FBS, Figure 7 provides a summary of the average change in $I$ over the course of $8 \mathrm{~h}$, along with the apparent size distribution at 1 and $10 \mathrm{~h}$. Experiments were conducted multiple times to ensure repeatability (see Figure S-46 for repeats). The FBS negative control showed no evidence of aggregation or sedimentation over $8 \mathrm{~h}$. It should be noted that FBS media exhibits a broad bimodal distribution, so the PCMs cannot be distinguished from serum proteins. In the stability tests, the total $I$ for both the PEG-PLK and PEG-PVBTMA systems remained relatively constant, while the PMPC-PVBTMA system increased by approximately $10 \%$ in $I$ on average with greater variation over time. REPES analysis of the PMPC-PVBTMA system shows the growth of $\sim 200 \mathrm{~nm}$ aggregates over 1 to $10 \mathrm{~h}$. This slight stability issue may be mitigated by increasing the degree of polymerization of PMPC, as the total length of the zwitterionic corona in micelles have been previously shown to affect nanoparticle stabilization and delivery efficacy [33]. Nevertheless, because proteins adsorption onto nanoparticle carriers in biological settings is a known design consideration for micelle assemblies [63,64], the prepared PCMs and zPCM show good overall resistance to destabilization by serum. 

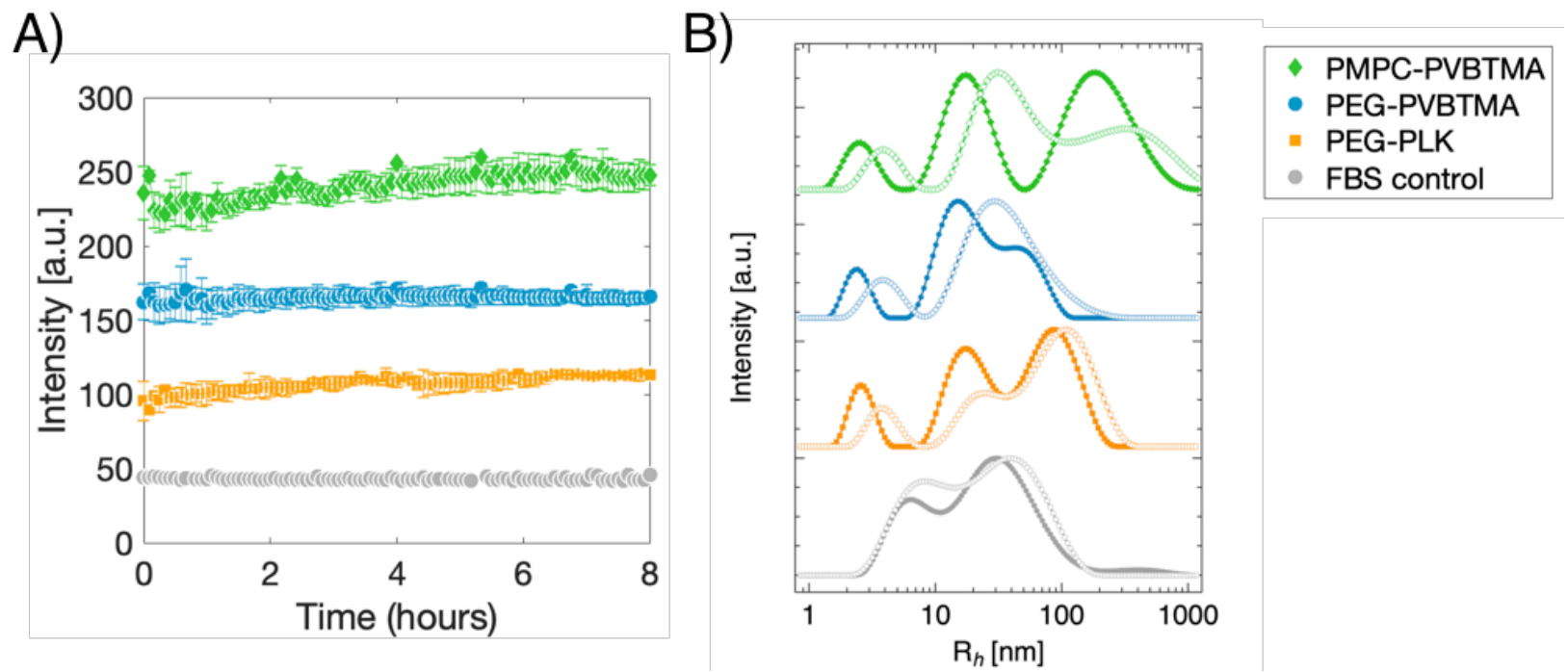

Figure 7. A) Total light scattering intensity at $90^{\circ}$ angle over $8 \mathrm{~h}$ of $100 \%$ FBS (gray circles) and 1:5 dilution of PEG-PLK (orange squares), PEG-PVBTMA (blue circles), and PMPC-PVBTMA (green diamonds) micelles with 100\% FBS. Data points and error bars denote the average and standard deviation of 2 replicates; intensity is scaled for clarity. B) The apparent size hydrodynamic radius distribution of respective micelles at $1 \mathrm{~h}$ (solid markers) and $10 \mathrm{~h}$ (open markers), analyzed by REPES analysis at the $90^{\circ}$ angle. Intensity is scaled for clarity.

\section{Discussion}

In alignment with greater efforts reported recently on elucidating the interplay between electrostatics and other non-covalent associations on polyelectrolyte complexation $[65,66]$, this work provides a central platform for designing charged micelle-based nanocarriers with fundamental structure-property relationships. Neutral blocks in PCMs and zPCMs force nanophase separation and provide a protective corona around a charged polymer core, which often contains a sequestered cargo. Understanding the physical effects attributed to zwitterionic and neutral polymer coronas, and chemically distinct charged polymer cores, expands the design space 
and versatility of PCMs and zPCMs. Altogether, we have prepared a series of polyelectrolytebased nanocarriers from PEG-PLK, PEG-PVBTMA, and PMPC-PVBTMA with PAA, characterized the self-assembled micelle structure in water as a function of salt, and evaluated long-term stability in biologically relevant media. Aqueous RAFT polymerization was used to synthesize a well-defined zwitterionic diblock polyelectrolyte system PMPC-PVBTMA at various block lengths. Upon complexation with PAA, the zPCMs exhibited $\sim 40 \mathrm{~nm}$ spherical micelles. These uniform assemblies were slightly larger than the PCMs formed from PEG-PLK and PEGPVBTMA $(\sim 20-30 \mathrm{~nm})$.

Characterizing micelle structure and disassembly in the presence of monovalent and divalent salts provide a measure of complex stability. Our results show that complexes formed by PEG-PLK are much more stable against salt, followed by PEG-PVBTMA which is slightly more resistant to salt than PMPC-PVBTMA, the least stable system. This result is consistent for both neutral(5k)-charged(50) and neutral(10k)-charged(100) block lengths. Overall, this study examines neutral and zwitterionic chemistries used as polymer coronas in PCMs to protect sequestered cargo and to control particle size, stability, and environmental responsiveness.

Supplementary Materials: The following are available online: polymer synthesis data, dynamic light scattering data and fitting results, and small-angle X-ray scattering data and fitting results.

Author Contributions: Conceptualization: A.E.M. and J.M.T.; Polymer synthesis and characterization: J.M.T. and J.D.M.; X-ray scattering and analysis: A.E.M. and J.M.T. Light scattering and analysis: A.E.M., J.M.T., J.D.M., and T.C. Research direction and funding: M.V.T. All authors participated in interpreting the data and preparing the manuscript. 
Funding: This work was performed under the following financial assistance award 70NANB19H005 from U.S. Department of Commerce, National Institute of Standards and Technology as part of the Center for Hierarchical Materials Design (CHiMaD). J.M.T. acknowledges support from the NIST-CHiMaD Postdoctoral Fellowship. This work used resources of the Advanced Photon Source, a U.S. Department of Energy (DOE) Office of Science User Facility operated for the DOE Office of Science by Argonne National Laboratory under Contract No. DE-AC02-06CH11357. Use of the Stanford Synchrotron Radiation Lightsource, SLAC National Accelerator Laboratory, is supported by the U.S. Department of Energy, Office of Science, Office of Basic Energy Sciences under Contract No. DE-AC02-76SF00515. The SSRL Structural Molecular Biology Program is supported by the DOE Office of Biological and Environmental Research, and by the National Institutes of Health, National Institute of General Medical Sciences (P41GM103393). The contents of this publication are solely the responsibility of the authors and do not necessarily represent the official views of NIGMS or NIH.

Acknowledgements: Parts of this work were carried out at the Soft Matter Characterization Facility at University of Chicago, Advanced Photon Source at Argonne National Laboratory, and the Stanford Synchrotron Radiation Lightsource at SLAC National Accelerator Laboratory. The authors gratefully thank Xiaobing Zuo and Thomas Weiss for their assistance with the SAXS data acquisition and Prof. Amanda B. Marciel for helpful discussions on polyampholytes.

Conflicts of Interest: The authors declare no conflict of interest. 


\section{REFERENCES}

1. Muthukumar, M. 50th Anniversary Perspective: A Perspective on Polyelectrolyte Solutions. Macromolecules 2017, 50, 9528-9560.

2. Sing, C. E. Micro- to macro-phase separation transition in sequence-defined coacervates. $J$. Chem. Phys. 2020, 152, 024902.

3. Acar, H.; Ting, J. M.; Srivastava, S.; LaBelle, J. L.; Tirrell, M. V. Molecular engineering solutions for therapeutic peptide delivery. Chem. Soc. Rev. 2017, 46, 6553-6569.

4. Voets, I. K.; de Keizer, A.; Stuart, M. A. C. Complex coacervate core micelles. Adv. Colloid Interface Sci. 2009, 147-148, 300-318.

5. Marciel, A. B.; Chung, E. J.; Brettmann, B. K.; Leon, L. Bulk and nanoscale polypeptide based polyelectrolyte complexes. Adv. Colloid Interface Sci. 2017, 239, 187-198.

6. Brangwynne, C. P.; Tompa, P.; Pappu, R. V. Polymer physics of intracellular phase transitions. Nat. Phys. 2015, 11, 899-904.

7. Cooper, C. L.; Dubin, P. L.; Kayitmazer, A. B.; Turksen, S. Polyelectrolyte-protein complexes. Curr. Opin. Colloid Interface Sci. 2005, 10, 52-78.

8. Horn, J.; Kapelner, R.; Obermeyer, A. Macro- and Microphase Separated ProteinPolyelectrolyte Complexes: Design Parameters and Current Progress. Polymers 2019, 11, 578.

9. Harada, A.; Kataoka, K. Effect of Charged Segment Length on Physicochemical Properties of Core-Shell Type Polyion Complex Micelles from Block Ionomers. Macromolecules 2003, 36, 4995-5001.

10. van der Kooij, H. M.; Spruijt, E.; Voets, I. K.; Fokkink, R.; Cohen Stuart, M. A.; van der Gucht, J. On the Stability and Morphology of Complex Coacervate Core Micelles: From Spherical to Wormlike Micelles. Langmuir 2012, 28, 14180-14191. 
11. Lemmers, M.; Voets, I. K.; Cohen Stuart, M. A.; Gucht, der, J. V. Transient network topology of interconnected polyelectrolyte complex micelles. Soft Matter 2011, 7, 1378-1389.

12. Srivastava, S.; Andreev, M.; Levi, A. E.; Goldfeld, D. J.; Mao, J.; Heller, W. T.; Prabhu, V. M.; de Pablo, J. J.; Tirrell, M. V. Gel phase formation in dilute triblock copolyelectrolyte complexes. Nat. Comm. 2017, 8, 14131.

13. Heo, T.-Y.; Kim, I.; Chen, L.; Lee, E.; Lee, S.; Choi, S.-H. Effect of Ionic Group on the Complex Coacervate Core Micelle Structure. Polymers 2019, 11, 455.

14. Rahalkar, A.; Wei, G.; Nieuwendaal, R.; Prabhu, V. M.; Srivastava, S.; Levi, A. E.; de Pablo, J. J.; Tirrell, M. V. Effect of temperature on the structure and dynamics of triblock polyelectrolyte gels. J. Chem. Phys. 2018, 149, 163310.

15. Knop, K.; Hoogenboom, R.; Fischer, D.; Schubert, U. S. Poly(ethylene glycol) in Drug Delivery: Pros and Cons as Well as Potential Alternatives. Angew. Chem. Int. Ed. 2010, 49, 62886308.

16. Ishihara, T.; Takeda, M.; Sakamoto, H.; Kimoto, A.; Kobayashi, C.; Takasaki, N.; Yuki, K.; Tanaka, K.-I.; Takenaga, M.; Igarashi, R.; Maeda, T.; Yamakawa, N.; Okamoto, Y.; Otsuka, M.; Ishida, T.; Kiwada, H.; Mizushima, Y.; Mizushima, T. Accelerated Blood Clearance Phenomenon Upon Repeated Injection of PEG-modified PLA-nanoparticles. Pharm. Res. 2009, 26, 2270-2279. 17. Ishihara, T.; Maeda, T.; Sakamoto, H.; Takasaki, N.; Shigyo, M.; Ishida, T.; Kiwada, H.; Mizushima, Y.; Mizushima, T. Evasion of the Accelerated Blood Clearance Phenomenon by Coating of Nanoparticles with Various Hydrophilic Polymers. Biomacromolecules 2010, 11, $2700-2706$.

18. Pelegri-O’Day, E. M.; Lin, E.-W.; Maynard, H. D. Therapeutic Protein-Polymer Conjugates: Advancing Beyond PEGylation. J. Am. Chem. Soc. 2014, 136, 14323-14332. 
19. Van Bruggen, C.; Hexum, J. K.; Tan, Z.; Dalal, R. J.; Reineke, T. M. Nonviral Gene Delivery with Cationic Glycopolymers. Acc. Chem. Res. 2019, 52, 1347-1358.

20. Elsabahy, M.; Wooley, K. L. Design of polymeric nanoparticles for biomedical delivery applications. Chem. Soc. Rev. 2012, 41, 2521-3012.

21. Burke, P. A.; Pun, S. H.; Reineke, T. M. Advancing Polymeric Delivery Systems Amidst a Nucleic Acid Therapy Renaissance. ACS Macro Lett. 2013, 2, 928-934.

22. Zou, H.; Wang, Z.; Feng, M. Nanocarriers with tunable surface properties to unblock bottlenecks in systemic drug and gene delivery. J. Control. Release 2015, 214, 121-133.

23. Hoang Thi, T. T.; Pilkington, E. H.; Nguyen, D. H.; Lee, J. S.; Park, K. D.; Truong, N. P. The Importance of Poly(ethylene glycol) Alternatives for Overcoming PEG Immunogenicity in Drug Delivery and Bioconjugation. Polymers 2020, 12, 298.

24. Blackman, L. D.; Gunatillake, P. A.; Cass, P.; Locock, K. E. S. An introduction to zwitterionic polymer behavior and applications in solution and at surfaces. Chem. Soc. Rev. 2019, 6, 757-770. 25. Schlenoff, J. B. Zwitteration: Coating Surfaces with Zwitterionic Functionality to Reduce Nonspecific Adsorption. Langmuir 2014, 30, 9625-9636.

26. Ishihara, K. Revolutionary advances in 2-methacryloyloxyethyl phosphorylcholine polymers as biomaterials. J. Biomed. Mater. Res. 2019, 107, 933-943.

27. Kitano, H.; Sudo, K.; Ichikawa, K.; Ide, M.; Ishihara, K. Raman Spectroscopic Study on the Structure of Water in Aqueous Polyelectrolyte Solutions. J. Phys. Chem. B 2000, 104, 1142511429.

28. Ladd, J.; Zhang, Z.; Chen, S.; Hower, J. C.; Jiang, S. Zwitterionic Polymers Exhibiting High Resistance to Nonspecific Protein Adsorption from Human Serum and Plasma. Biomacromolecules 2008, 9, 1357-1361. 
29. Goda, T.; Ishihara, K.; Miyahara, Y. Critical update on 2-methacryloyloxyethyl phosphorylcholine (MPC) polymer science. J. Appl. Polym. Sci. 2015, 132, 41766.

30. Sae-ung, P.; Kolewe, K. W.; Bai, Y.; Rice, E. W.; Schiffman, J. D.; Emrick, T.; Hoven, V. P. Antifouling Stripes Prepared from Clickable Zwitterionic Copolymers. Langmuir 2017, 33, 70287035 .

31. He, Q.; Qiao, Y.; Mandia, D. J.; Gan, S.; Zhang, H.; Zhou, H.; Elam, J. W.; Darling, S. B.; Tirrell, M. V.; Chen, W. Enrichment and Distribution of Pb2+ Ions in Zwitterionic Poly (cysteine methacrylate) Brushes at the Solid-Liquid Interface. Langmuir 2019, 35, 17082-17089.

32. Lewis, A.; Tang, Y.; Brocchini, S.; Choi, J.-W.; Godwin, A. Poly(2-methacryloyloxyethyl phosphorylcholine) for Protein Conjugation. Bioconjugate Chem. 2008, 19, 2144-2155.

33. Jackson, M. A.; Werfel, T. A.; Curvino, E. J.; Yu, F.; Kavanaugh, T. E.; Sarett, S. M.; Dockery, M. D.; Kilchrist, K. V.; Jackson, A. N.; Giorgio, T. D.; Duvall, C. L. Zwitterionic Nanocarrier Surface Chemistry Improves siRNA Tumor Delivery and Silencing Activity Relative to Polyethylene Glycol. ACS Nano 2017, 11, 5680-5696.

34. Cao, Z.; Zhang, G. Dynamics of polyzwitterions in salt-free and salt solutions. Phys. Chem. Chem. Phys. 2015, 17, 27045-27051.

35. Georgiev, G. S.; Kamenska, E. B.; Vassileva, E. D.; Kamenova, I. P.; Georgieva, V. T.; Iliev, S. B.; Ivanov, I. A. Self-Assembly, Antipolyelectrolyte Effect, and Nonbiofouling Properties of Polyzwitterions. Biomacromolecules 2006, 7, 1329-1334.

36. Shao, Q.; Jiang, S. Molecular Understanding and Design of Zwitterionic Materials. Adv. Mater. 2014, 27, 15-26. 
37. Kobayashi, M.; Terayama, Y.; Kikuchi, M.; Takahara, A. Chain dimensions and surface characterization of superhydrophilic polymer brushes with zwitterion side groups. Soft Matter 2013, 9, 5138-5148.

38. Morozova, S.; Hu, G.; Emrick, T.; Muthukumar, M. Influence of Dipole Orientation on Solution Properties of Polyzwitterions. ACS Macro Lett. 2016, 5, 118-122.

39. Wang, J.; Yuan, S.; Zhang, Y.; Wu, W.; Hu, Y.; Jiang, X. The effects of poly(zwitterions)s versus poly(ethylene glycol) surface coatings on the biodistribution of protein nanoparticles. Biomater. Sci. 2016, 4, 1351-1360.

40. Keefe, A. J.; Jiang, S. Poly(zwitterionic)protein conjugates offer increased stability without sacrificing binding affinity or bioactivity. Nat. Chem. 2011, 4, 59-63.

41. Miyamoto, D.; Watanabe, J.; Ishihara, K. Effect of water-soluble phospholipid polymers conjugated with papain on the enzymatic stability. Biomaterials 2004, 25, 71-76.

42. Lowe, A. B.; McCormick, C. L. Synthesis and Solution Properties of Zwitterionic Polymers. Chem. Rev. 2002, 102, 4177-4190.

43. Lobb, E. J.; Ma, I.; Billingham, N. C.; Armes, S. P.; Lewis, A. L. Facile Synthesis of WellDefined, Biocompatible Phosphorylcholine-Based Methacrylate Copolymers via Atom Transfer Radical Polymerization at $20^{\circ}$ C. J. Am. Chem. Soc. 2001, 123, 7913-7914.

44. Hsiue, G.-H.; Lo, C.-L.; Cheng, C.-H.; Lin, C.-P.; Huang, C.-K.; Chen, H.-H. Preparation and characterization of poly(2-methacryloyloxyethyl phosphorylcholine)-block-poly(D,L-lactide) polymer nanoparticles. J. Polym. Sci. A Polym. Chem. 2007, 45, 688-698.

45. Matsuda, Y.; Kobayashi, M.; Annaka, M.; Ishihara, K.; Takahara, A. Dimension of poly (2methacryloyloxyethyl phosphorylcholine) in aqueous solutions with various ionic strength. Chem. Lett. 2006, 35, 1310-1311. 
46. Topham, P. D.; Sandon, N.; Read, E. S.; Madsen, J.; Ryan, A. J.; Armes, S. P. Facile Synthesis of Well-Defined Hydrophilic Methacrylic Macromonomers Using ATRP and Click Chemistry. Macromolecules 2008, 41, 9542-9547.

47. Bhuchar, N.; Deng, Z.; Ishihara, K.; Narain, R. Detailed study of the reversible additionfragmentation chain transfer polymerization and co-polymerization of 2-methacryloyloxyethyl phosphorylcholine. Polym. Chem. 2011, 2, 632-639.

48. Nakaura, H.; Kawamura, A.; Miyata, T. Reductively Responsive Gel Capsules Prepared Using a Water- Soluble Zwitterionic Block Copolymer Emulsifier. Langmuir 2018, 35, 1413-1420.

49. Takahashi, R.; Narayanan, T.; Yusa, S.-I.; Sato, T. Kinetics of Morphological Transition between Cylindrical and Spherical Micelles in a Mixture of Anionic-Neutral and Cationic-Neutral Block Copolymers Studied by Time-Resolved SAXS and USAXS. Macromolecules 2018, 51, $3654-3662$.

50. Ting, J. M.; Wu, H.; Herzog-Arbeitman, A.; Srivastava, S.; Tirrell, M. V. Synthesis and Assembly of Designer Styrenic Diblock Polyelectrolytes. ACS Macro Lett. 2018, 7, 726-733.

51. Wu, H.; Ting, J. M.; S.; Yu, B.; Jackson, N. E.; Rumyantsev, A. M.; Meng, S.; de Pablo, J. J.; Tirrell, M. V. "In situ Time-Resolved Small-Angle X-ray Scattering Reveals the Formation Kinetics of Polyelectrolyte Complex Micelles" ChemRxiv 2019. DOI: 10.26434/chemrxiv.9876395

52. Wu, H.; Ting, J. M.; Weiss, T. M.; Tirrell, M. V. Interparticle Interactions in Dilute Solutions of Polyelectrolyte Complex Micelles. ACS Macro Lett. 2019, 8, 819-825.

53. Wu, H.; Ting, J. M.; Tirrell, M. V. Mechanism of Dissociation Kinetics in Polyelectrolyte Complex Micelles. Macromolecules 2020, 53, 102-111. 
54. Wu, H.; Ting, J. M.; Werba, O.; Meng, S.; Tirrell, M. V. Non-equilibrium phenomena and kinetic pathways in self-assembled polyelectrolyte complexes. J. Chem. Phys. 2018, 149, 163330. 55. Marras, A. E.; Vieregg, J. R.; Ting, J. M.; Rubien, J. D.; Tirrell, M. V. Polyelectrolyte Complexation of Oligonucleotides by Charged Hydrophobic-Neutral Hydrophilic Block Copolymers. Polymers 2019, 11, 83.

56. Marras, A. E.; Vieregg, J. R.; Tirrell, M. V. Assembly and Characterization of Polyelectrolyte Complex Micelles. ChemRxiv 2019. DOI: 10.26434/chemrxiv.11328173.v1

57. Moad, G. RAFT polymerization to form stimuli-responsive polymers. Polym. Chem. 2017, 8, $177-219$.

58. Ilavsky, J.; Jemian, P. R. Irena: tool suite for modeling and analysis of small- angle scattering. J. Appl. Cryst. 2009, 42, 347-353.

59. Krogstad, D. V.; Lynd, N. A.; Miyajima, D.; Gopez, J.; Hawker, C. J.; Kramer, E. J.; Tirrell, M. V. Structural Evolution of Polyelectrolyte Complex Core Micelles and Ordered-Phase Bulk Materials. Macromolecules 2014, 47, 8026-8032.

60. Koppel, D. E. Analysis of Macromolecular Polydispersity in Intensity Correlation Spectroscopy: The Method of Cumulants. J. Chem. Phys. 1972, 57, 4814-4820.

61. J, J. Regularized Positive Exponential Sum (REPES) Program-A Way of Inverting Laplace Transform Data Obtained by Dynamic Light Scattering. Collect. Czech. Chem. Comm. 1995, 60, $1781-1797$.

62. Yin, L.; Dalsin, M. C.; Sizovs, A.; Reineke, T. M.; Hillmyer, M. A. Glucose-Functionalized, Serum-Stable Polymeric Micelles from the Combination of Anionic and RAFT Polymerizations. Macromolecules 2012, 45, 4322-4332. 
63. Galmarini, S.; Hanusch, U.; Giraud, M.; Cayla, N.; Chiappe, D.; Moos, von, N.; Hofmann, H.; Maurizi, L. Beyond Unpredictability: The Importance of Reproducibility in Understanding the Protein Corona of Nanoparticles. Bioconjugate Chem. 2018, 29, 3385-3393.

64. Gupta, M. N.; Roy, I. How Corona Formation Impacts Nanomaterials as Drug Carriers. Mol. Pharmaceutics 2020. Article ASAP. DOI: 10.1021/acs.molpharmaceut.9b01111

65. Huang, J.; Morin, F. J.; Laaser, J. E. Charge-Density-Dominated Phase Behavior and Viscoelasticity of Polyelectrolyte Complex Coacervates. Macromolecules 2019, 52, 4957-4967.

66. Tabandeh, S.; Leon, L. Engineering Peptide-Based Polyelectrolyte Complexes with Increased Hydrophobicity. Molecules 2019, 24, 868. 


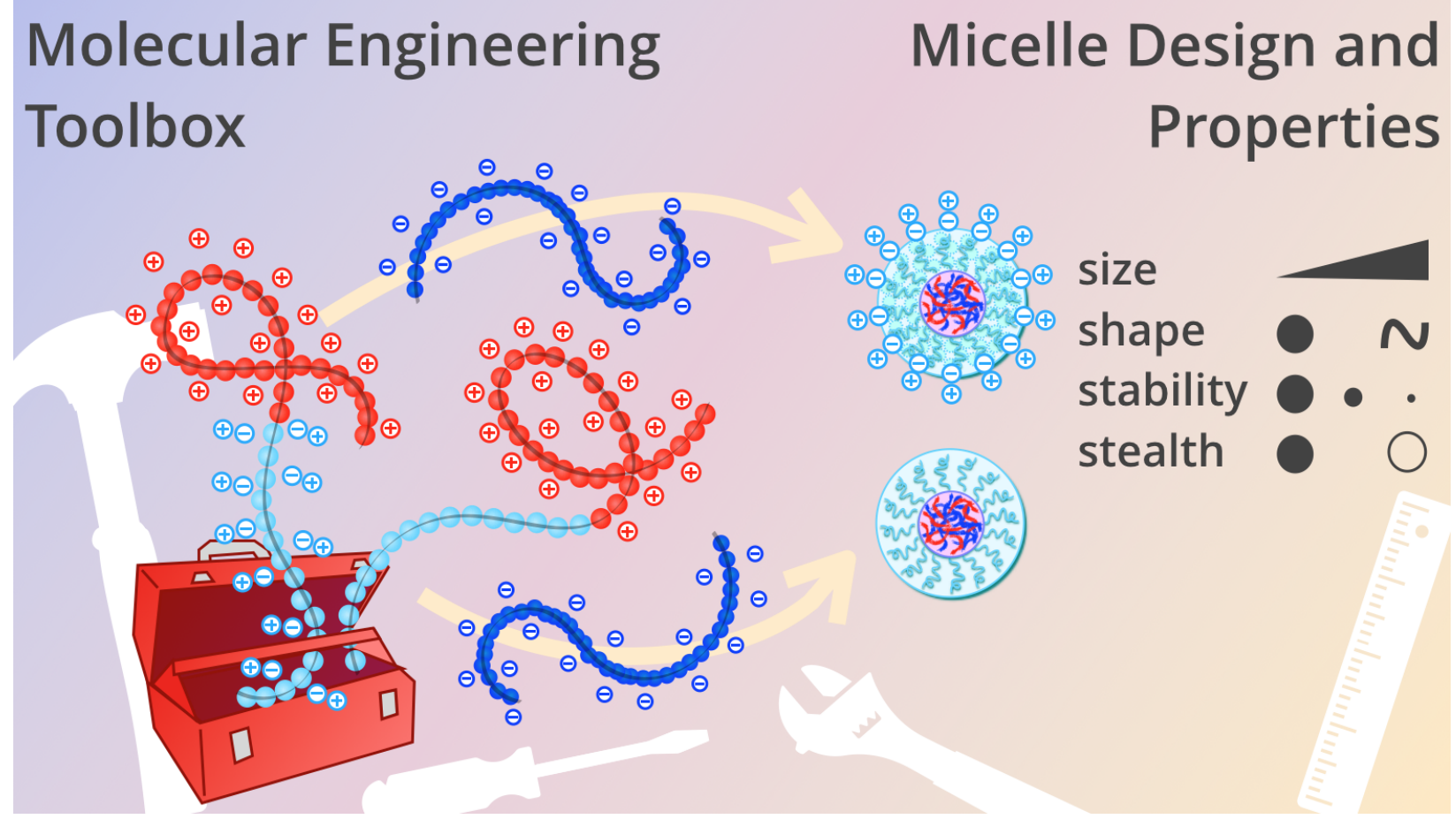


Supporting Information

\section{Comparing Zwitterionic and PEG Exteriors of}

\section{Polyelectrolyte Complex Micelles}

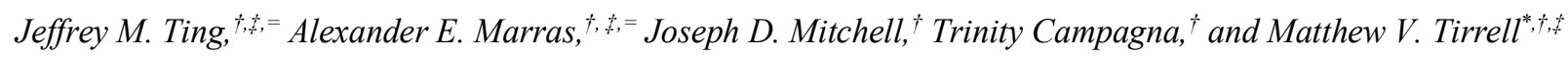

$\dagger$ Pritzker School of Molecular Engineering, University of Chicago, Chicago, Illinois 60637, United States. $\ddagger$ Center for Molecular Engineering, Argonne National Laboratory, Lemont, Illinois 60439, United States.

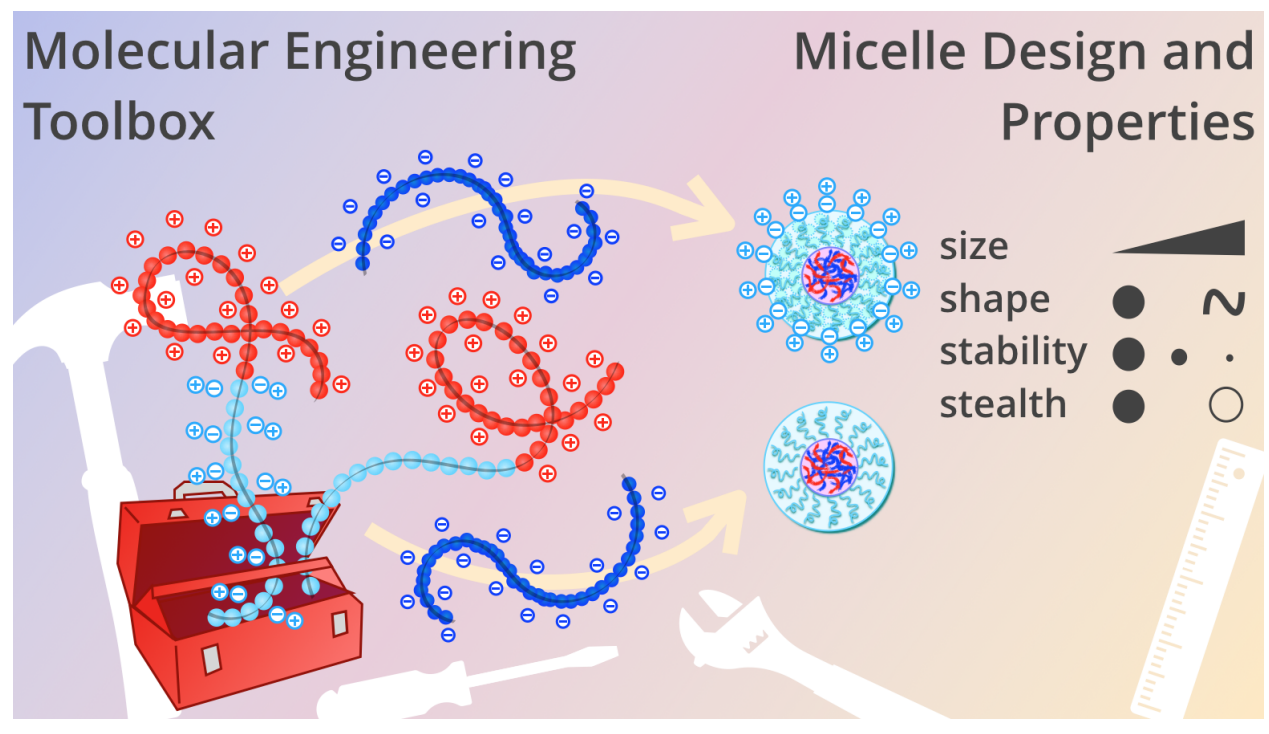

\section{Contents}

S1. Supplemental Polymer Synthesis Data (S-2 to S-6)

S2. Supplemental Dynamic Light Scattering Data (S-7 to S-22)

S3. Supplemental Small-Angle X-Ray Scattering Data (S-23 to S-X)

S4. Supplemental Polyelectrolyte Complex Micelle Stability Data (S-X to S-X) 


\section{S1. Supplemental Polymer Synthesis Data}

PMPC-PVBTMA Synthesis. Figure S-1 shows a representative crude ${ }^{1} \mathrm{H}$ NMR of PMPC, resulting in $81 \%$ total monomer conversion after $18 \mathrm{~h}$. Figure S-2 shows a representative crude ${ }^{1} \mathrm{H}$ NMR of PMPC-PVBTMA, resulting in 93\% total monomer conversion after $18 \mathrm{~h}$. Figure S-3 shows the purified ${ }^{1} \mathrm{H}$ NMR of $\mathrm{PMPC}_{5 \mathrm{~K}}-\mathrm{PVBTMA}_{50}$.

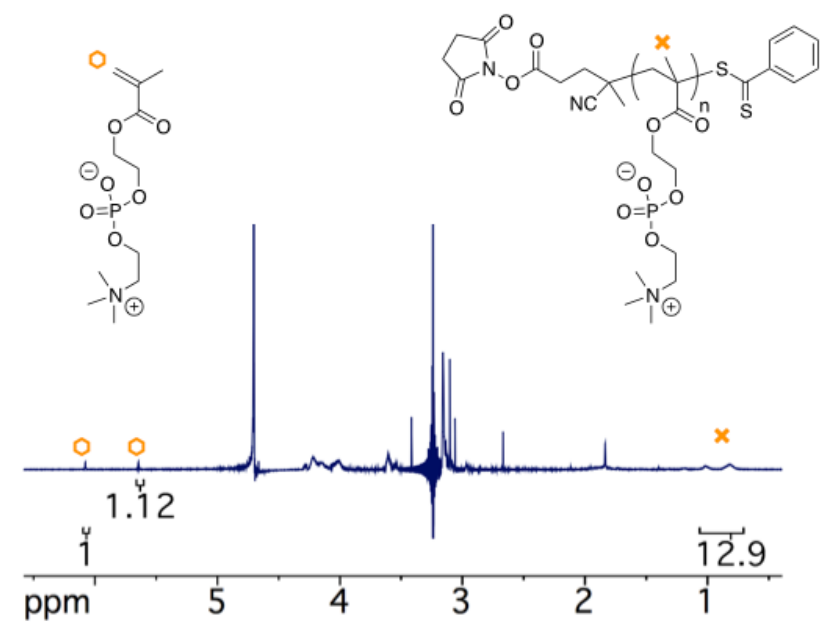

Figure S-1. Crude ${ }^{1} \mathrm{H}$ NMR of PMPC in $\mathrm{D}_{2} \mathrm{O}$.

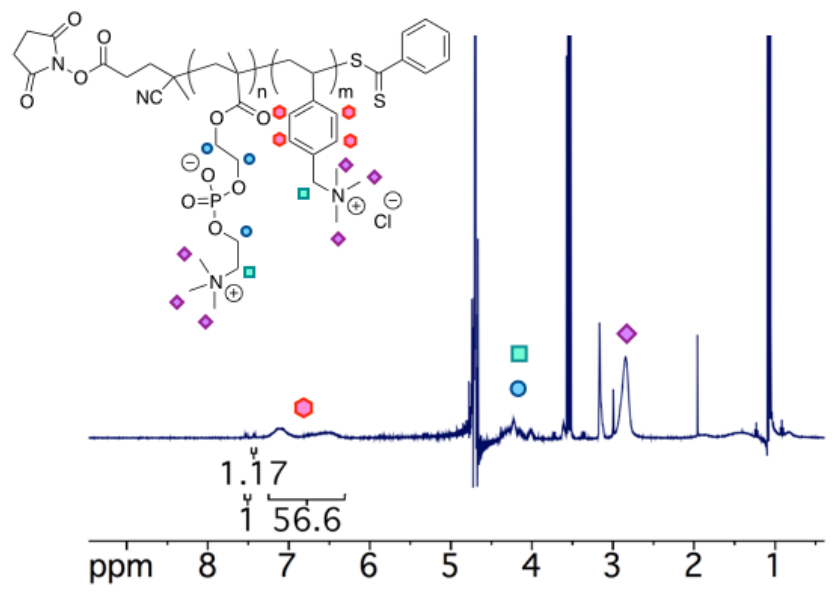

Figure S-2. Crude ${ }^{1} \mathrm{H}$ NMR of PMPC-PVBTMA in $\mathrm{D}_{2} \mathrm{O}$. 


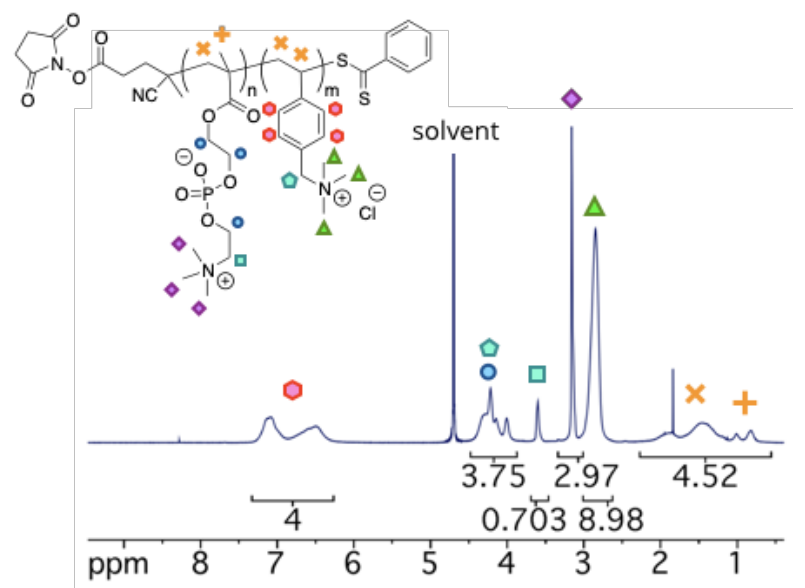

Figure S-3. ${ }^{1} \mathrm{H}$ NMR of $\mathrm{PMPC}_{5 \mathrm{~K}}-\mathrm{PVBTMA}_{50}$ in $\mathrm{D}_{2} \mathrm{O}$.

PAA Synthesis. Figures S-4 and S-5 show the ${ }^{1} \mathrm{H}$ NMR of the BuPA RAFT CTA and the homopolymer PAA prepared with aqueous RAFT polymerization, respectively. End-group analysis $(51 \times 94.04 \mathrm{~g} / \mathrm{mol}+238.39 \mathrm{~g} / \mathrm{mol})$ resulted in a calculated $M_{\mathrm{n}}=5030 \mathrm{~g} / \mathrm{mol}$, in excellent agreement with SEC-MALS characterization. Figure S-6 shows the SEC refractive index trace of PAA, which consists of a monomodal peak exhibiting narrow dispersity in the $M_{\mathrm{n}}$ distribution.

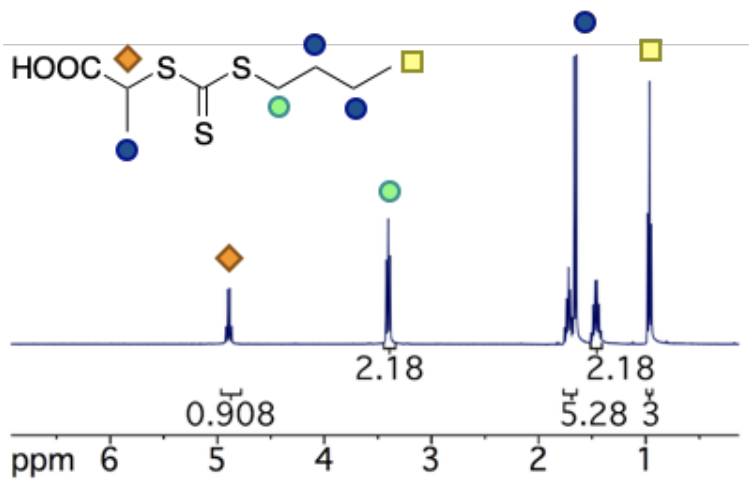

Figure S-4. ${ }^{1} \mathrm{H} \mathrm{NMR}$ of $\mathrm{BuPA}$ in $\mathrm{CDCl}_{3}$. 


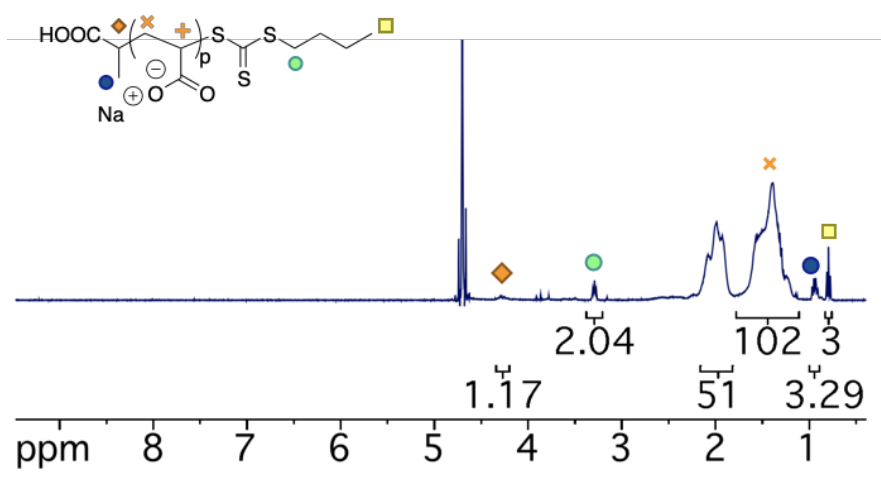

Figure S-5. ${ }^{1} \mathrm{H}$ NMR of PAA in $\mathrm{D}_{2} \mathrm{O}$.

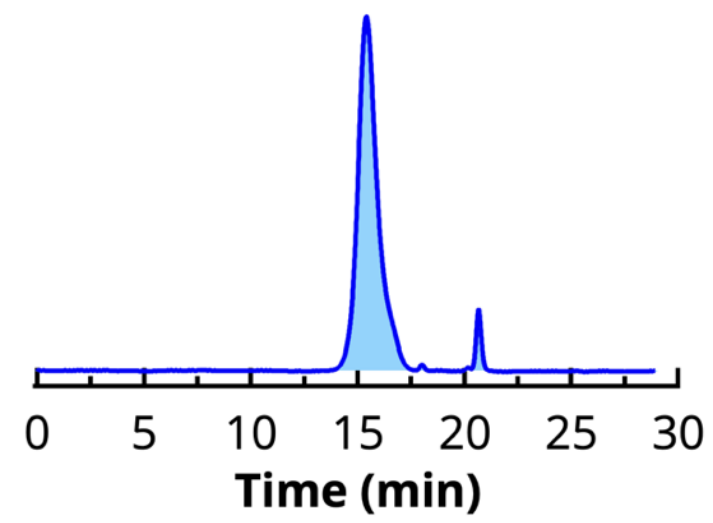

Figure S-6. SEC refractive index trace of PAA $\left(M_{\mathrm{n}}=4900 \mathrm{~g} / \mathrm{mol}, Ð=1.11\right)$.

Refractive Index Measurements. To determine the absolute $M_{\mathrm{n}}$ of the polymers measured by SEC-MALS, we employed a refractometer to measure the $\mathrm{d} n / \mathrm{dc}$ of polymers in their respective mobile phase. For PMPC-PVBTMA samples, Figure S-7 shows the $\mathrm{d} n / \mathrm{dc}$ determination of the individual homopolymers. Using Equation 1 shown in the main manuscript, we calculated the $\mathrm{d} n / \mathrm{dc}$ of the $\mathrm{PMPC}_{5 \mathrm{~K}}-\mathrm{PVBTMA}_{50}$ and $\mathrm{PMPC}_{10 \mathrm{~K}}-\mathrm{PVBTMA}_{100}$ to be 0.1661 and $0.1663 \mathrm{~mL} / \mathrm{g}$, respectively. 

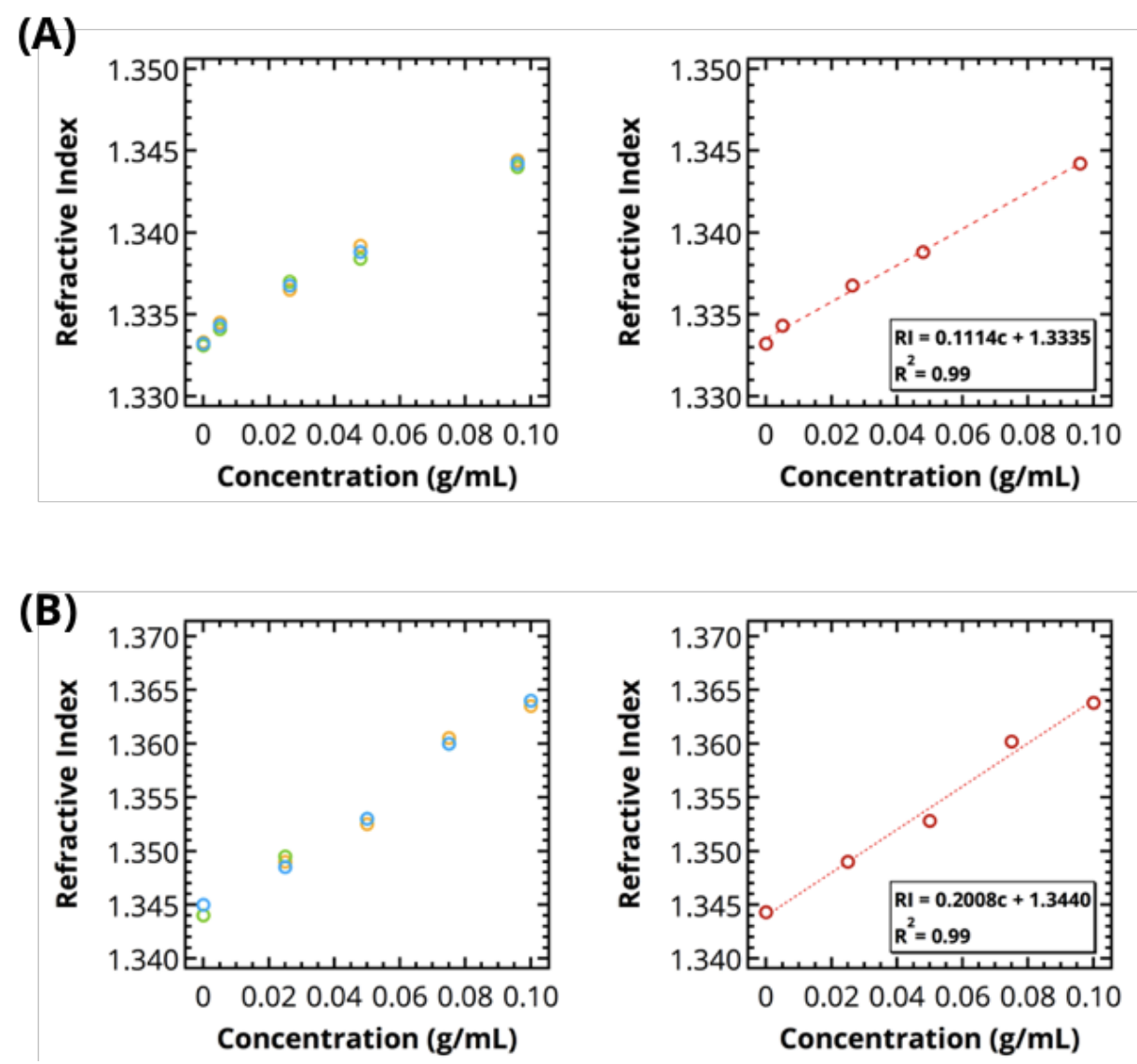

Figure S-7. Measured refractive index values versus polymer concentration of (A) PMPC and (B) PVBTMA. All measurements were taken using polymers completely dissolved in the cationic mobile phase solution at $25{ }^{\circ} \mathrm{C}$. The circles on the left plot show the raw data; the circles on the right plot show the average of triplicate measurements with the dashed line denoting a linear regression to determine $\mathrm{d} n / \mathrm{dc}$.

Thermogravimetric Analysis Curves. Figure S-8 shows the TGA profiles of synthesized polyelectrolytes. All experiments were conducted at $15^{\circ} \mathrm{C} / \mathrm{min}$. 
(A)

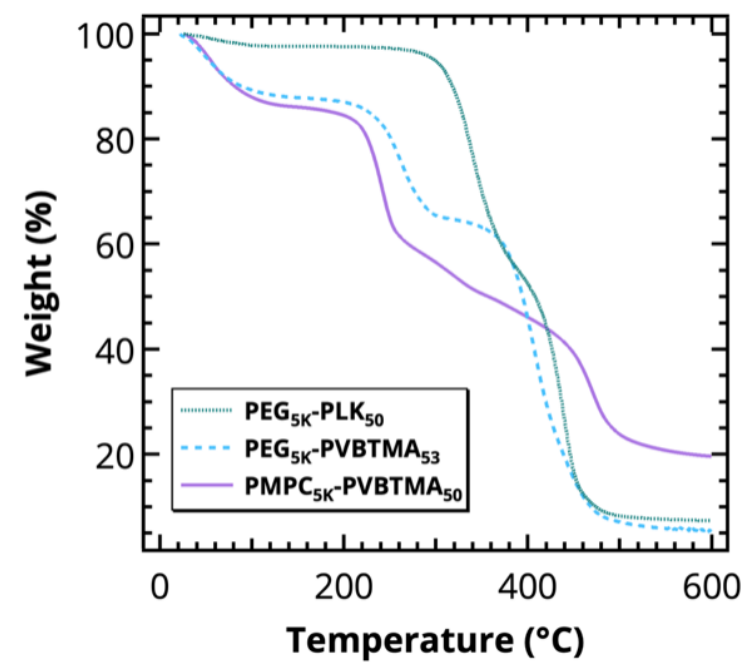

(B)

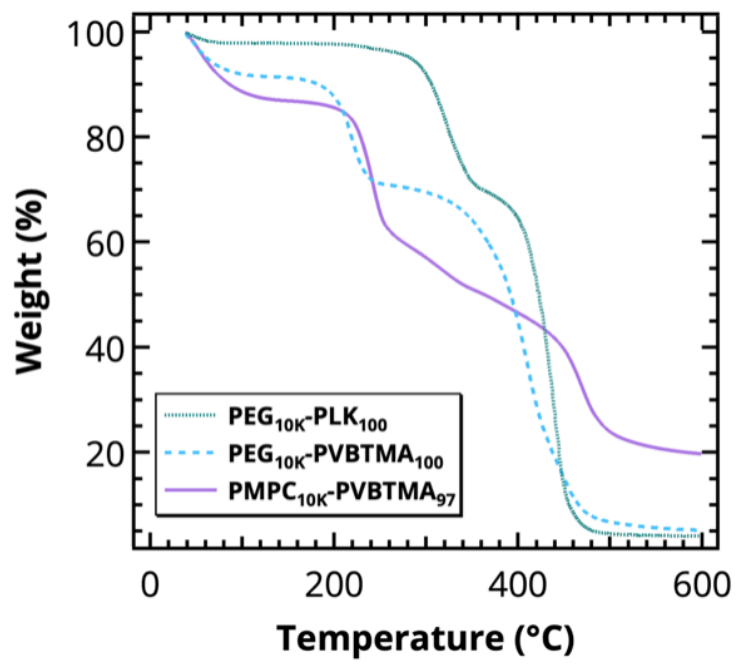

Figure S-8. TGA curves for block polymers (A) $\mathrm{PEG}_{5 \mathrm{~K}}-\mathrm{PLK}_{50}, \mathrm{PEG}_{5 \mathrm{~K}}-\mathrm{PVBTMA}_{53}$ and $\mathrm{PMPC}_{5 \mathrm{~K}}-$ PVBTMA 50 , as well as (B) PEG $10 \mathrm{~K}-\mathrm{PLK}_{100}, \mathrm{PEG}_{10 \mathrm{~K}}-\mathrm{PVBTMA}_{100}$ and PMPC $10 \mathrm{~K}-\mathrm{PVBTMA} 97$. All experiments were conducted at $15^{\circ} \mathrm{C} / \mathrm{min}$. 


\section{S2. Supplemental Dynamic Light Scattering Data}

Figure S-9 shows a gallery of histograms for all prepared PCMs from $0 \mathrm{mM} \mathrm{NaCl}$ to 200 $\mathrm{mM} \mathrm{NaCl}$. The autocorrelation functions for these samples are shown in the Figures S-35 through S-38 below.

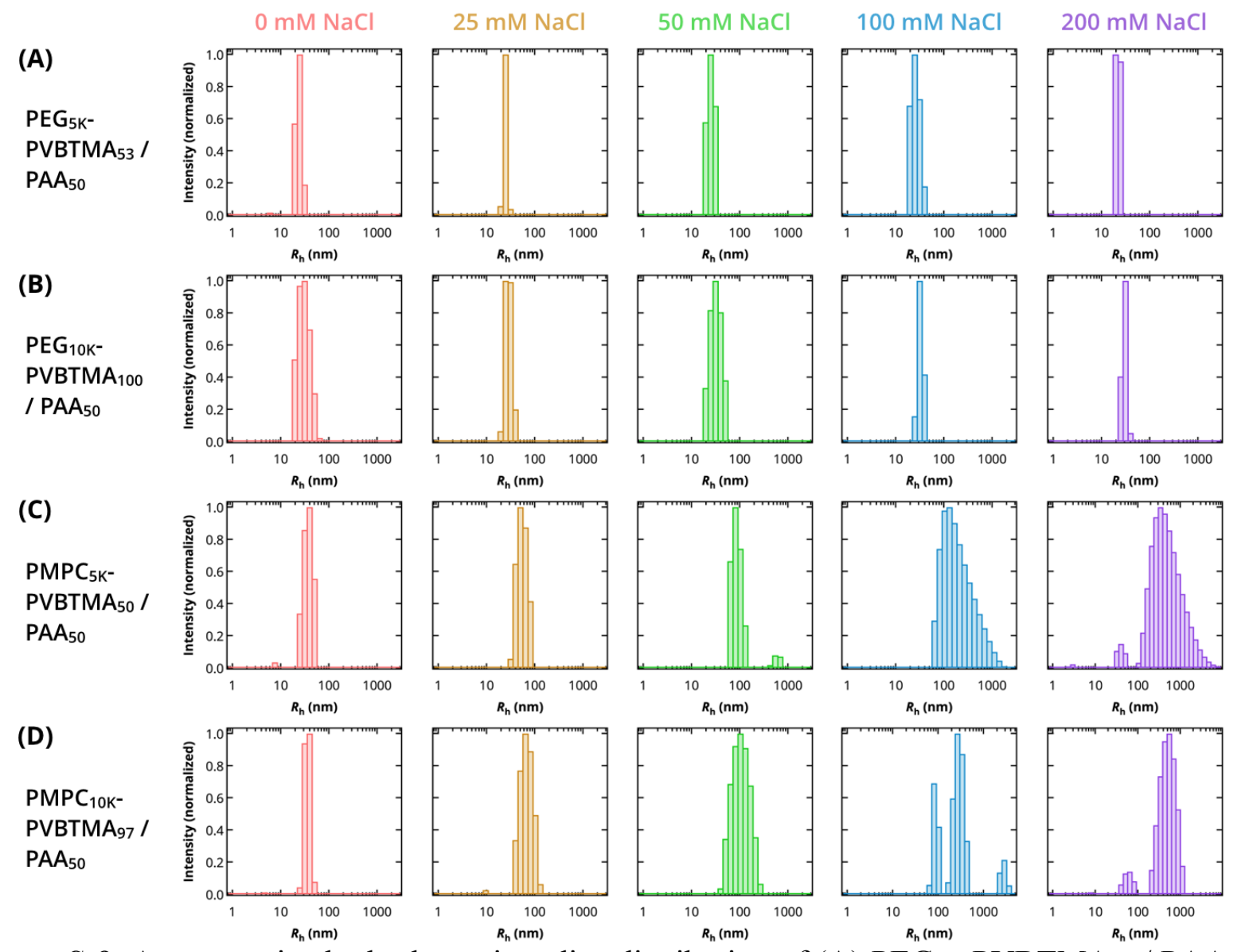

Figure S-9. Apparent size hydrodynamic radius distribution of (A) $\mathrm{PEG}_{5 \mathrm{~K}}-\mathrm{PVBTMA}_{53} / \mathrm{PAA}_{50}$, (B) $\mathrm{PEG}_{10 \mathrm{~K}}-\mathrm{PVBTMA}_{100} / \mathrm{PAA}_{50}$, (C) $\mathrm{PMPC}_{5 \mathrm{~K}}-\mathrm{PVBTMA}_{50} / \mathrm{PAA}_{50}$, and (D) $\mathrm{PMPC}_{10 \mathrm{~K}}$ $\mathrm{PVBTMA}_{97} / \mathrm{PAA}_{50}$ as $\mathrm{NaCl}$ salt is increased from $0 \mathrm{mM}$ to $200 \mathrm{mM}$ (left to right).

Table S-1 summarizes the multi-angle DLS experiments for all prepared PCMs at $0 \mathrm{mM}$ $\mathrm{NaCl}$ and $100 \mathrm{mM} \mathrm{NaCl}$. The autocorrelation functions were analyzed with a cumulant expansion as well as REPES analysis at the $90^{\circ}$ scattering angle. 
Table S-1. DLS summary of all prepared PCMs at $0 \mathrm{mM} \mathrm{NaCl}$.

\begin{tabular}{|c|c|c|c|c|}
\hline Sample & $R_{\mathrm{h}}{ }^{\mathrm{a}}(\mathrm{nm})$ & PDI $^{b}$ & $\begin{array}{c}\text { Mean } R_{\mathrm{h}, \text { REPES }}{ }^{\mathrm{c}} \\
(\mathrm{nm})\end{array}$ & $\begin{array}{c}S D R_{\mathrm{h}, \text { REPES }} \\
(\mathrm{nm})\end{array}$ \\
\hline $\mathrm{PEG}_{5 \mathrm{~K}}-\mathrm{PLK}_{47} / \mathrm{PAA}_{50}$ & $16.4 \pm 0.1$ & $0.18 \pm 0.03$ & 18.6 & 2.8 \\
\hline $\mathrm{PEG}_{10 \mathrm{~K}}-\mathrm{PLK}_{93} / \mathrm{PAA}_{50}$ & $21.1 \pm 0.2$ & $0.11 \pm 0.03$ & 22.8 & 1.3 \\
\hline $\mathrm{PEG}_{5 \mathrm{~K}}-\mathrm{PVBTMA}_{53} / \mathrm{PAA}_{50}$ & $20.9 \pm 0.8$ & $0.28 \pm 0.07$ & 24.1 & 4.1 \\
\hline $\mathrm{PEG}_{10 \mathrm{~K}}-\mathrm{PVBTMA} \mathrm{A}_{100} / \mathrm{PAA}_{50}$ & $27.2 \pm 0.7$ & $0.26 \pm 0.04$ & 29.4 & 6.1 \\
\hline $\mathrm{PMPC}_{5 \mathrm{~K}}-\mathrm{PVBTMA}{ }_{50} / \mathrm{PAA}_{50}$ & $40.0 \pm 1.4$ & $0.22 \pm 0.01$ & 50.7 & 11.4 \\
\hline $\mathrm{PMPC}_{10 \mathrm{~K}}-\mathrm{PVBTMA} 97$ / PAA 50 & $42.0 \pm 1.6$ & $0.23 \pm 0.03$ & 52.9 & 11.7 \\
\hline
\end{tabular}

${ }^{a}$ Mean hydrodynamic radius $\left(R_{\mathrm{h}}\right) \pm 95 \%$ CI from a cumulant expansion across $60-120^{\circ} .{ }^{\mathrm{b}}$ Mean colloidal polydispersity (PDI $\left.=\mu_{2} / \Gamma^{2}\right) \pm 95 \% \mathrm{CI}$ from a cumulant expansion across $60-120^{\circ}$. ${ }^{\mathrm{c}}$ Average $R_{\mathrm{h}}$ determined by REPES analysis at the $90^{\circ}$ scattering angle. ${ }^{\mathrm{d}}$ Standard deviation of $R_{\mathrm{h}}$ distribution from REPES analysis at the $90^{\circ}$ scattering angle.

The detailed multi-angle DLS analysis of the PCMs is provided below. For each polymer system, Figures S-9 through S-32 show the measured angular dependence of the autocorrelation functions between $60^{\circ}$ and $120^{\circ}$ fitted by a cumulant expansion, as well as the linear regression of $\Gamma$ vs $q^{2}$ between $60^{\circ}$ and $120^{\circ}$.
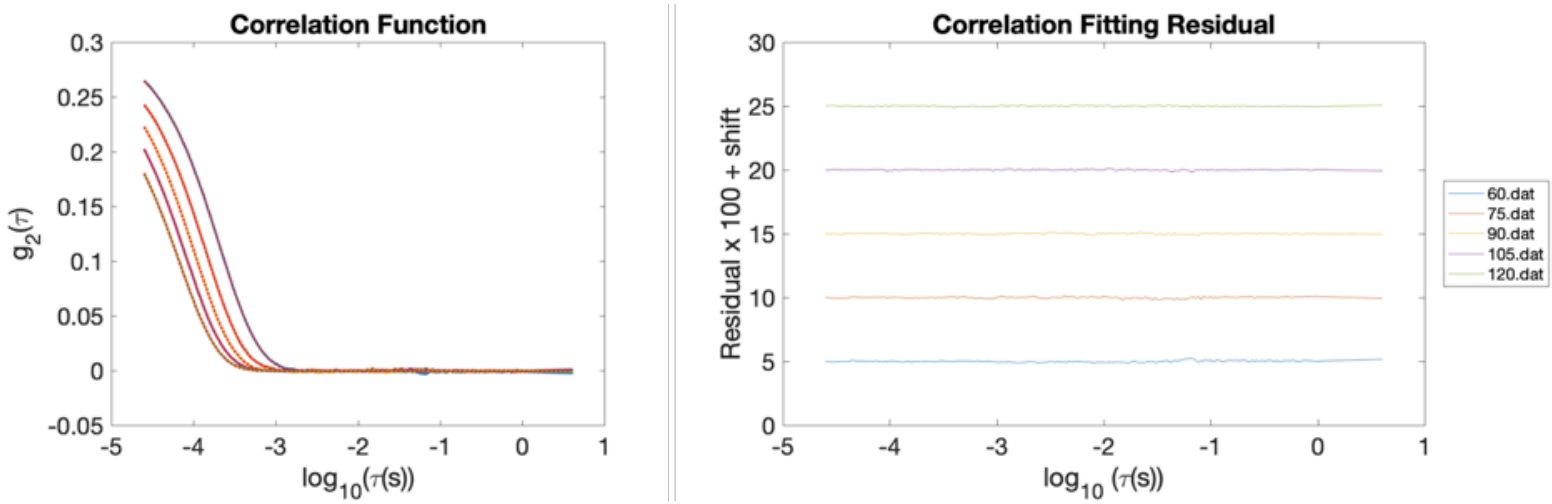

Figure S-10. Measured angular dependence of the autocorrelation function for $\mathrm{PEG}_{5 \mathrm{~K}}-\mathrm{PLK}_{47} /$ $\mathrm{PAA}_{50}$ at $0 \mathrm{mM} \mathrm{NaCl}$ between $60^{\circ}$ and $120^{\circ}$ fitted by cumulant expansion. 


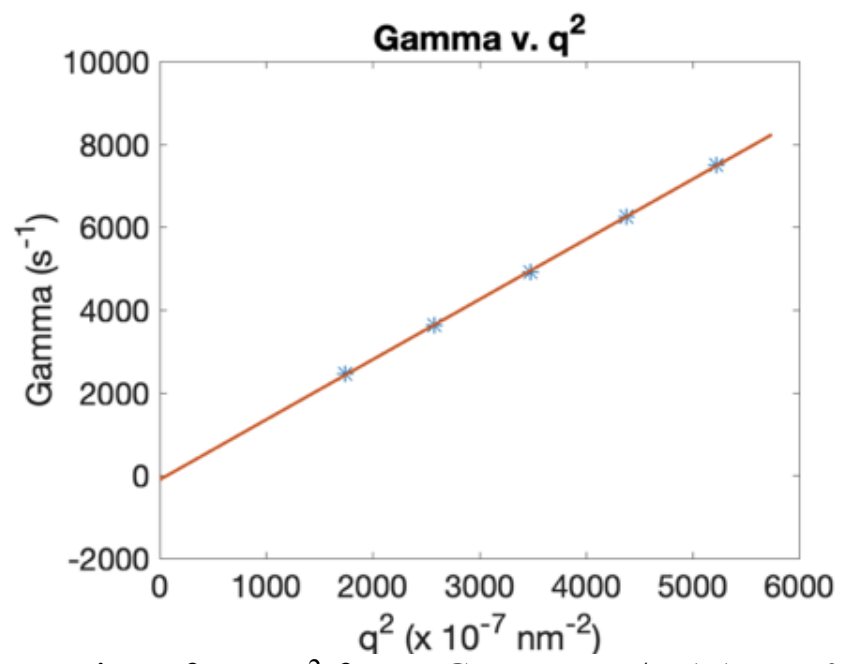

Figure S-11. Linear regression of $\Gamma$ vs $q^{2}$ for $\mathrm{PEG}_{5 \mathrm{~K}}-\mathrm{PLK}_{47} / \mathrm{PAA}_{50}$ at $0 \mathrm{mM} \mathrm{NaCl}$ over 5 angles between $60^{\circ}$ and $120^{\circ}$.
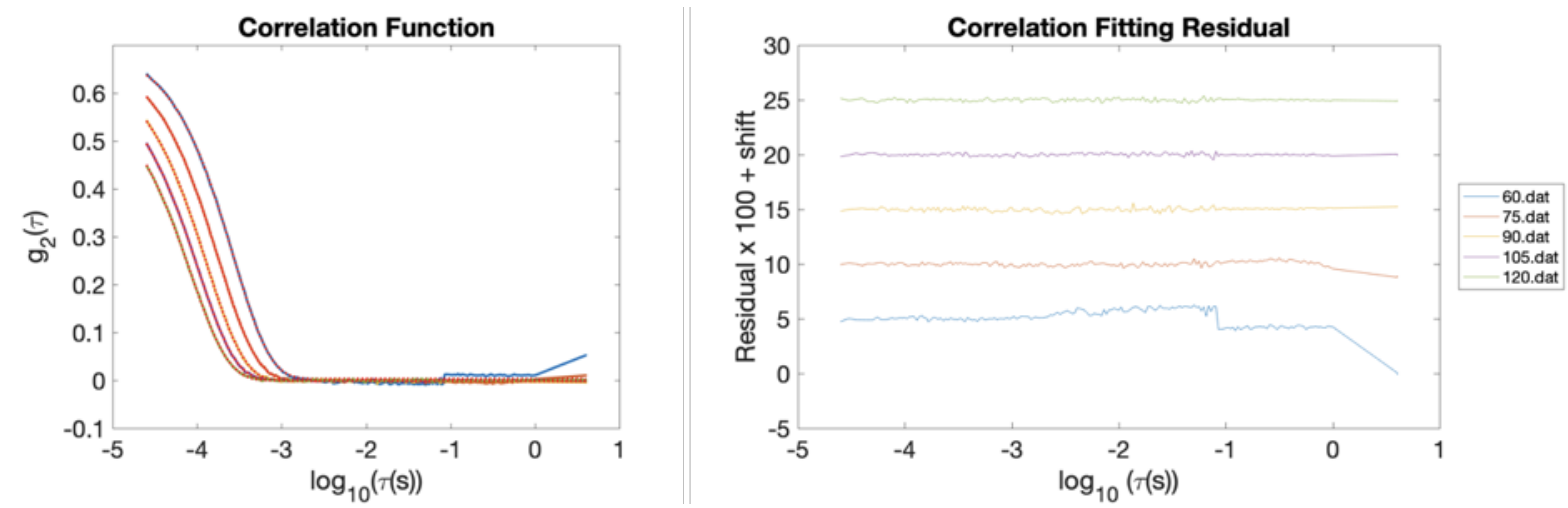

Figure S-12. Measured angular dependence of the autocorrelation function for $\mathrm{PEG}_{5 \mathrm{~K}}-\mathrm{PLK}_{47} /$ $\mathrm{PAA}_{50}$ at $100 \mathrm{mM} \mathrm{NaCl}$ between $60^{\circ}$ and $120^{\circ}$ fitted by cumulant expansion. 


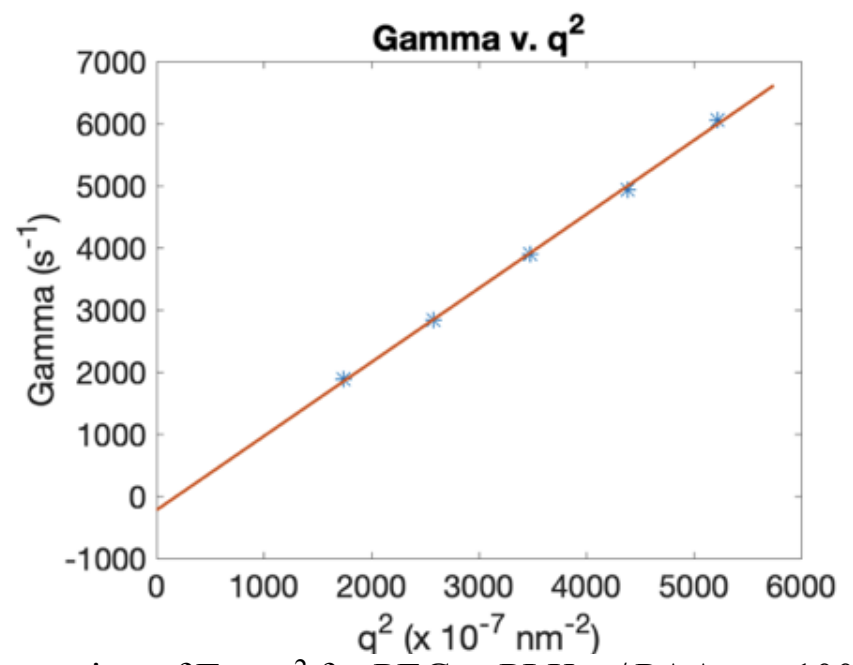

Figure S-13. Linear regression of $\Gamma$ vs $q^{2}$ for $\mathrm{PEG}_{5 \mathrm{~K}}-\mathrm{PLK}_{47} / \mathrm{PAA}_{50}$ at $100 \mathrm{mM} \mathrm{NaCl}$ over 5 angles between $60^{\circ}$ and $120^{\circ}$.
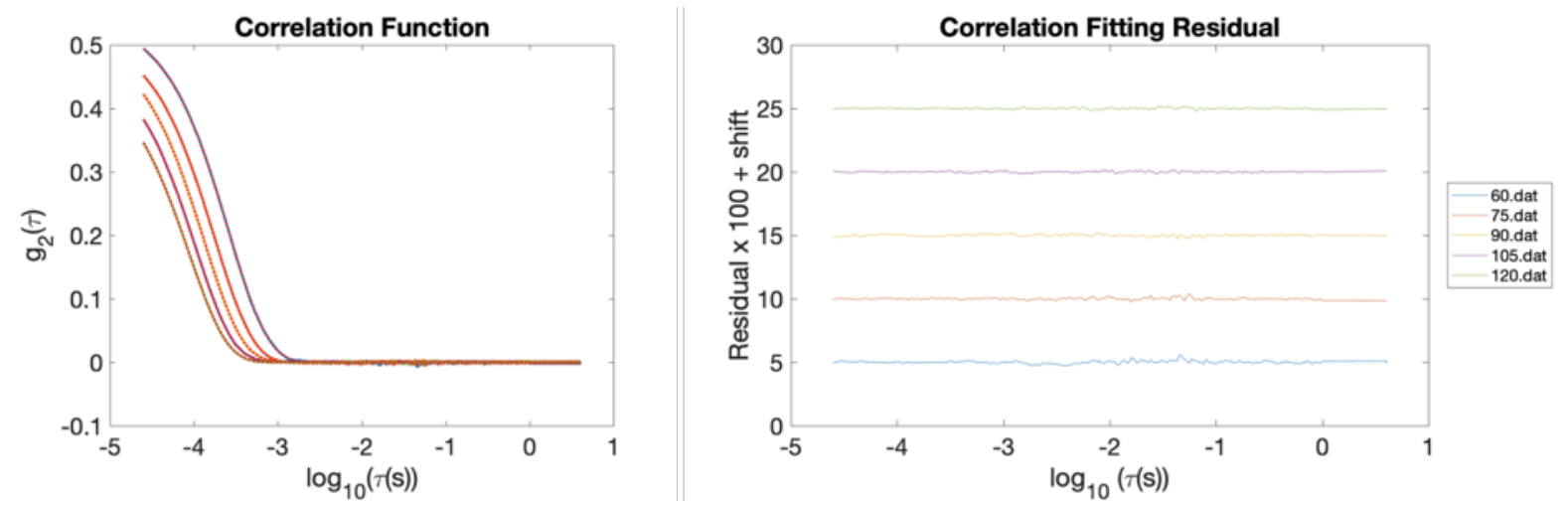

Figure S-14. Measured angular dependence of the autocorrelation function for $\mathrm{PEG}_{10 \mathrm{~K}}-\mathrm{PLK}_{93} /$ $\mathrm{PAA}_{50}$ at $0 \mathrm{mM} \mathrm{NaCl}$ between $60^{\circ}$ and $120^{\circ}$ fitted by cumulant expansion. 


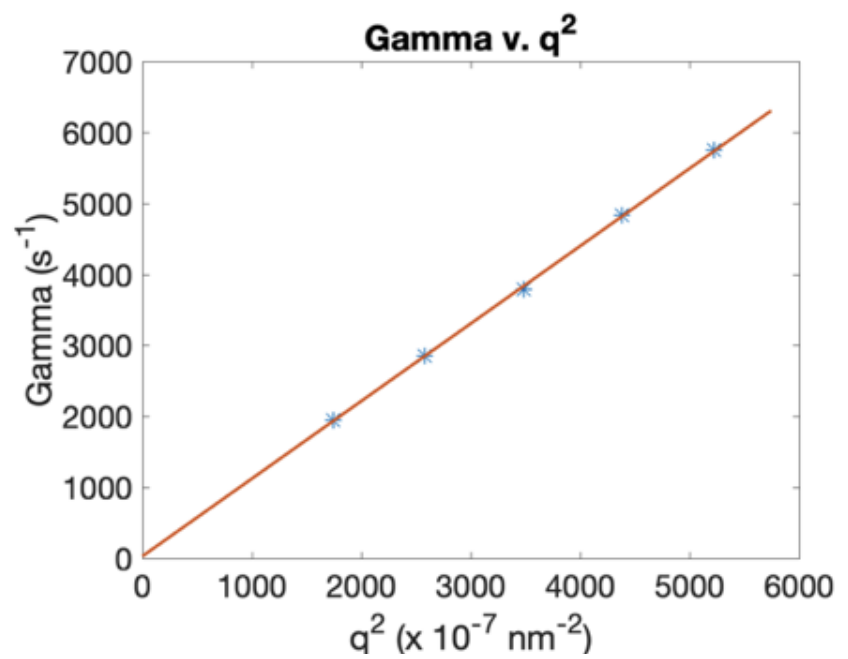

Figure S-15. Linear regression of $\Gamma$ vs $q^{2}$ for $\mathrm{PEG}_{10 \mathrm{~K}}-\mathrm{PLK}_{93} / \mathrm{PAA}_{50}$ at $0 \mathrm{mM} \mathrm{NaCl}$ over 5 angles between $60^{\circ}$ and $120^{\circ}$.
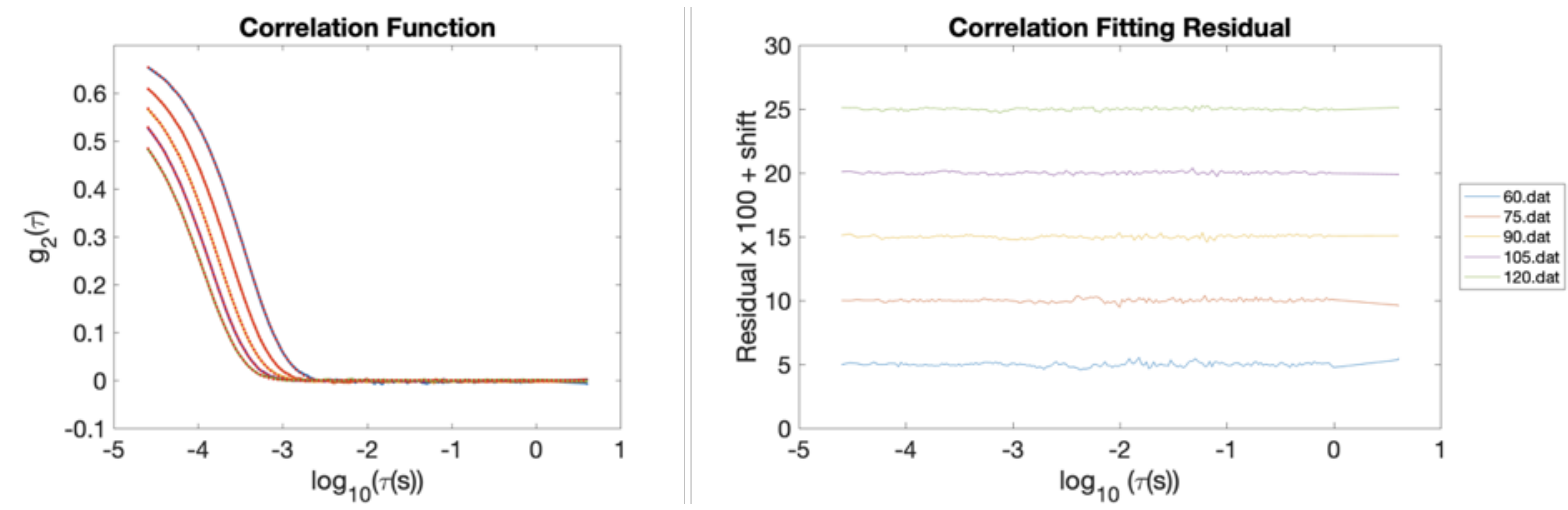

Figure S-16. Measured angular dependence of the autocorrelation function for $\mathrm{PEG}_{10 \mathrm{~K}}-\mathrm{PLK}_{93} /$ $\mathrm{PAA}_{50}$ at $100 \mathrm{mM} \mathrm{NaCl}$ between $60^{\circ}$ and $120^{\circ}$ fitted by cumulant expansion. 


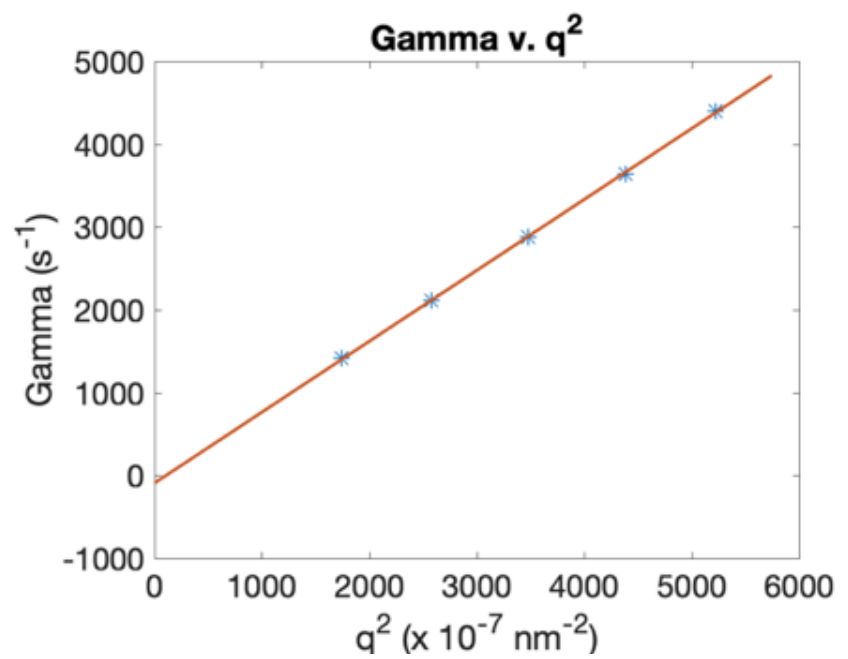

Figure S-17. Linear regression of $\Gamma$ vs $q^{2}$ for $\mathrm{PEG}_{10 \mathrm{~K}}-\mathrm{PLK}_{93} / \mathrm{PAA}_{50}$ at $100 \mathrm{mM} \mathrm{NaCl}$ over 5 angles between $60^{\circ}$ and $120^{\circ}$.
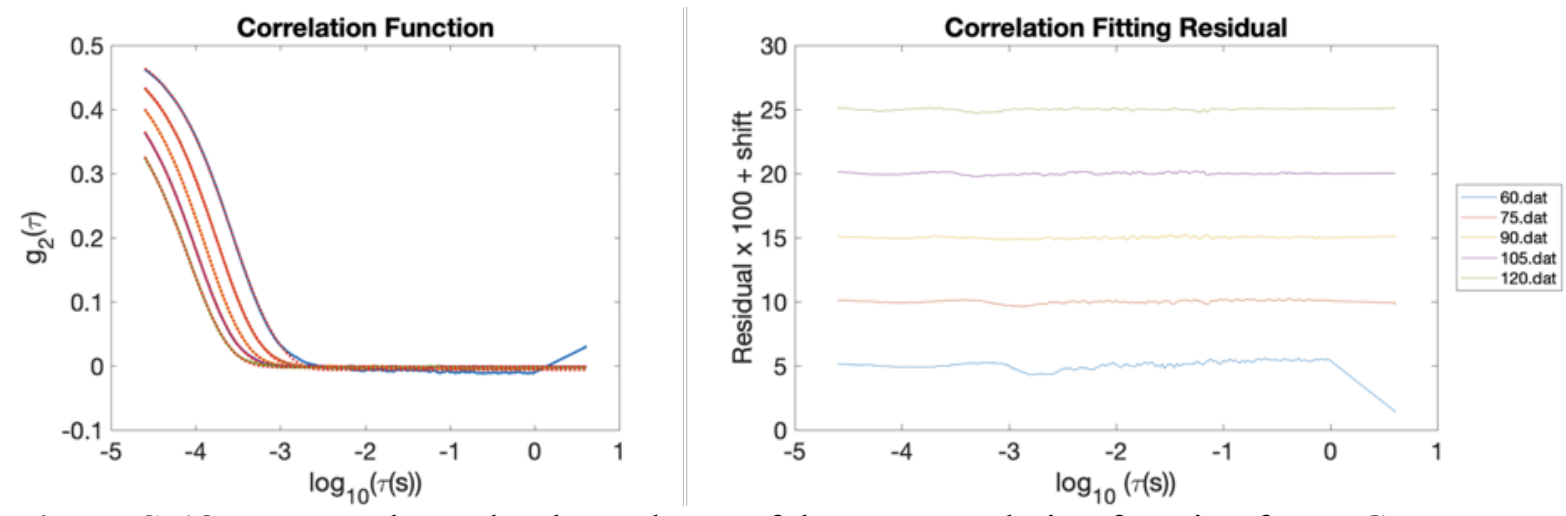

Figure S-18. Measured angular dependence of the autocorrelation function for PEG $_{5 \mathrm{~K}}-\mathrm{PVBTMA}_{53}$ / PAA 50 at $0 \mathrm{mM} \mathrm{NaCl}$ between $60^{\circ}$ and $120^{\circ}$ fitted by cumulant expansion. 


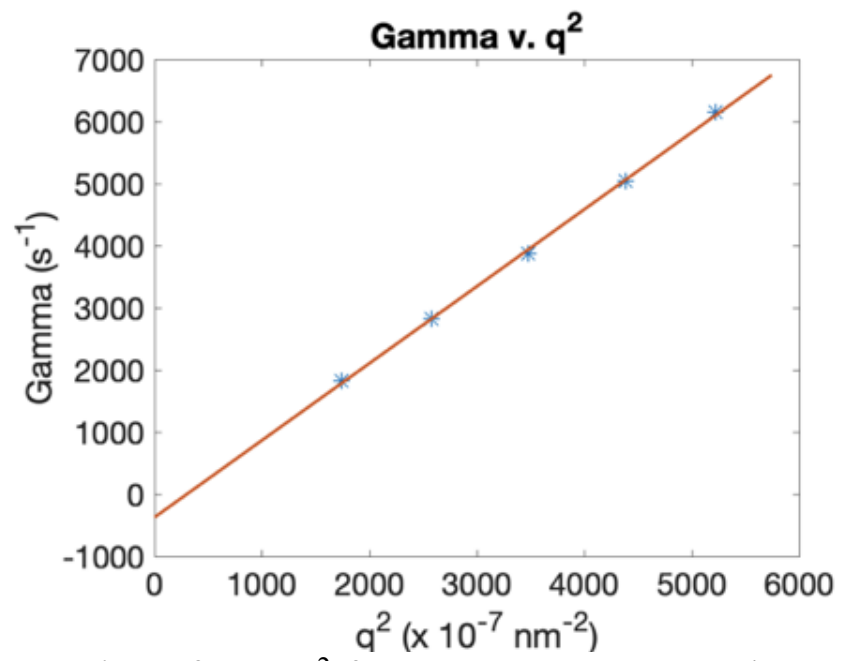

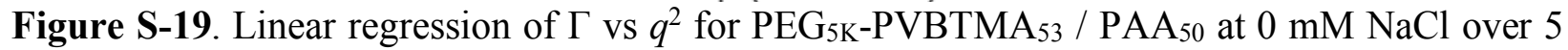
angles between $60^{\circ}$ and $120^{\circ}$.
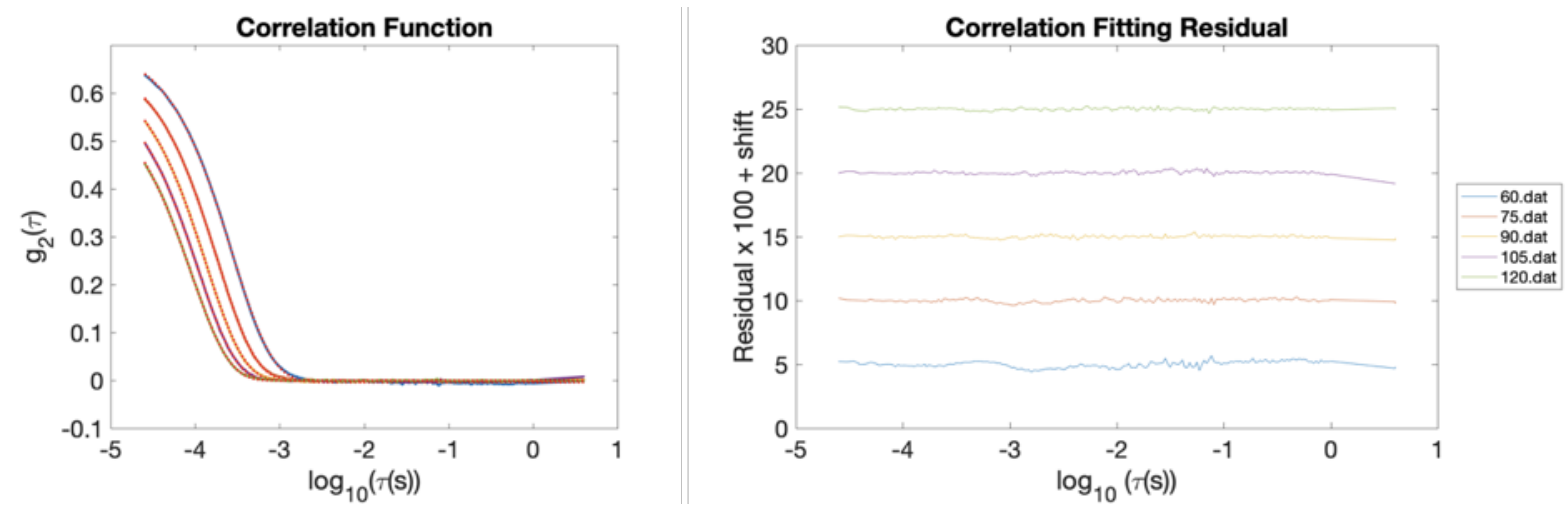

Figure S-20. Measured angular dependence of the autocorrelation function for $\mathrm{PEG}_{5 \mathrm{~K}}-\mathrm{PVBTMA}_{53}$ / $\mathrm{PAA}_{50}$ at $100 \mathrm{mM} \mathrm{NaCl}$ between $60^{\circ}$ and $120^{\circ}$ fitted by cumulant expansion. 


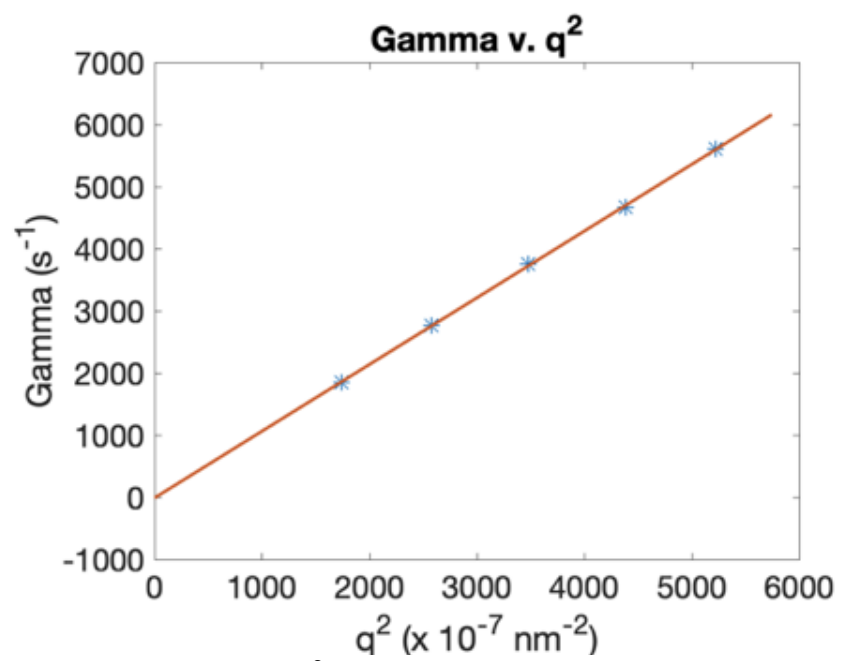

Figure S-21. Linear regression of $\Gamma$ vs $q^{2}$ for $\mathrm{PEG}_{5 \mathrm{~K}}-\mathrm{PVBTMA}_{53}$ at $100 \mathrm{mM} \mathrm{NaCl}$ over 5 angles between $60^{\circ}$ and $120^{\circ}$.
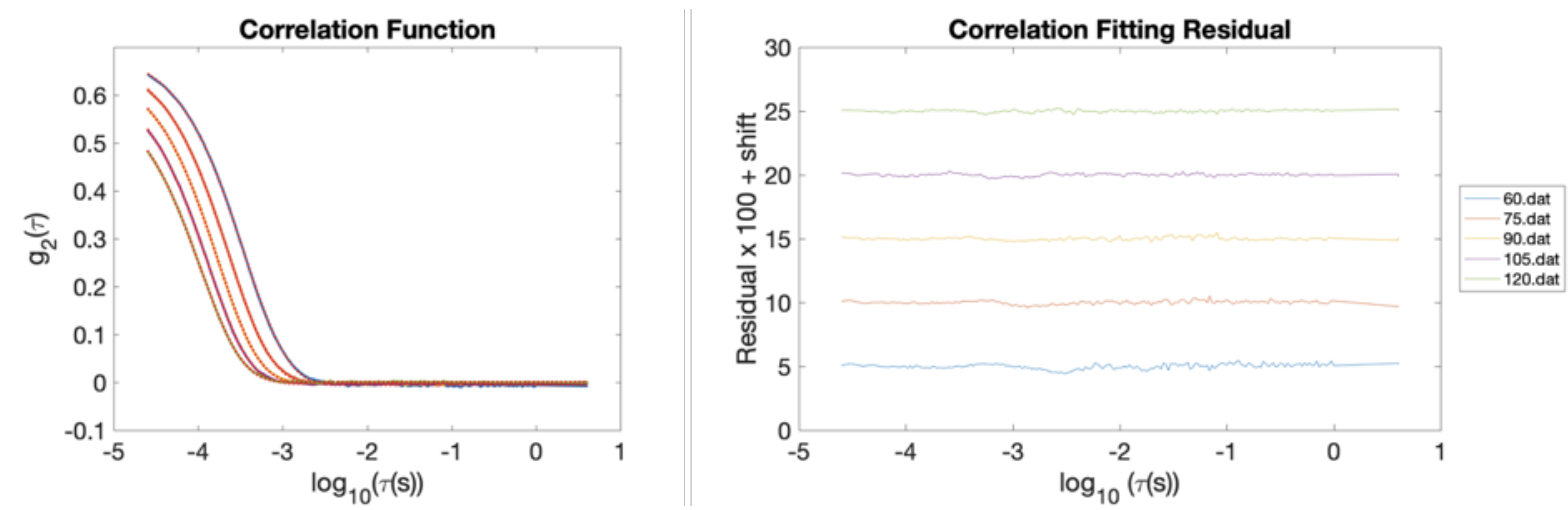

Figure S-22. Measured angular dependence of the autocorrelation function for $\mathrm{PEG}_{10 \mathrm{~K}-}$ $\mathrm{PVBTMA}_{100} / \mathrm{PAA}_{50}$ at $0 \mathrm{mM} \mathrm{NaCl}$ between $60^{\circ}$ and $120^{\circ}$ fitted by cumulant expansion. 


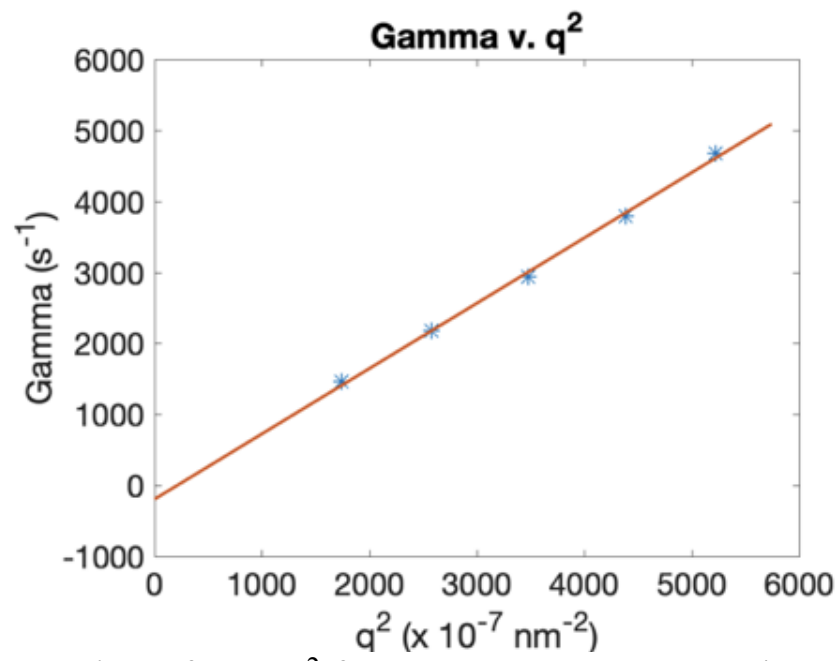

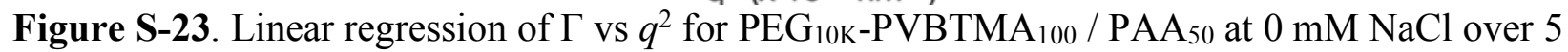
angles between $60^{\circ}$ and $120^{\circ}$.
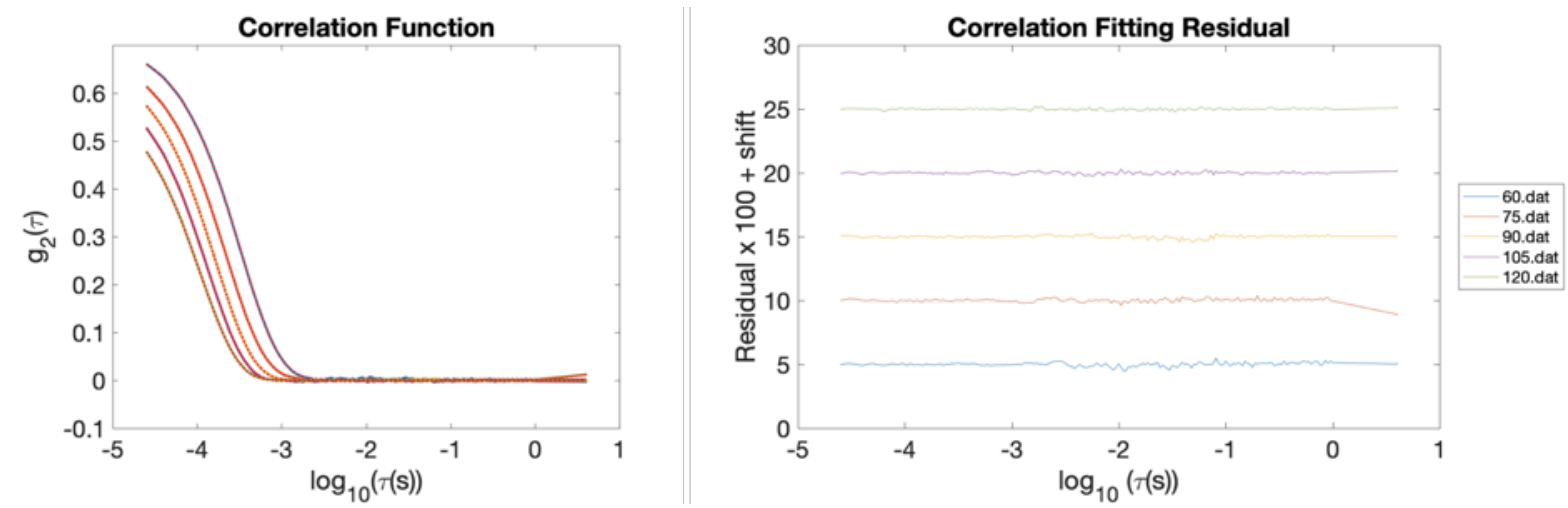

Figure S-24. Measured angular dependence of the autocorrelation function for $\mathrm{PEG}_{10 \mathrm{~K}}$ PVBTMA $_{100} / \mathrm{PAA}_{50}$ at $100 \mathrm{mM} \mathrm{NaCl}$ between $60^{\circ}$ and $120^{\circ}$ fitted by cumulant expansion. 


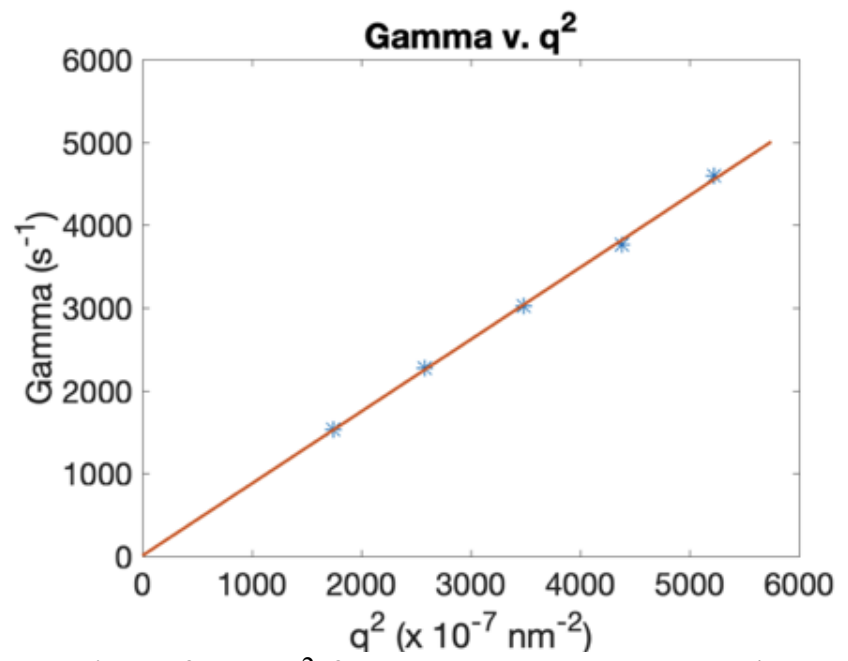

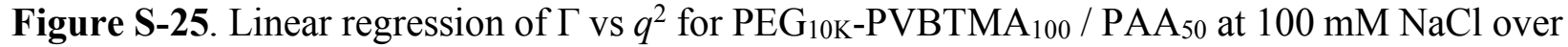
5 angles between $60^{\circ}$ and $120^{\circ}$.
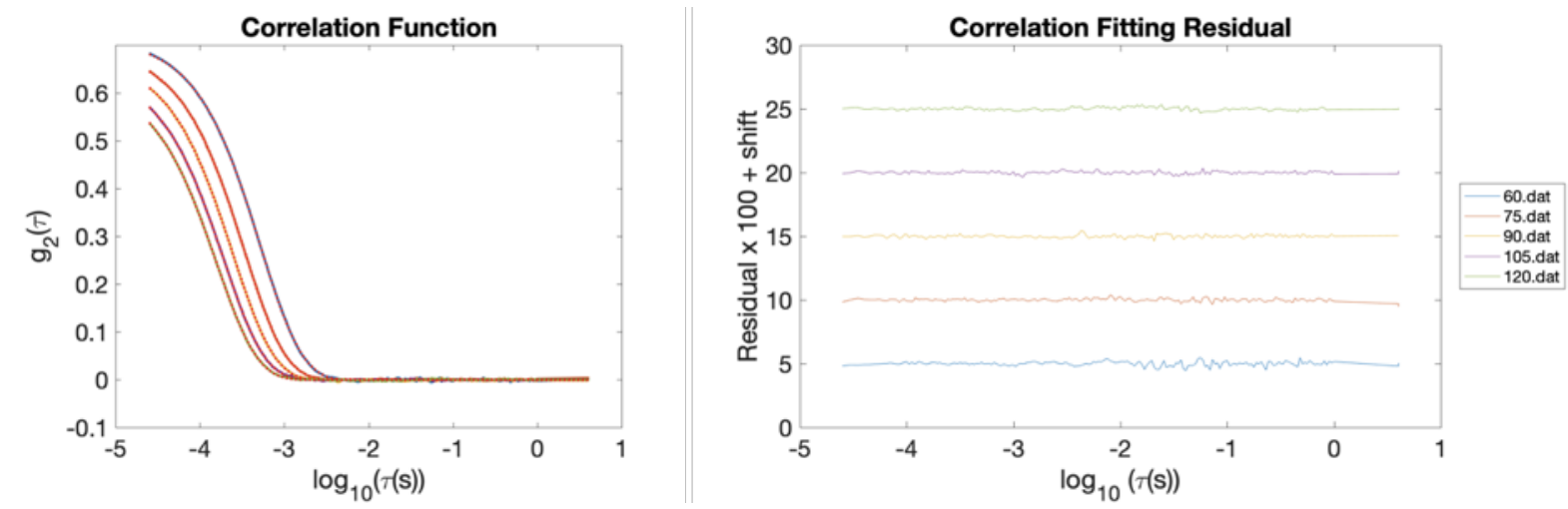

Figure S-26. Measured angular dependence of the autocorrelation function for $\mathrm{PMPC}_{5 \mathrm{~K}-}$ PVBTMA $_{50} / \mathrm{PAA}_{50}$ at $0 \mathrm{mM} \mathrm{NaCl}$ between $60^{\circ}$ and $120^{\circ}$ fitted by cumulant expansion. 


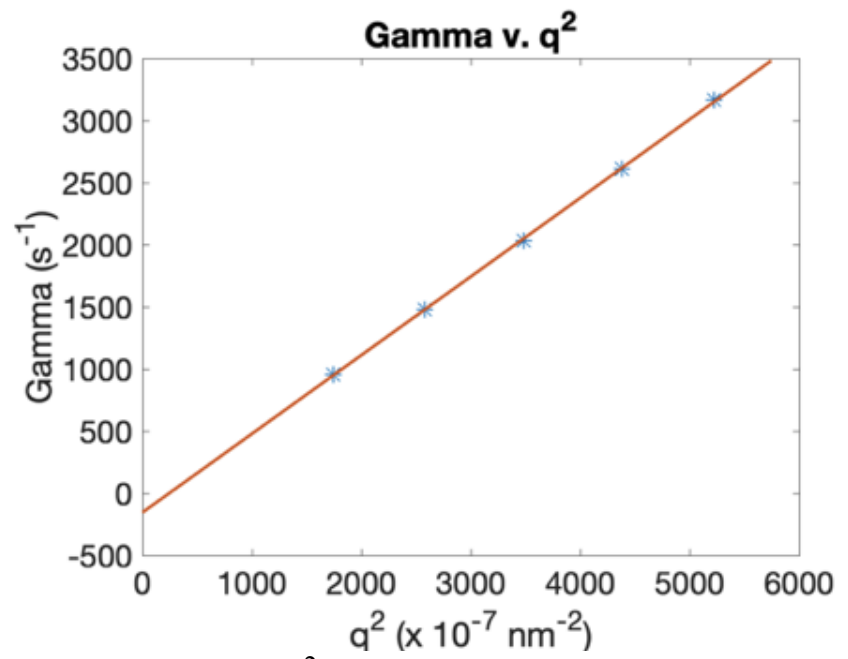

Figure S-27. Linear regression of $\Gamma$ vs $q^{2}$ for $\mathrm{PMPC}_{5 \mathrm{~K}}-\mathrm{PVBTMA}_{50} / \mathrm{PAA}_{50}$ at $0 \mathrm{mM} \mathrm{NaCl}$ over 5 angles between $60^{\circ}$ and $120^{\circ}$.
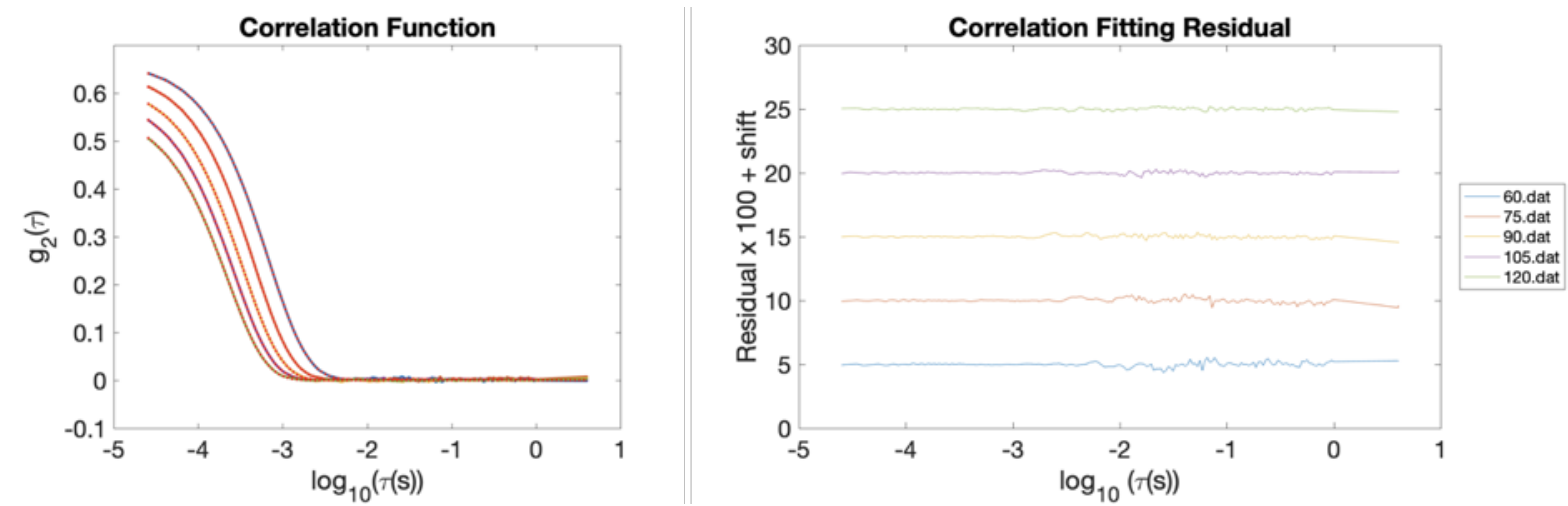

Figure S-28. Measured angular dependence of the autocorrelation function for $\mathrm{PMPC}_{5 \mathrm{~K}-}$ PVBTMA $_{50} / \mathrm{PAA}_{50}$ at $100 \mathrm{mM} \mathrm{NaCl}$ between $60^{\circ}$ and $120^{\circ}$ fitted by cumulant expansion. 


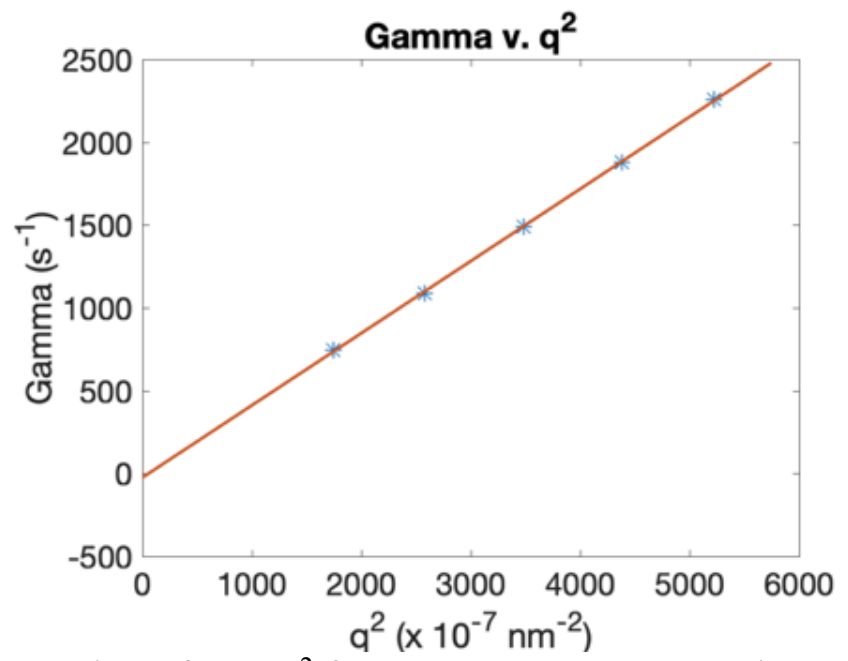

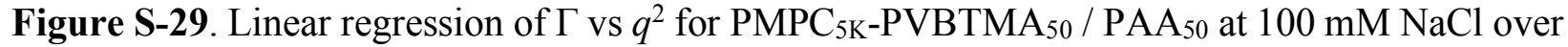
5 angles between $60^{\circ}$ and $120^{\circ}$.
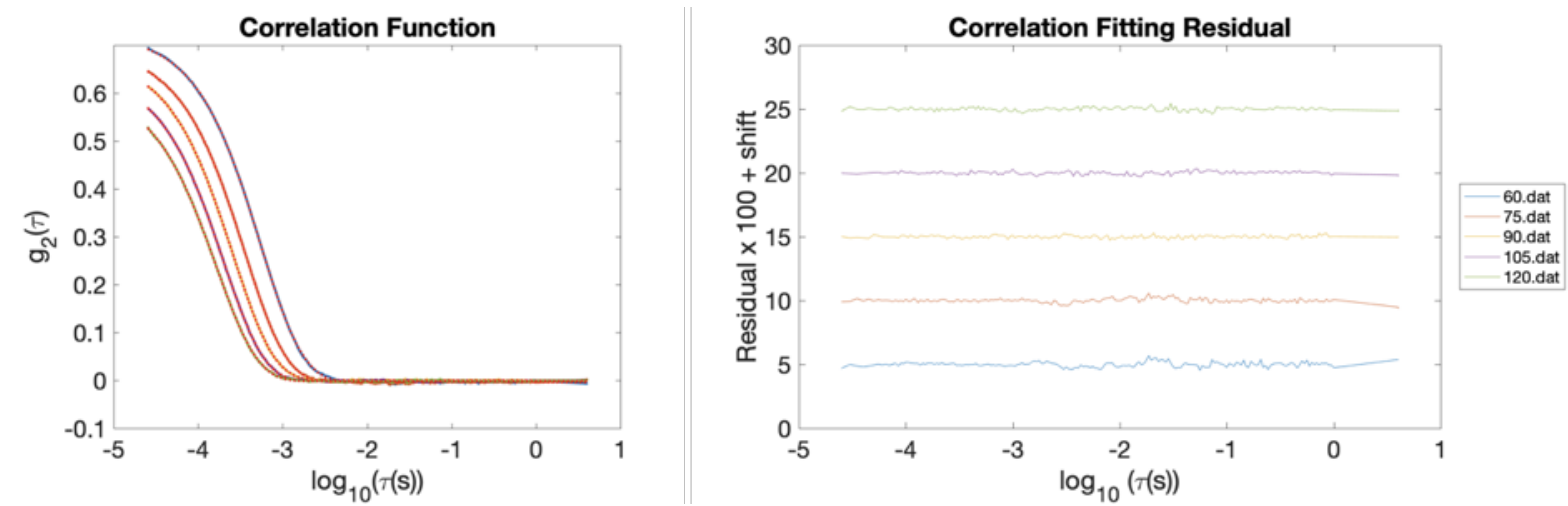

Figure S-30. Measured angular dependence of the autocorrelation function for $\mathrm{PMPC}_{10 \mathrm{~K}}-$ PVBTMA $_{97} / \mathrm{PAA}_{50}$ at $0 \mathrm{mM} \mathrm{NaCl}$ between $60^{\circ}$ and $120^{\circ}$ fitted by cumulant expansion. 


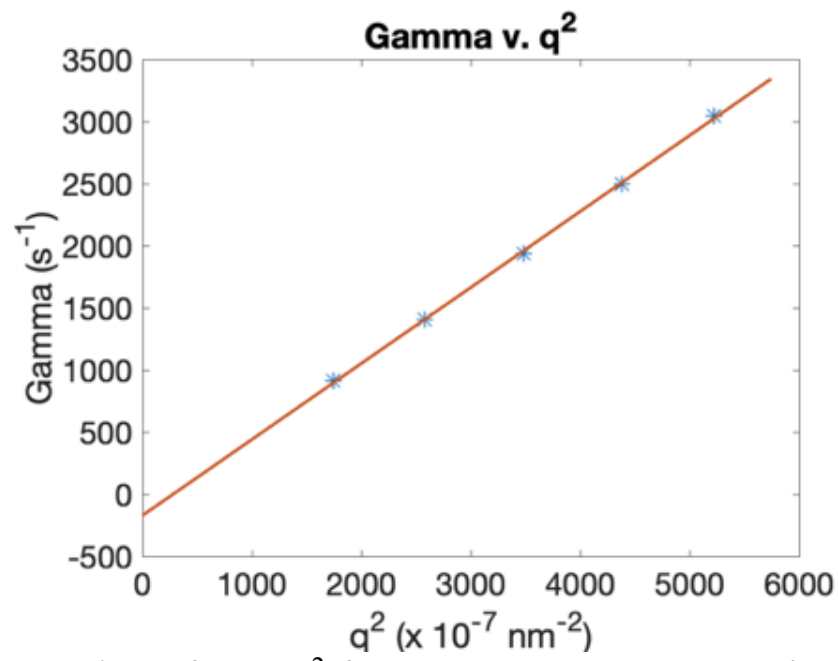

Figure S-31. Linear regression of $\Gamma$ vs $q^{2}$ for $\mathrm{PMPC}_{10 \mathrm{~K}}-\mathrm{PVBTMA}_{97} / \mathrm{PAA}_{50}$ at $0 \mathrm{mM} \mathrm{NaCl}$ over 5 angles between $60^{\circ}$ and $120^{\circ}$.
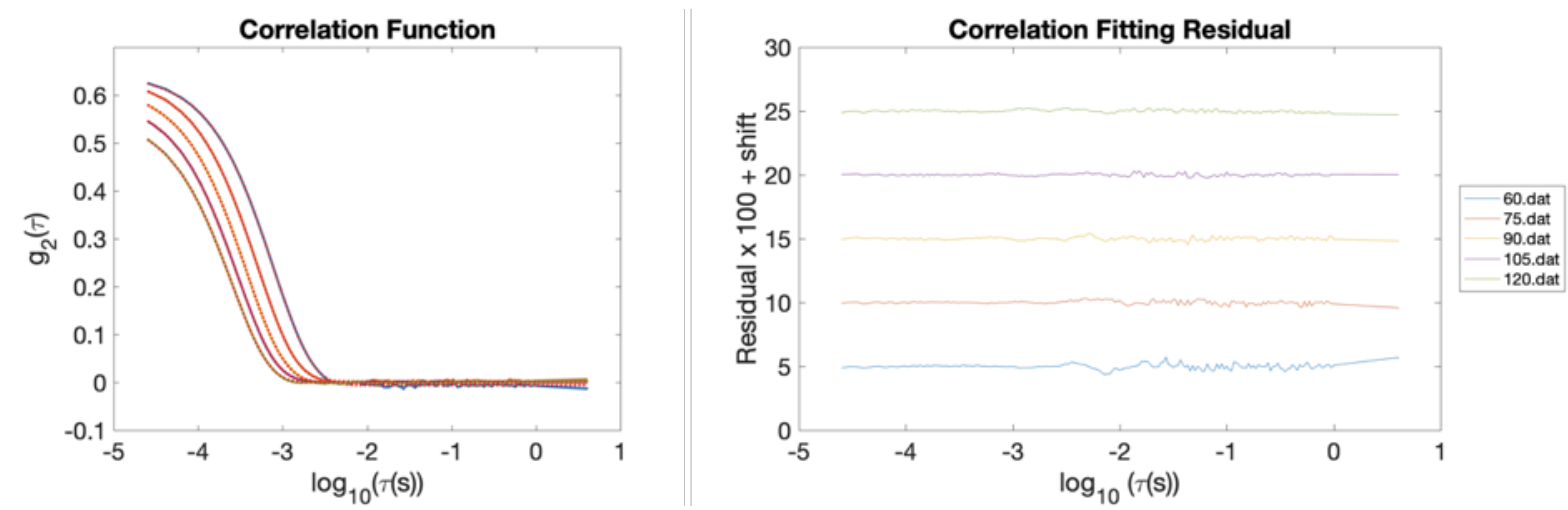

Figure S-32. Measured angular dependence of the autocorrelation function for $\mathrm{PMPC}_{10 \mathrm{~K}-}$ $\mathrm{PVBTMA}_{97} / \mathrm{PAA}_{50}$ at $100 \mathrm{mM} \mathrm{NaCl}$ between $60^{\circ}$ and $120^{\circ}$ fitted by cumulant expansion. 


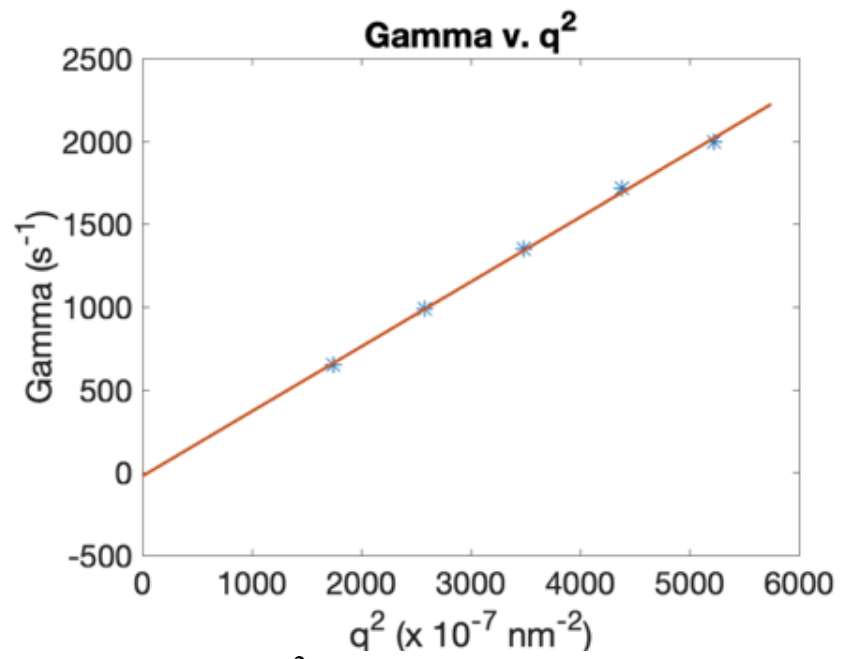

Figure S-33. Linear regression of $\Gamma$ vs $q^{2}$ for $\mathrm{PMPC}_{10 \mathrm{~K}}-\mathrm{PVBTMA}_{97} / \mathrm{PAA}_{50}$ at $100 \mathrm{mM} \mathrm{NaCl}$ over 5 angles between $60^{\circ}$ and $120^{\circ}$. 
Figures S-34 through S-37 show the autocorrelation functions that correspond to Figure S-9 for all investigated micelle systems from 0 to $200 \mathrm{mM} \mathrm{NaCl}$.
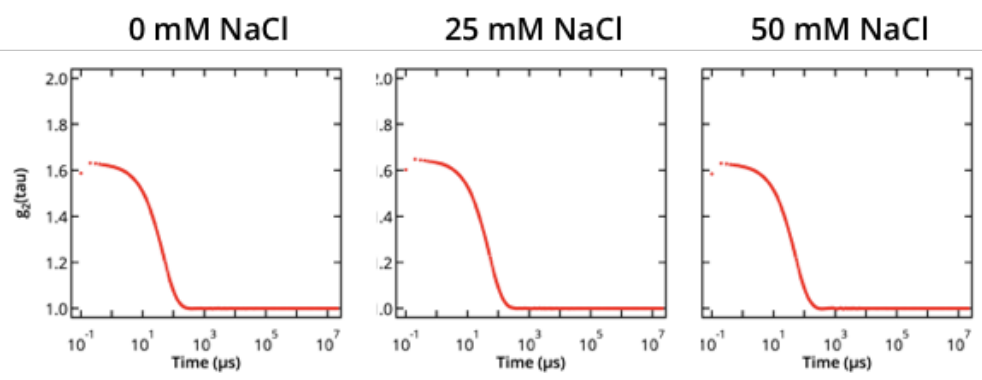

$100 \mathrm{mM} \mathrm{NaCl}$ $200 \mathrm{mM} \mathrm{NaCl}$
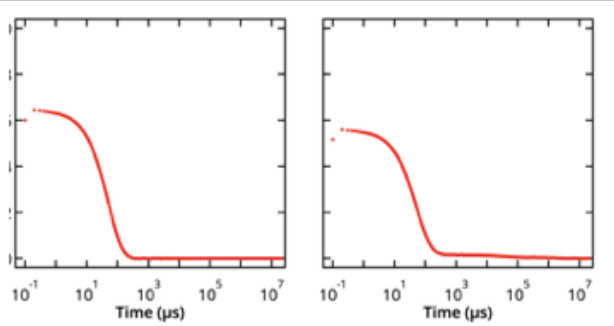

Figure S-34. Summary of the autocorrelation functions of PCM assemblies $\mathrm{PEG}_{5 \mathrm{~K}}-\mathrm{PVBTMA}_{53}$ at (A) $0 \mathrm{mM}$, (B) $25 \mathrm{mM}$, (C) $50 \mathrm{mM}$, (D) $100 \mathrm{mM}$, and (E) $200 \mathrm{mM} \mathrm{NaCl}$.
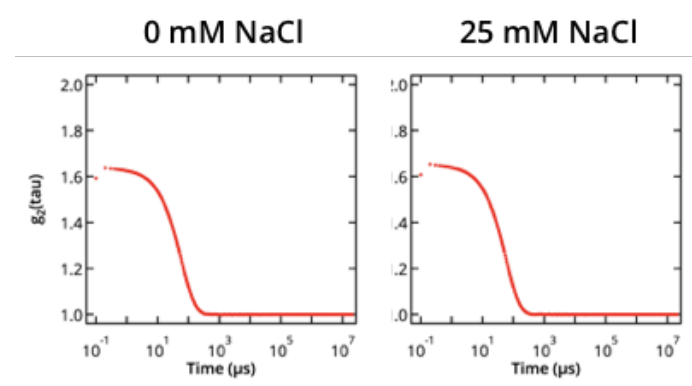

\section{$50 \mathrm{mM} \mathrm{NaCl}$}

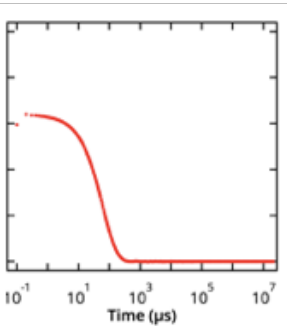

$100 \mathrm{mM} \mathrm{NaCl}$

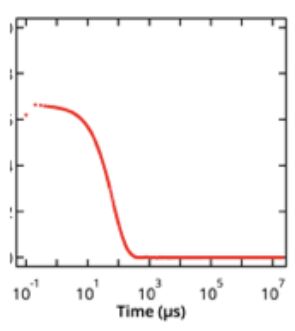

$200 \mathrm{mM} \mathrm{NaCl}$

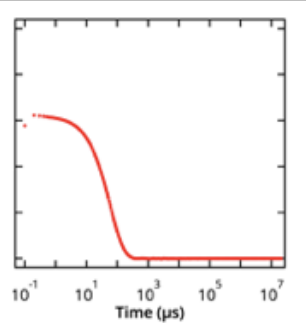

Figure S-35. Summary of the autocorrelation functions of PCM assemblies $\mathrm{PEG}_{10 \mathrm{~K}}-\mathrm{PVBTMA}_{100}$ at (A) $0 \mathrm{mM}$, (B) $25 \mathrm{mM}$, (C) $50 \mathrm{mM}$, (D) $100 \mathrm{mM}$, and (E) $200 \mathrm{mM} \mathrm{NaCl}$.
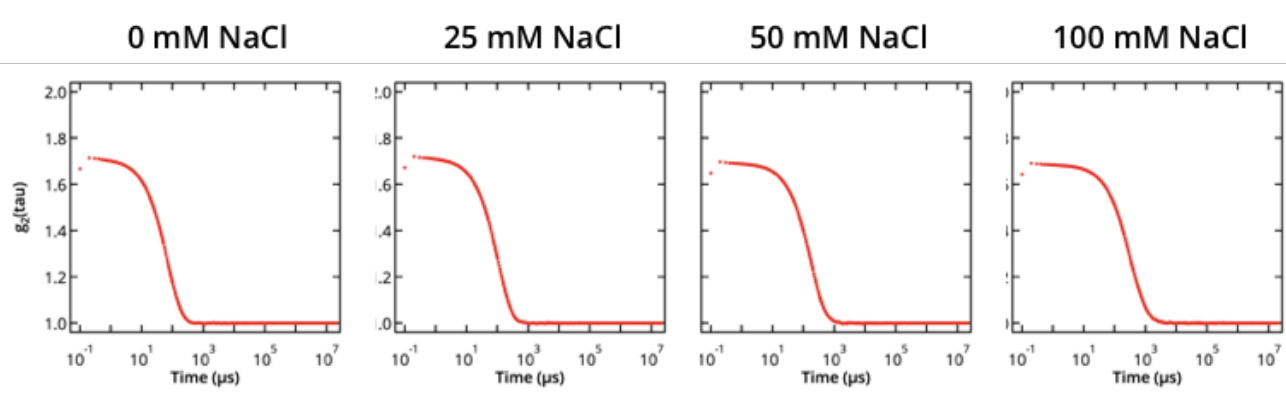

$200 \mathrm{mM} \mathrm{NaCl}$

Figure S-36. Summary of the autocorrelation functions of PCM assemblies PMPC $5 \mathrm{~K}-\mathrm{PVBTMA}_{50}$ at (A) $0 \mathrm{mM}$, (B) $25 \mathrm{mM}$, (C) $50 \mathrm{mM}$, (D) $100 \mathrm{mM}$, and (E) $200 \mathrm{mM} \mathrm{NaCl}$. 

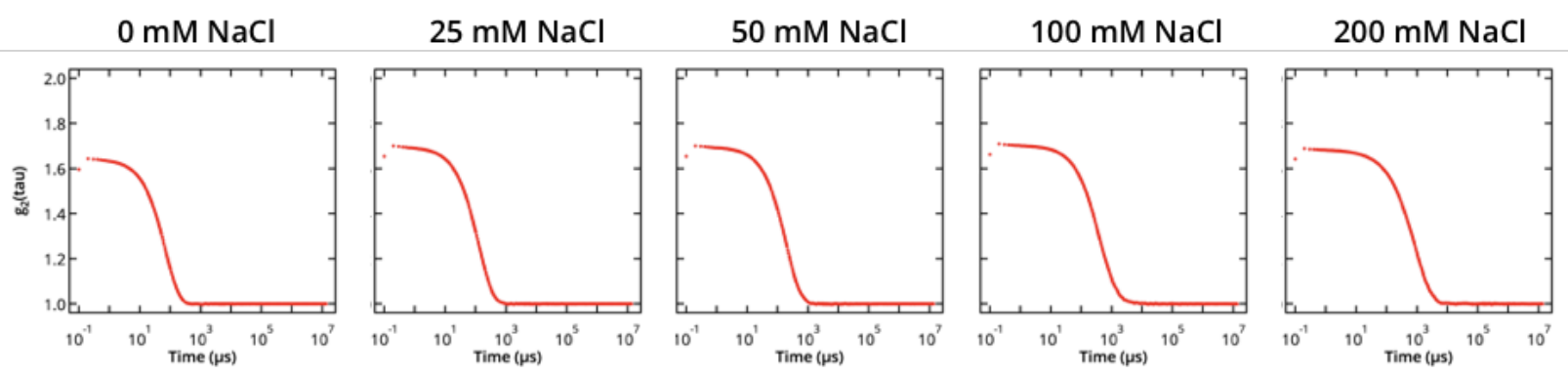

Figure S-37. Summary of the autocorrelation functions of PCM assemblies PMPC $10 \mathrm{~K}-\mathrm{PVBTMA}_{97}$ at (A) $0 \mathrm{mM}$, (B) $25 \mathrm{mM}$, (C) $50 \mathrm{mM}$, (D) $100 \mathrm{mM}$, and (E) $200 \mathrm{mM} \mathrm{NaCl}$.

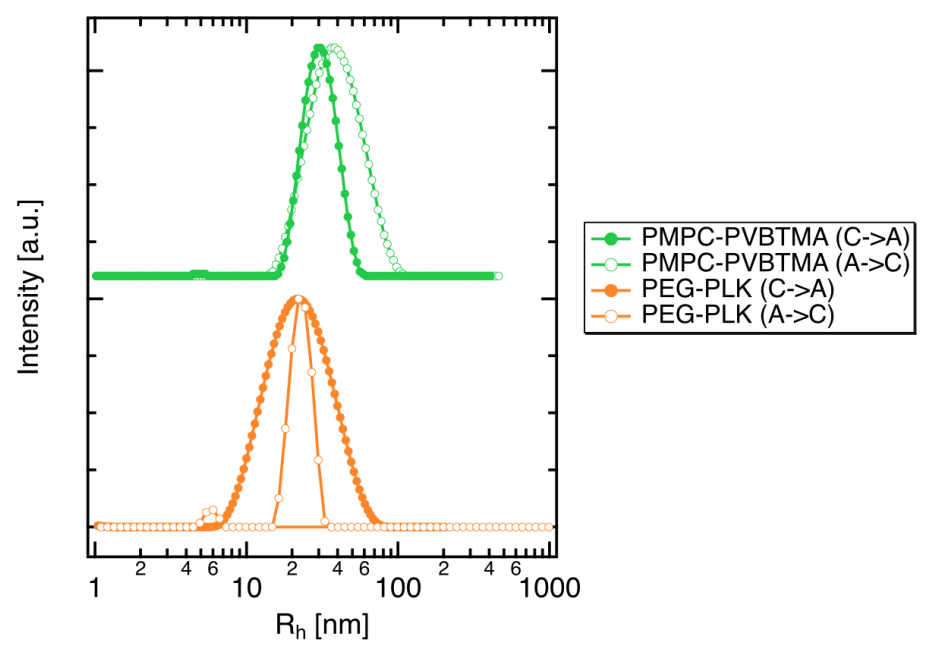

Figure S-38. REPES fits for DLS of PCMs assembled in different orders. All micelles in this work were assembled with the cation added to the solution first, followed by the anion $(C \rightarrow A)$. When this order is reversed $(\mathrm{A} \rightarrow \mathrm{C})$ the size distribution is different, suggesting that the system may be kinetically trapped. 


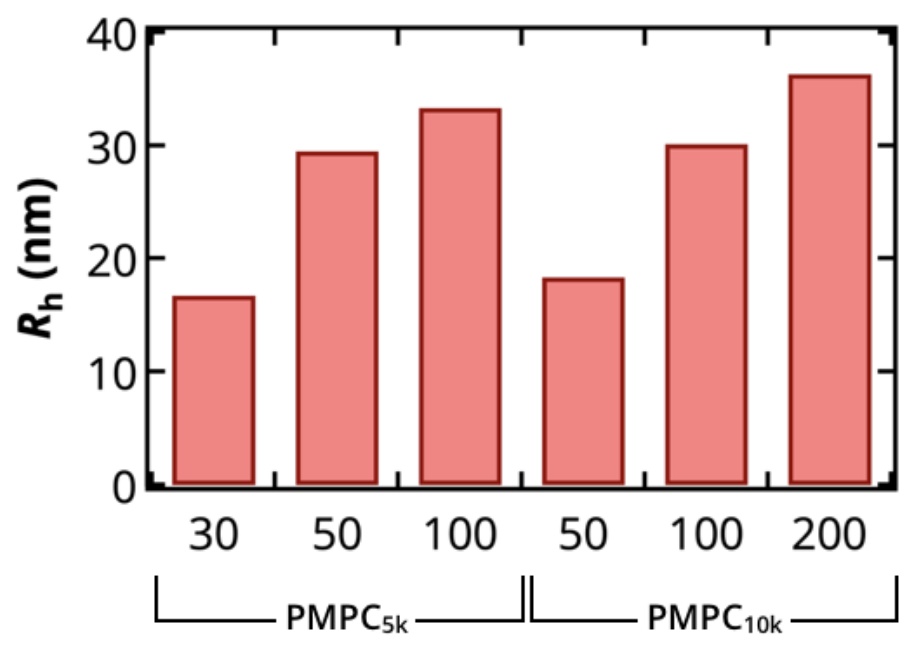

\section{PVBTMA Length}

Figure S-39. Comparison of the apparent hydrodynamic radius versus charged block length of PVBTMA in PCM assemblies. 


\section{S3. Small-Angle X-Ray Scattering}

Figure S-40 shows the intensity versus q profiles of the PEG-PLK, PEG-PVBTMA, and PMPC-PVBTMA polyelectrolyte solutions with increasing $\mathrm{NaCl}$ salt. At $0 \mathrm{mM} \mathrm{NaCl}$, the correlation peak can be observed at $\mathrm{q}=0.4-0.5 \AA$ for PEG-PLK and $\mathrm{q}=0.2-0.4 \AA$ for PEGPVBTMA and PMPC-PVBTMA. Table S-2 contains the fitting for the SAXS data in Figure 7 of the main manuscript with added $\mathrm{MgCl}_{2}$ salt.
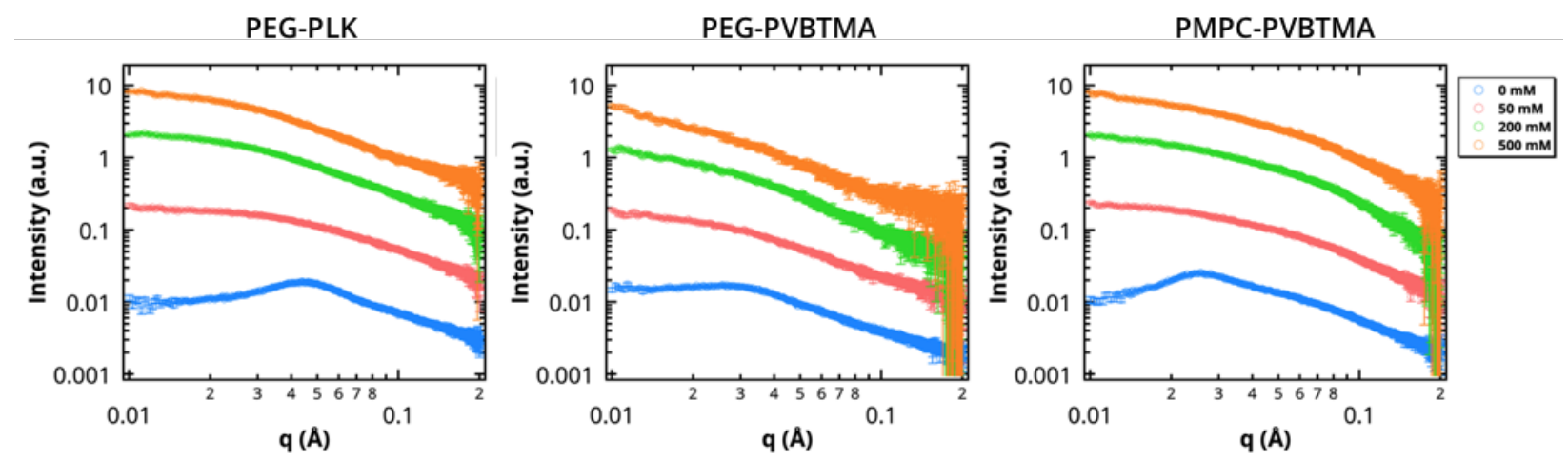

Figure S-40. SAXS profiles of polyelectrolyte solutions of PEG-PLK, PEG-PVBTMA, and PMPC-PVBTMA at polymer concentrations of $5 \mathrm{mg} / \mathrm{mL}$ with increasing added $\mathrm{NaCl}$ salt. Intensity scaled for clarity. 
Table S-2. SAXS summary with added $\mathrm{MgCl}_{2}$ salt.

\begin{tabular}{|c|c|c|c|}
\hline Sample & {$\left[\mathrm{MgCl}_{2}\right](\mathbf{m M})$} & $\mathbf{R}^{\mathrm{a}}(\mathbf{n m})$ & PDI $^{b}$ \\
\hline \multirow{2}{*}{$\mathrm{PEG}_{5 \mathrm{~K}}-\mathrm{PLK}_{47} / \mathrm{PAA}_{50}$} & 50 & 8.0 & 0.16 \\
\hline & 100 & 8.6 & 0.14 \\
\hline \multirow{2}{*}{$\mathrm{PEG}_{10 \mathrm{~K}}-\mathrm{PLK}_{93} / \mathrm{PAA}_{50}$} & 50 & 11.2 & 0.15 \\
\hline & 100 & 10.6 & 0.09 \\
\hline \multirow{2}{*}{$\mathrm{PEG}_{5 \mathrm{~K}}-\mathrm{PVBTMA}_{53} / \mathrm{PAA}_{50}$} & 50 & 10.7 & 0.09 \\
\hline & 100 & - & - \\
\hline \multirow{2}{*}{$\mathrm{PEG}_{10 \mathrm{~K}}-\mathrm{PVBTMA}_{100} / \mathrm{PAA}_{50}$} & 50 & 19.8 & 0.15 \\
\hline & 100 & - & - \\
\hline \multirow{2}{*}{$\mathrm{PMPC}_{5 \mathrm{~K}}-\mathrm{PVBTMA}_{50} / \mathrm{PAA}_{50}$} & 50 & - & - \\
\hline & 100 & - & - \\
\hline \multirow{2}{*}{$\mathrm{PMPC}_{10 \mathrm{~K}}-\mathrm{PVBTMA}_{97} / \mathrm{PAA}_{50}$} & 50 & - & - \\
\hline & 100 & - & - \\
\hline
\end{tabular}

${ }^{a}$ Mean core radius $(\mathrm{nm}){ }^{\mathrm{b}}$ Polydispersity index $\left(\sigma^{2} / \mathrm{R}^{2}\right)$. Models use Schulz-Zimm distribution flexible cylinder and Unified Level fits, except for PEG $\mathrm{PEK}_{10 \mathrm{~K}} \mathrm{PVBTMA}_{47} / \mathrm{PAA}_{100}$ (spheroid) and $\mathrm{PEG}_{10 \mathrm{~K}}-\mathrm{PLK}_{93} / \mathrm{PAA}_{50}$ (cylinder fitting).

For each polymer system, Figures S-40 through S-41 show the small angle X-ray scattering profile in water with 100,250 , and $500 \mathrm{mM} \mathrm{NaCl}$ and 50 and $100 \mathrm{mM} \mathrm{MgCl}_{2}$. 

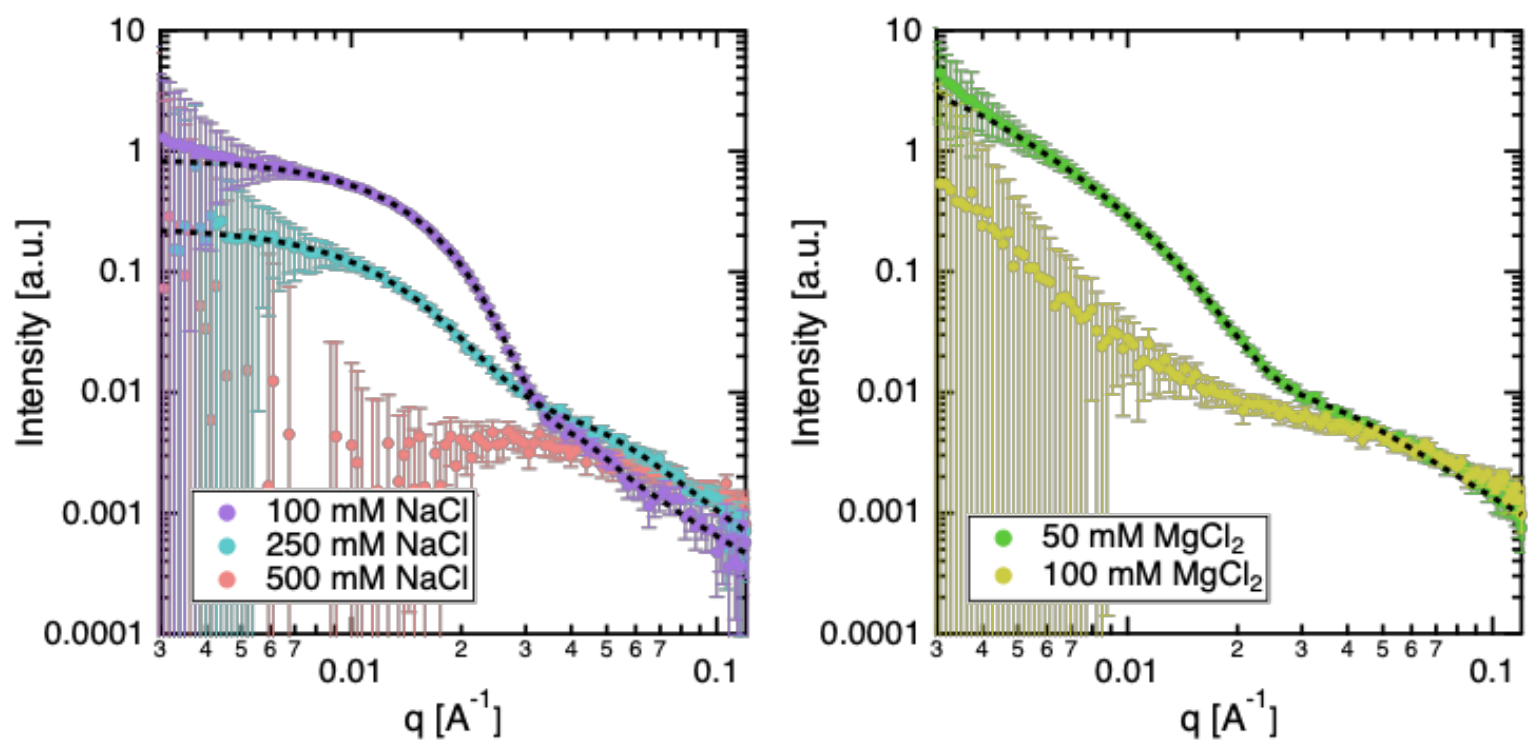

Figure S-40. SAXS profiles for $\mathrm{PEG}_{5 \mathrm{~K}}-\mathrm{PVBTMA}_{53} / \mathrm{PAA}_{50}$ with 100,250 , and $500 \mathrm{mM} \mathrm{NaCl}$ (left), as well as 50 and $100 \mathrm{mM} \mathrm{MgCl}_{2}$ (right).
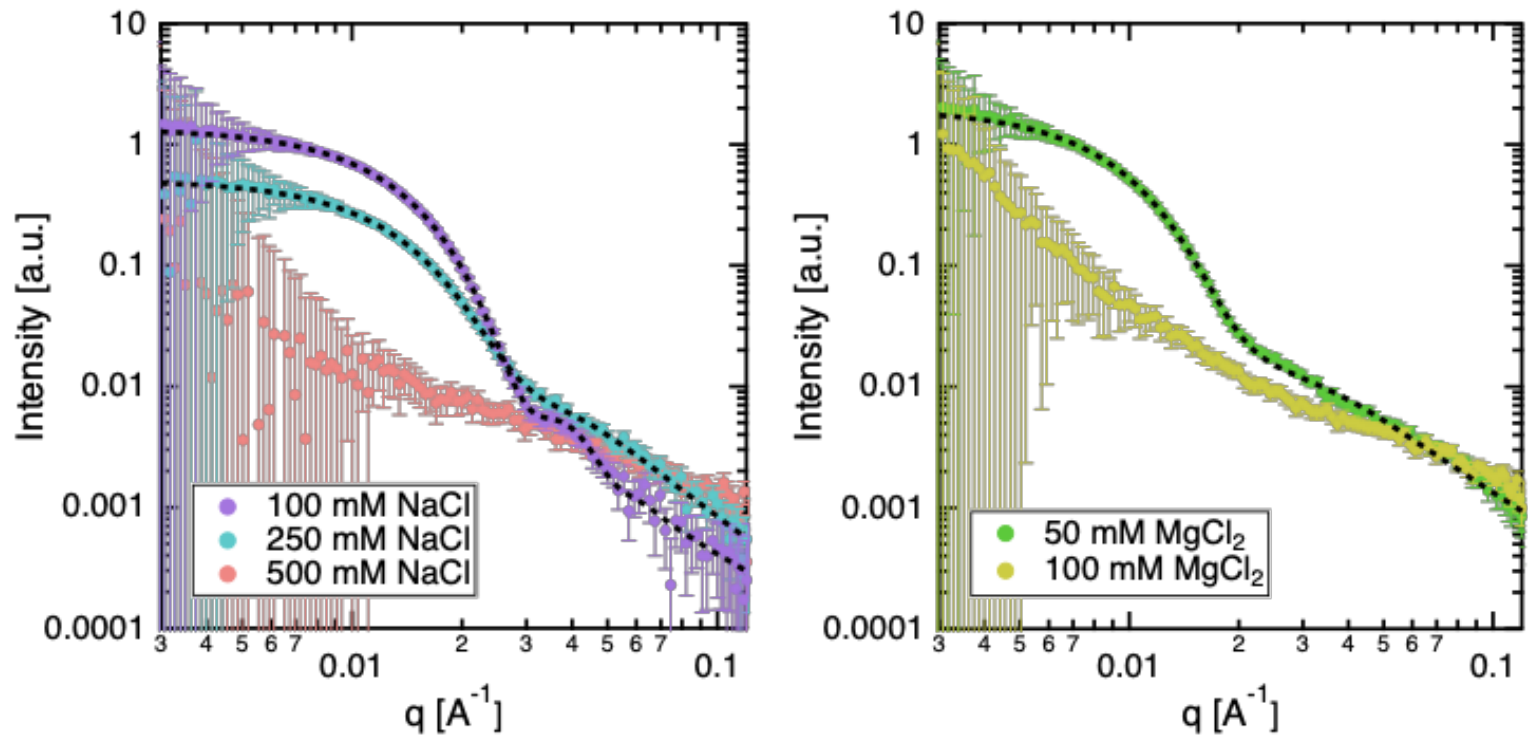

Figure S-41. SAXS profiles for $\mathrm{PEG}_{10 \mathrm{~K}}-\mathrm{PVBTMA}_{100} / \mathrm{PAA}_{50}$ with 100, 250, and $500 \mathrm{mM} \mathrm{NaCl}$ (left), as well as 50 and $100 \mathrm{mM} \mathrm{MgCl}_{2}$ (right). 

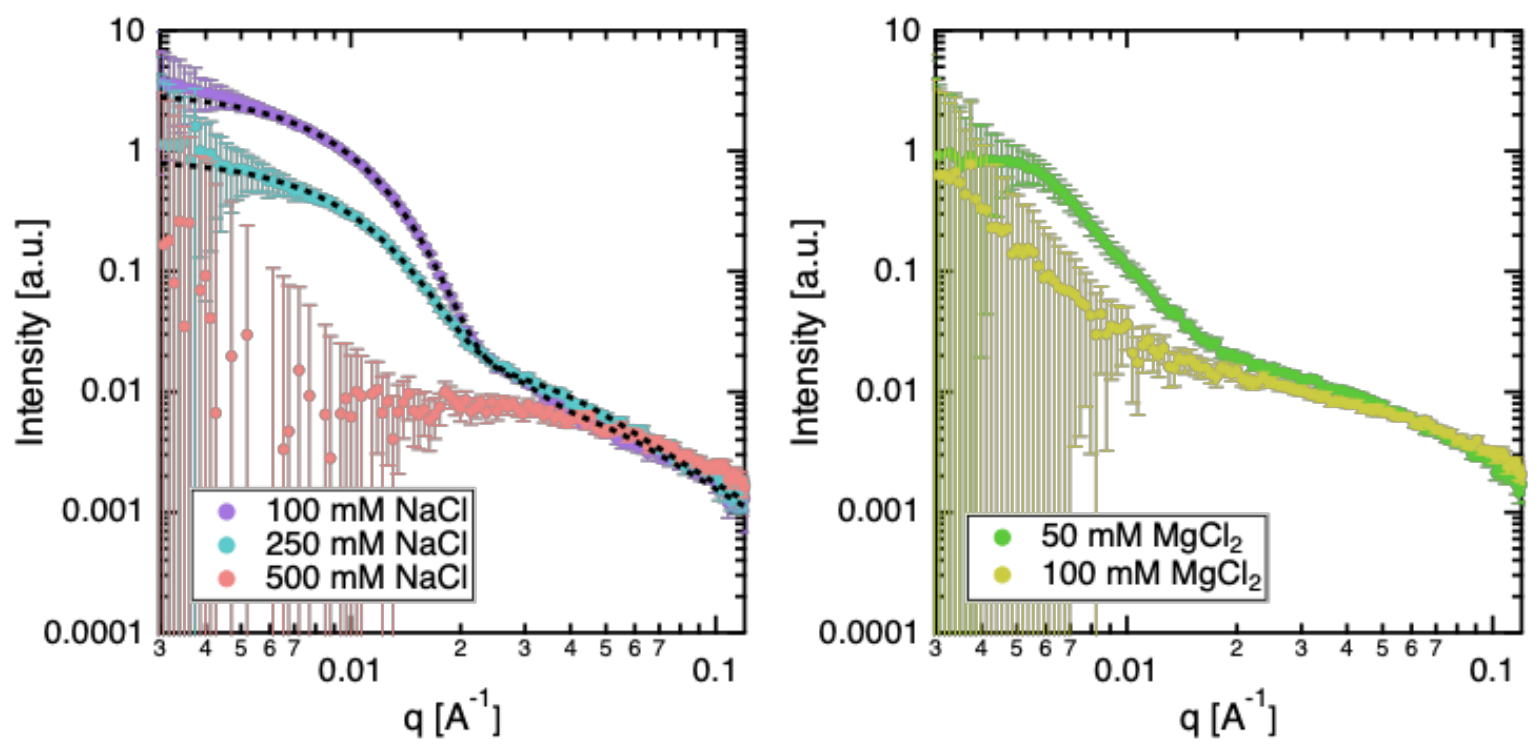

Figure S-42. SAXS profiles for $\mathrm{PMPC}_{5 \mathrm{~K}}-\mathrm{PVBTMA}_{50} / \mathrm{PAA}_{50}$ with 100, 250, and $500 \mathrm{mM} \mathrm{NaCl}$ (left), as well as 50 and $100 \mathrm{mM} \mathrm{MgCl}_{2}$ (right).
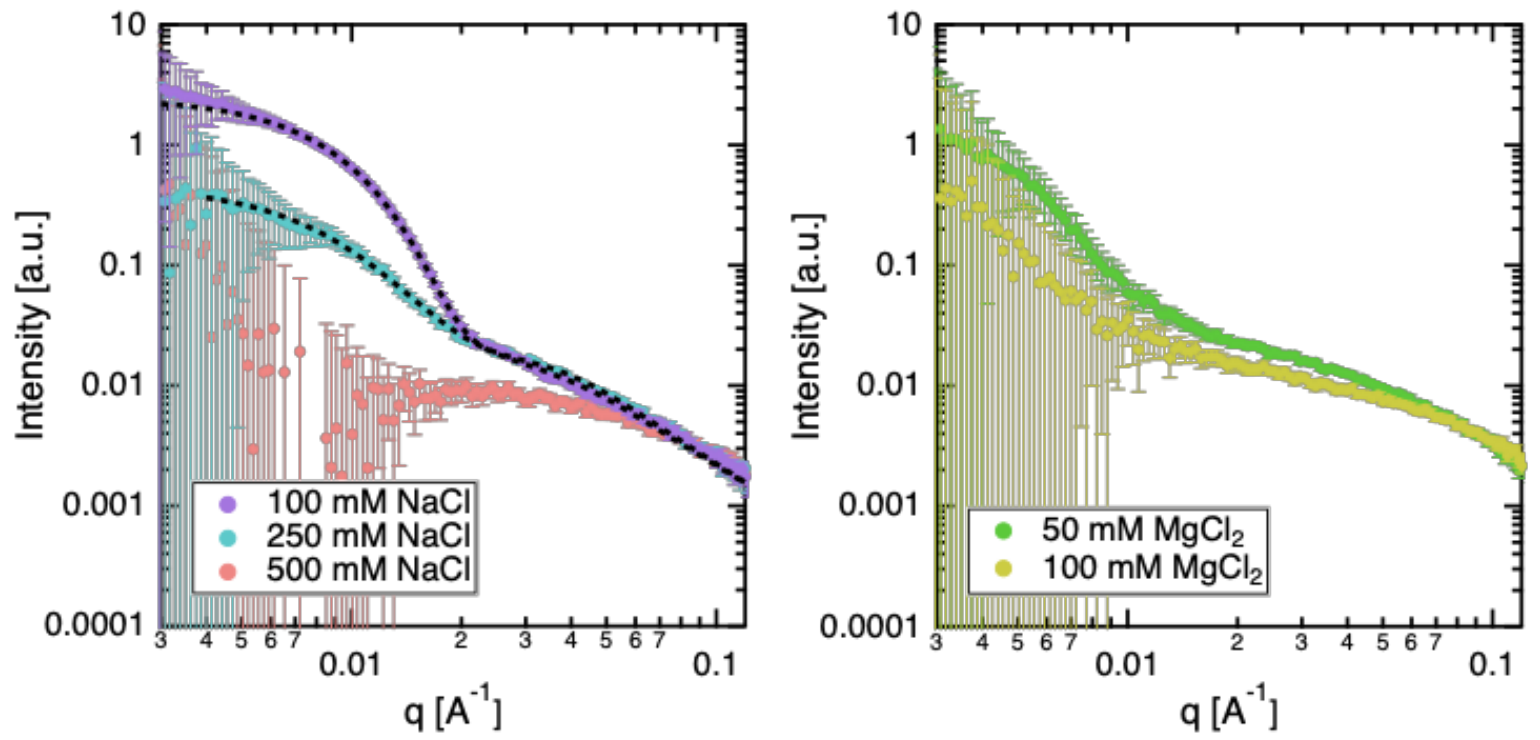

Figure S-43. SAXS profiles for $\mathrm{PMPC}_{10 \mathrm{~K}}-\mathrm{PVBTMA}_{97} / \mathrm{PAA}_{50}$ with 100, 250, and $500 \mathrm{mM} \mathrm{NaCl}$ (left), as well as 50 and $100 \mathrm{mM} \mathrm{MgCl}_{2}$ (right). 

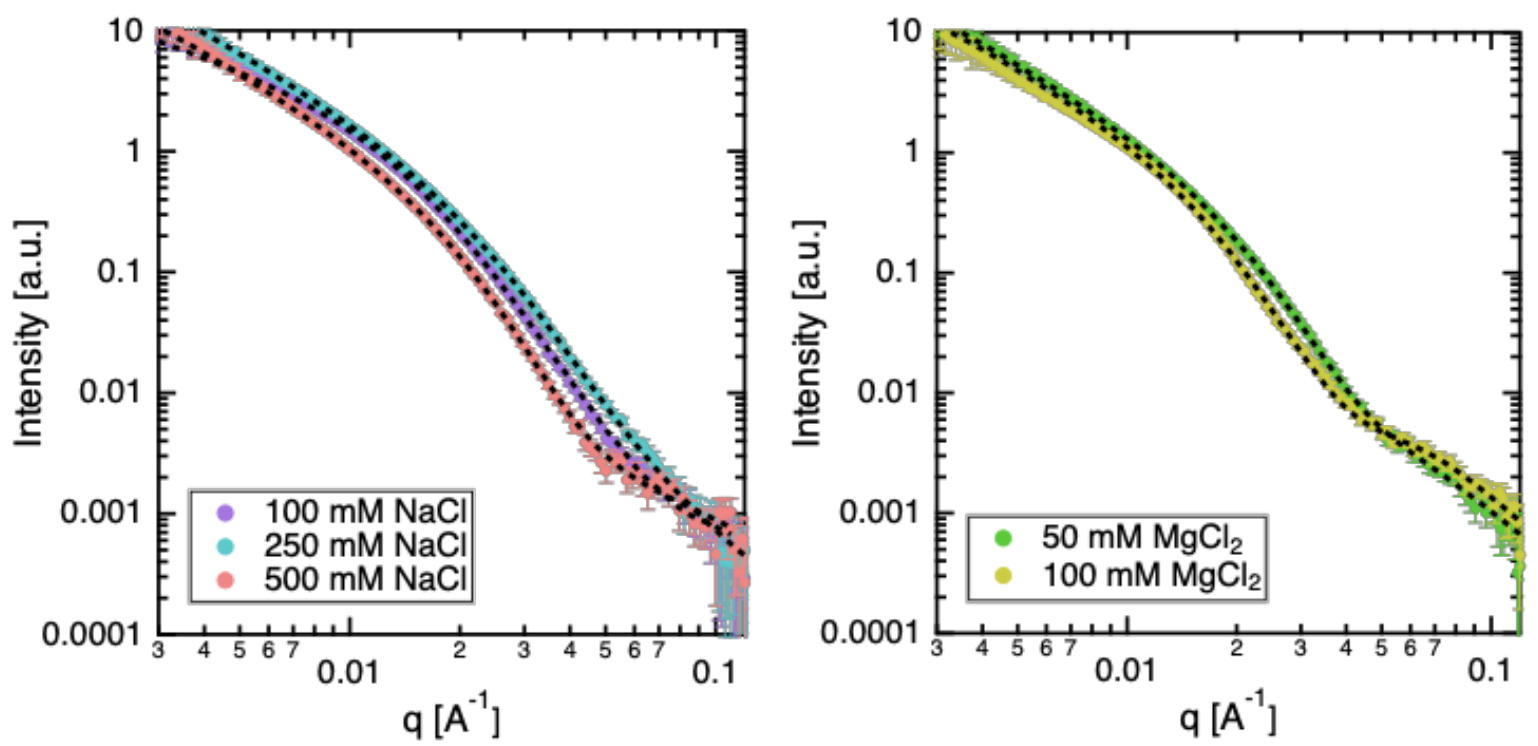

Figure S-44. SAXS profiles for $\mathrm{PEG}_{5 \mathrm{~K}}-\mathrm{PLK}_{47} / \mathrm{PAA}_{50}$ with 100,250 , and $500 \mathrm{mM} \mathrm{NaCl}$ (left), as well as 50 and $100 \mathrm{mM} \mathrm{MgCl}_{2}$ (right).
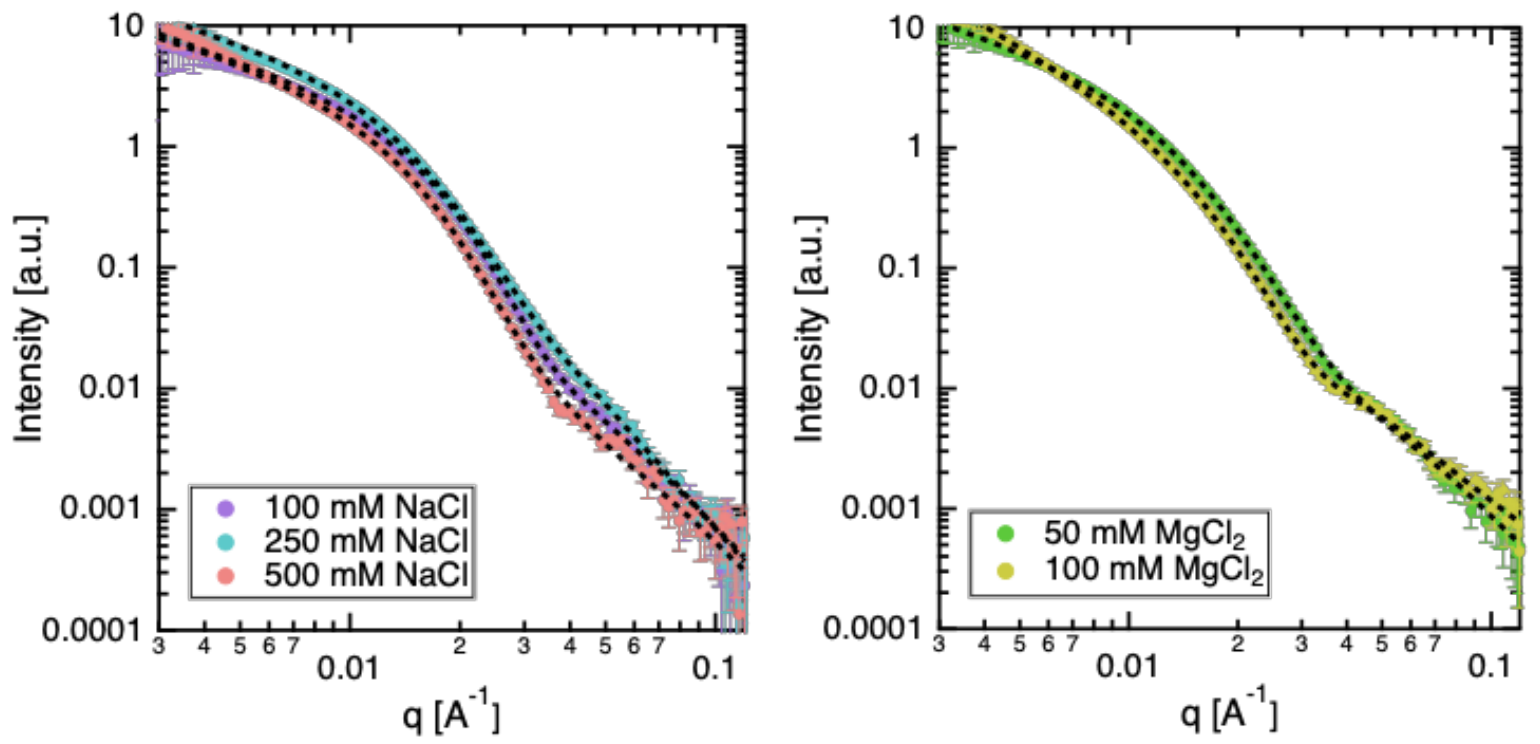

Figure S-45. SAXS profiles for $\mathrm{PEG}_{10 \mathrm{~K}}-\mathrm{PLK}_{93} / \mathrm{PAA}_{50}$ with 100, 250, and $500 \mathrm{mM} \mathrm{NaCl}$ (left), as well as 50 and $100 \mathrm{mM} \mathrm{MgCl}_{2}$ (right). 


\section{S4. Supplemental Polyelectrolyte Complex Micelle Stability Data}

Kinetic stability tests of the micelles were examined in FBS. Figure S-46 shows the apparent size distribution of PEG-PVBTMA and PMPC-PVBTMA at 1 and $10 \mathrm{~h}$, repeated three times independently. The overall size distribution of particles appears to be consistent, with evidence of PMPC-PVBTMA micelle aggregation over time.

A)

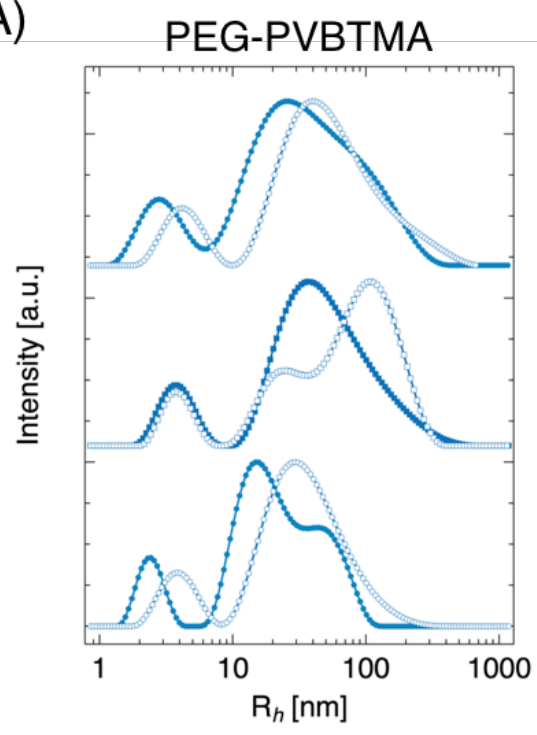

B)

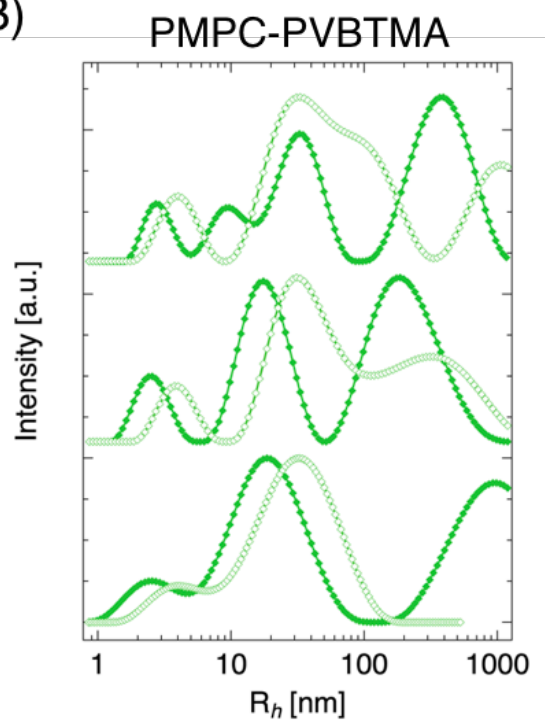

Figure S-46. Three replicates of the apparent size hydrodynamic radius distribution of PEGPVBTMA and PMPC-PVBTMA micelles at $1 \mathrm{~h}$ (solid markers) and $10 \mathrm{~h}$ (open markers), analyzed by REPES analysis at the $90^{\circ}$ angle. Intensity is scaled for clarity. 
